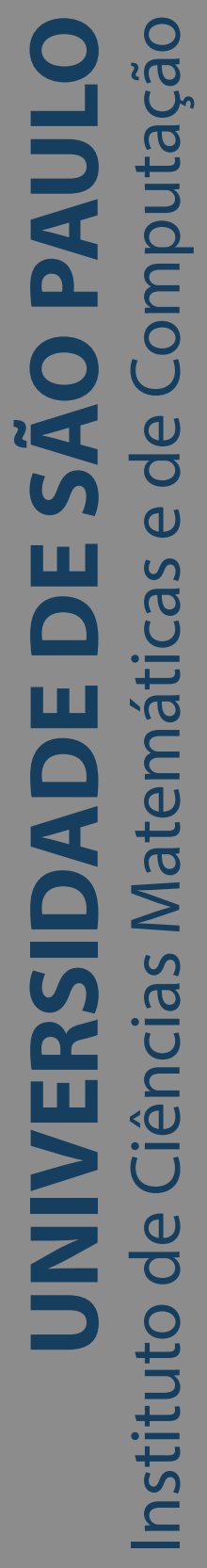

\title{
Cross-project defect prediction with meta-learning
}

\section{Faimison Rodrigues Porto}

Tese de Doutorado do Programa de Pós-Graduação em Ciências de Computação e Matemática Computacional (PPG-CCMC) 

SERVIÇO DE PÓS-GRADUAÇÃO DO ICMC-USP

Data de Depósito:

Assinatura:

\section{Faimison Rodrigues Porto}

\section{Cross-project defect prediction with meta-learning}

Doctoral dissertation submitted to the Institute of Mathematics and Computer Sciences - ICMC-USP, in partial fulfillment of the requirements for the degree of the Doctorate Program in Computer Science and Computational Mathematics. FINAL VERSION

Concentration Area: Computer Science and Computational Mathematics

Advisor: Prof. Dr. Adenilso da Silva Simão 
Ficha catalográfica elaborada pela Biblioteca Prof. Achille Bassi e Seção Técnica de Informática, ICMC/USP, com os dados fornecidos pelo(a) autor(a)

Rodrigues Porto, Faimison
Cross-project defect prediction with meta-
learning / Faimison Rodrigues Porto; orientador
Adenilso da Silva Simão. -- São Carlos, 2017.
124 p.
Tese (Doutorado - Programa de Pós-Graduação em
Ciências de Computaço e Matemática Computacional) --
Instituto de Ciências Matemáticas e de Computação,
Universidade de São Paulo, 2017.
1. software quality assurance. 2. software
defect prediction. 3. cross-project defect
prediction. 4. experimental software engineering.
5. meta-learning. I. da Silva Simão, Adenilso,
orient. II. Título.




\title{
Faimison Rodrigues Porto
}

\section{Predição de defeitos cruzada entre projetos apoiada por meta-aprendizado}

\author{
Tese apresentada ao Instituto de Ciências \\ Matemáticas e de Computação - ICMC-USP, \\ como parte dos requisitos para obtenção do título \\ de Doutor em Ciências - Ciências de Computação e \\ Matemática Computacional. VERSÃO REVISADA \\ Área de Concentração: Ciências de Computação e \\ Matemática Computacional \\ Orientador: Prof. Dr. Adenilso da Silva Simão
}

USP - São Carlos

Novembro de 2017 

To all the people who don't know what a PhD is. To all the people who believe in better days but depends on their governments. To all the people who are ready to change the world but don't know how to start. 

When I start thinking about the acknowledgments, it comes to my mind not only names but expressions. I remember people who had no word to say but with the eyes told me "I believe in you". And I am very grateful to say that those eyes did not come only from close friends or family. Is there something beyond our knowledge that unifies our lives? I believe so. So first, I would like to thank this whole thing that some call God, Nature, Buddha, Energy...

My family is my safe haven. All my professional and human development is done with them in mind. Thank you, my father, for all your lessons about responsibility. I will not run from mine. Thank you, my mother, for all your lessons about loving your neighbor, not being attached to material things, and the art of taking risks. My beloved sister, thank you for all your support and kindness. My brother, you rock... you inspire me to cross the line whenever necessary.

Some would say that "friend is that special one person whom you can trust". I disagree. Life is a flowing river. I prefer to see my friends as everyone whom I have a kind feeling, even the ones far from me or the ones no longer present in my life. I like to think that I am a big mosaic composed of little pieces originated from every experience and person that eventually became part of my history. So, to all the little pieces, old and new friends... Thank you. I am what I am because of you.

I would like to thank all the great leaders. Those people with reduced ego capable of perceiving a less illusory reality. Those people with a sense of fairness and respect for others. Those people who have "grow" and "give" as strong values. I knew some in my life. But here, specifically, I would like to thank my advisor Adenilso Simao who have taught me with his example more than 1000 words could do.

Thank you, Emilia Mendes and Leandro Minku, for your valuable advice during my abroad staying.

This work, however, would not be possible without the help of some friends who shared with me their valuable knowledge and support: Mantovas, Davi, and Dió. My trajectory is full of great people who contributed somehow for this moment: Carol, Alhine, FCarlos, Sid, Frota, Cabeça, Endo, Durelli, Jorge, Mauricio, Rachel, Geo, Fefa, Valéria, Silvana, Pedro, Leo, Raul, Joice, Draylson, Lina, Danillo, Vânia, Venilton, Rodrigo Calvo, Bruno Feres, Lucas Vendramin, Danilo Horta, Volpato, Valdemas, Iohan, Alfredo, André 
Zé, Odair, Paulão, Jaum, Lívia, Flávio, Damas, Kamila, Lilian, Rodrigo Magalhães, Vinícius Barbosa, Jefferson, Tiago João, Fabiana, Mariana, Katri, André Felipe, Murilo Otário, Hugo Sica, Zé Marquez, Gustavo Lourenço, Claudia Fabiano, Roberto Finzi, Liliane Vale, Leandro Souza, Claudio Costa, Fabiano Cunha, Vinícius Mello, Bleno, Douglas Rodovalho, Roberta Elias, Wilson, Joelma Burin. For sure I will forget some names... but the mosaic will not.

For the great philosophic moments, thank you Dario, Brauner, Nilton, Dió, Wil, Elias, Steve, Daniel, Fausto.

To my old friends who the destiny brought us to the same place to share silly and great moments. Thank you Adam, Lucas, Rayner, Fefo, Ed.

To my old friends of República Coqueiro. You will always be the golden part of my life. Thank you Pezão, Ursão, Negão, Erik, Bené.

I would like to acknowledge the support granted by FAPESP (grant 2013/01084-3). To all the teachers and employees of the ICMC, your work is very important for us all. 
We are here in the world to do nothing but share. 



\section{RESUMO}

PORTO, F. R. Predição de defeitos cruzada entre projetos apoiada por metaaprendizado. 2017. 124 p. Tese (Doutorado em Ciências - Ciências de Computação e Matemática Computacional) - Instituto de Ciências Matemáticas e de Computação, Universidade de São Paulo, São Carlos - SP, 2017.

Modelos de predição de defeitos auxiliam profissionais de teste na priorização de partes do software mais propensas a conter defeitos. A abordagem de predição de defeitos cruzada entre projetos (CPDP) refere-se à utilização de projetos externos já conhecidos para compor o conjunto de treinamento. Essa abordagem é útil quando a quantidade de dados históricos de defeitos é inapropriada ou insuficiente para compor o conjunto de treinamento.

Embora o princípio seja atrativo, o desempenho de predição é um fator limitante nessa abordagem. Nos últimos anos, vários métodos foram propostos com o intuito de melhorar o desempenho de predição de modelos CPDP. Contudo, na literatura, existe uma carência de estudos comparativos que apontam quais métodos CPDP apresentam melhores desempenhos. Além disso, não há evidências sobre quais métodos CPDP apresentam melhor desempenho para um domínio de aplicação específico.

De fato, não existe um algoritmo de aprendizado de máquina que seja apropriado para todos os domínios de aplicação. A tarefa de decisão sobre qual algoritmo é mais adequado a um determinado domínio de aplicação é investigado na literatura de meta-aprendizado. Um modelo de meta-aprendizado é caracterizado pela sua capacidade de aprender a partir de experiências anteriores e adaptar seu viés de indução dinamicamente de acordo com o domínio alvo. Neste trabalho, nós investigamos a viabilidade de usar meta-aprendizado para a recomendação de métodos CPDP.

Nesta tese são almejados três principais objetivos. Primeiro, é conduzida uma análise experimental para investigar a viabilidade de usar métodos de seleção de atributos como procedimento interno de dois métodos CPDP, com o intuito de melhorar o desempenho de predição. Segundo, são investigados quais métodos CPDP apresentam um melhor desempenho em um contexto geral. Nesse contexto, também é investigado se os métodos com melhor desempenho geral apresentam melhor desempenho para os mesmos conjuntos de dados (ou projetos de software). Os resultados revelam que os métodos CPDP mais adequados para um projeto podem variar de acordo com as características do projeto sendo predito. Essa constatação conduz à terceira investigação realizada neste trabalho. Foram investigadas as várias particularidades inerentes ao contexto CPDP a fim de propor uma solução de meta-aprendizado capaz de aprender com experiências anteriores e recomendar métodos CPDP adequados, de acordo com as características do software. Foram avaliados a capacidade de meta-aprendizado da solução proposta e a sua performance em relação 
aos métodos base que apresentaram melhor desempenho geral.

Palavras-chave: qualidade de software, predição de defeitos em software, predição de defeitos cruzada entre projetos, engenharia de software experimental, meta-aprendizado . 


\section{ABSTRACT}

PORTO, F. R. Cross-project defect prediction with meta-learning. 2017. 124 p. Tese (Doutorado em Ciências - Ciências de Computação e Matemática Computacional) - Instituto de Ciências Matemáticas e de Computação, Universidade de São Paulo, São Carlos - SP, 2017.

Defect prediction models assist tester practitioners on prioritizing the most defect-prone parts of the software. The approach called Cross-Project Defect Prediction (CPDP) refers to the use of known external projects to compose the training set. This approach is useful when the amount of historical defect data of a company to compose the training set is inappropriate or insufficient.

Although the principle is attractive, the predictive performance is a limiting factor. In recent years, several methods were proposed aiming at improving the predictive performance of CPDP models. However, to the best of our knowledge, there is no evidence of which CPDP methods typically perform best. Moreover, there is no evidence on which CPDP methods perform better for a specific application domain.

In fact, there is no machine learning algorithm suitable for all domains. The decision task of selecting an appropriate algorithm for a given application domain is investigated in the meta-learning literature. A meta-learning model is characterized by its capacity of learning from previous experiences and adapting its inductive bias dynamically according to the target domain. In this work, we investigate the feasibility of using meta-learning for the recommendation of CPDP methods.

In this thesis, three main goals were pursued. First, we provide an experimental analysis to investigate the feasibility of using Feature Selection (FS) methods as an internal procedure to improve the performance of two specific CPDP methods. Second, we investigate which CPDP methods present typically best performances. We also investigate whether the typically best methods perform best for the same project datasets. The results reveal that the most suitable CPDP method for a project can vary according to the project characteristics, which leads to the third investigation of this work. We investigate the several particularities inherent to the CPDP context and propose a meta-learning solution able to learn from previous experiences and recommend a suitable CDPD method according to the characteristics of the project being predicted. We evaluate the learning capacity of the proposed solution and its performance in relation to the typically best CPDP methods.

Keywords: software quality assurance, software defect prediction, cross-project defect prediction, experimental software engineering, meta-learning . 

Figure 1 - General process to construct an SDP model (Adapted from (NAM, 2015)). 38

Figure 2 - Summary of the SDP literature along the last 50 years (Adapted from $(\mathrm{NAM}, 2015)) \ldots \ldots \ldots \ldots \ldots \ldots$

Figure 3 - Example of an SVM applied to a set of linearly separable data. The highlighted instances represent the Support Vectors, the center line represents the hyperplane and the dashed lines represent the margins. . 43

Figure 4 - Example of an MLP network. . . . . . . . . . . . . . . 44

Figure 5 - Example of a C4.5 tree model generated for 12 hypothetical instances. The target attribute contains two possible labels (Defective or NonDefective). . . . . . . . . . . . . . . . . . . . 45

Figure 6 - Example of a Random Forest model. . . . . . . . . . . . . . . . . 46

Figure 7 - Different learning process between traditional machine learning and transfer learning (Adapted from (PAN; YANG, 2010)). . . . . . . . . . 47

Figure 8 - General process of a meta-learning system for algorithm recommendation (Adapted from (SERBAN et al., 2013)).

Figure 9 - Illustration of the procedure executed for each filtering method evaluated. On Figure (a), the colored instances represent the cross-project training data and the white instances represent the test data. See text for more details (Adapted from (PETERS; MENZIES; MARCUS, 2013)) . . . . .

Figure 10 - Pseudocode with the experiment design for both Classification and Ranking. . . . . . . . . . . . . . . . . . . . . 64

Figure 11 - Ratio of successful models for practical use considering the thresholds

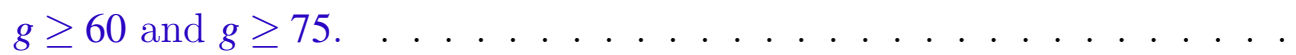

Figure 12 - All 31 evaluated CPDP methods derived from the combination of six transfer learning solutions and five classifiers. . . . . . . . . . . . .

Figure 13 - Regions of a typical ROC curve (MENZIES; GREENWALD; FRANK, 2007) . . . . . . . . . . . . . . . . .

Figure 14 - Pseudocode of the experiment design with Cross-Project Leave One Out (CPLOO) cross-validation. . . . . . . . . . . . . . . 8

Figure 15 - Pairwise Post-hoc Fisher's LSD test. Each letter represents a group of methods with no statistical significant difference. 
Figure 16 - Data of Table 16 organized by intersection sets. Each CPDP method represents a set of all projects in which it achieved the best performance. The bars represent the distribution of all 47 projects along the four methods. When a project has more than one best method, the intersection is represented by the connected dots. . . . . . . . . . . . 88

Figure 17 - Proposed architecture: meta-learning for CPDP . . . . . . . . . . . . . 94

Figure 18 - The resulting best accuracies generated for each meta-model in the feature selection step. The majority class is used as a comparative reference for the meta-features sets MS-Dist and MS-Uns. . . . . . . . 101

Figure 19 - Pairwise Post-hoc Fisher's LSD test. The meta-learner MS-Dist presents the best ranking position along with the base method 2012Ma_nb, with no significant difference. . . . . . . . . . . . . . . . . . . . 103 
Table 1 - Software quality attributes suggested in (BOEHM; BROWN; KASPAR,

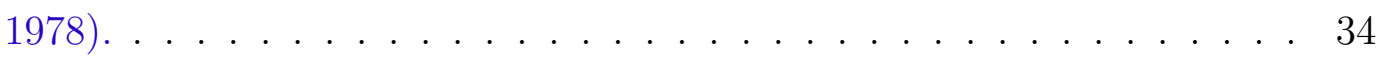

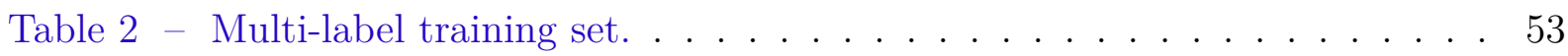

Table 3 - Summary of the projects characteristics for Ranking. We analyzed a ranking performance measure that measures the percentage of defects $(\alpha)$ in the former $(\beta)$ instances of the ranking. Usually, in the literature, $\beta=20 \%$. In this study we fixed $\alpha \approx 80 \%$ and the $\beta$ varies depending on the defect distribution for each project. . . . . . . . . . . 60

Table 4 - Summary of the projects characteristics for Classification. . . . . . . . 61

Table 5 - Code metrics (Descriptions adapted from (JURECZKO; MADEYSKI, 2010)). . . . . . . . . . . . . . . . .

Table 6 - The analyzed network metrics. The metrics were computed for incoming (In), outgoing (Out), and/or undirected (All) dependencies (Descriptions adapted from (ZIMMERMANN; NAGAPPAN, 2008) and (HERZIG et al., 2013)). . . . . . . . . . . . . . . .

Table 7 - Performance measures for Classification. . . . . . . . . . . .

Table 8 - Performance obtained for the analyzed IFFS methods for both Burak and Peters Filters for Classification. All the performances are presented in relation to noFilter. Positive values mean better performances and negative values mean lower performances. The bold values indicate the highest performance for a project row. The symbols ${ }^{\oplus}$ and ${ }^{\ominus}$ represent the columns with significant difference in relation to noFilter. . . . . . . 68

Table 9 - Performance comparison of the $\alpha$ percentage of defects in the top $\beta$ instances of Ranking, obtained for each evaluated method. The performances (except Random) are presented in relation to noFilter. Positive values mean better performances and negative values mean lower performances. The bold values indicate the highest performance for a project row. The symbols ${ }^{\oplus}$ and ${ }^{\ominus}$ represent the columns with significant difference in relation to noFilter. . . . . . . . . . . . . . . . . . . . 70 
Table 10 - Performance comparison based on the ranking measure FPA. The columns Max and Random are comparative references. All other performances are presented in relation to noFilter. Positive values mean better performances and negative values mean lower performances. The symbols ${ }^{\oplus}$ and ${ }^{\ominus}$ represent the columns with significant difference in relation to noFilter. . . . . . . . . . . . . . . . . 71

Table 11 - Frequency in which a IFFS method performs among the top five prediction performances for a project (Classification). . . . . . . . . . . . 72

Table 12 - Frequency in which a IFFS method performs among the top five prediction performances for a project (Ranking). . . . . . . . . . . . . 72

Table 13 - Summary of the projects characteristics. . . . . . . . . . . . . 79

Table 14 - Performance obtained for each of the 31 CPDP methods considering the mean (and standard deviation) values for all 47 software projects. . . . . 86

Table 15 - Comparison of CPDP methods in relation to the baseline original learners, based on the statistical Fisher's LSD test. The symbol "/" means no statistical difference. The symbols " $(+)$ " or "(-)" mean statistical difference with better or worse mean rank, respectively. . . . . . . . . 87

Table 16 - Best AUC value and respective best CPDP methods by project, considering the four methods with better ranking across datasets. The AUC values were rounded to 2 significant digits in order to not differentiate similar performances. . . . . . . . . . . . . . . . . . . . . 8 89

Table 17 - Unsupervised meta-features set (MS-Uns), where $\mathscr{U}$ represents a dataset with $n$ instances, $x \in \mathscr{U}$ represents an instance of $\mathscr{U}$, and $1<j<20$ represents a feature of $\mathscr{U}$ (Adapted from (SANTOS; CARVALHO, 2016)). 97

Table 18 - The estimated accuracies and selected subsets for each meta-model. The majority class is used as a comparative reference. . . . . . . . . . . . 101

Table 19 - The general performance of the proposed meta-learning solution for both meta-features sets. These performances are compared with the four base CPDP methods and the random baseline. . . . . . . . . . . . . . . . 102

Table 20 - Part I - General AUC performances for all the evaluated CPDP methods (Base and Meta). The methods are ordered by the mean AUC value.

Table 21 - Part II - General AUC performances for all the evaluated CPDP methods (Base and Meta). The methods are ordered by the mean AUC value. . 124 


\section{LIST OF ABBREVIATIONS AND ACRONYMS}

\begin{tabular}{|c|c|}
\hline $\mathrm{AMC}$ & Average Method Complexity \\
\hline AUC & Area Under the Receiver Operating Characteristic Curve \\
\hline $\mathrm{BR}$ & Binary Relevance \\
\hline C45 & C4.5 \\
\hline $\mathrm{Ca}$ & Afferent couplings \\
\hline CAM & Cohesion Among Class Methods \\
\hline $\mathrm{CBM}$ & Coupling Between Methods \\
\hline $\mathrm{CBO}$ & Coupling between object classes \\
\hline $\mathrm{CC}$ & Cyclomatic Complexity \\
\hline $\mathrm{Ce}$ & Efferent couplings \\
\hline CODE & code metrics \\
\hline CPD & Cross-Project Dataset \\
\hline CPDP & Cross-Project Defect Prediction \\
\hline CPLOO & Cross-Project Leave-One-Out \\
\hline DAM & Data Access Metric \\
\hline DIT & Depth of Inheritance Tree \\
\hline $\mathrm{FN}$ & False Negative \\
\hline $\mathrm{FP}$ & False Positive \\
\hline FPA & Fault-Percentile-Average \\
\hline FS & Feature Selection \\
\hline $\mathrm{IC}$ & Inheritance Coupling \\
\hline IFFS & Instance Filtering methods based on Feature subset Selection \\
\hline JIT & Just-In-Time \\
\hline $\mathrm{KNN}$ & K-Nearest Neighbor \\
\hline LCOM & Lack of cohesion in methods \\
\hline LCOM3 & Lack of cohesion in methods \\
\hline LOC & Lines of Code \\
\hline MFA & Measure of Functional Abstraction \\
\hline MLP & Multilayer Perceptron \\
\hline MOA & Measure of Aggregation \\
\hline MS-Dist & Distributional Meta-features Set \\
\hline
\end{tabular}


MS-Uns Unsupervised Meta-features Set

NB Naïve Bayes

NET network metrics

NOC Number of Children

NPM Number of Public Methods

OO Object-Oriented

PD Probability of Detection

PF Probability of False Alarm

RF Random Forest

RFC Response for a Class

ROC Receiver Operating Characteristic

SDP Software Defect Prediction

SS Software metric Subsets

SUT System Under Test

SVM Support Vector Machine

TCA Transfer Component Analysis

TN True Negative

TP True Positive

WMC Weighted methods per class

WPDP Within-Project Defect Prediction 


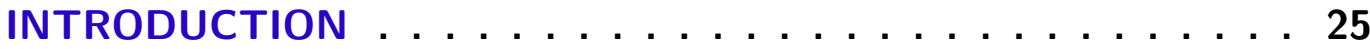

$1.1 \quad$ Context and Motivation . . . . . . . . . . . . . 25

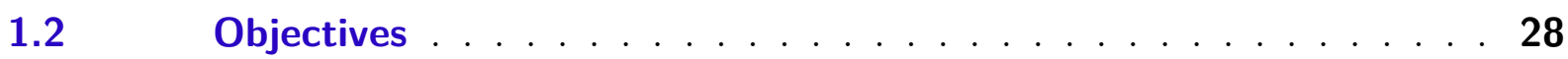

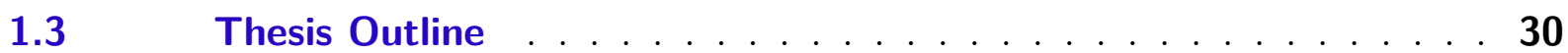

2 BACKGROUND AND RELATED WORK . . . . . . . . . . . 33

$2.1 \quad$ Software Testing . . . . . . . . . . . . . . 33

2.2 Software Defect Prediction . . . . . . . . . . . . . . . 38

2.3 Machine Learning Algorithms for Software Defect Prediction . . . . 42

2.3.1 Support Vector Machines . . . . . . . . . . . . . . . . . . . 42

2.3.2 Multilayer Perceptron . . . . . . . . . . . . . . . . . . . . 43

2.3.3 Naïve Bayes . . . . . . . . . . . . . . . . . . . . 44

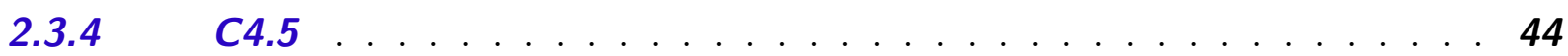

2.3.5 Random Forest . . . . . . . . . . . . . . . . . . . . 45

$2.4 \quad$ Cross-project Defect Prediction . . . . . . . . . . . . . . . . 45

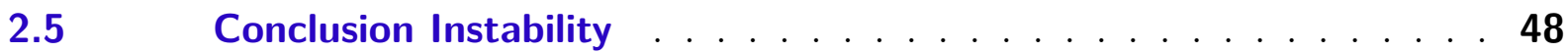

2.6 Meta-learning for Algorithm Recommendation . . . . . . . . . . 50

2.7 Multi-label Learning . . . . . . . . . . . . . . . . . 52

$2.8 \quad$ Summary . . . . . . . . . . . . . . . . . . . 54

3 FEATURE SUBSET SELECTION AND INSTANCE FILTERING FOR CPDP - CLASSIFICATION AND RANKING . . . . . . . . . 55

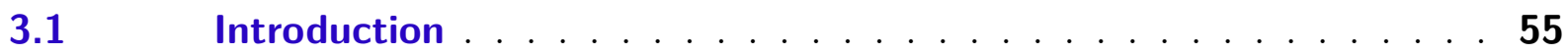

$3.2 \quad$ Instance Filtering Methods . . . . . . . . . . . . . . 57

$3.3 \quad$ Feature Subset Selection . . . . . . . . . . . . . . . . . . 57

$3.4 \quad$ Experimental Setup . . . . . . . . . . . . . . . . 58

3.4.1 Data Collection . . . . . . . . . . . . . . . . . . . . 59

3.4.1.1 Code Metrics . . . . . . . . . . . . . . . . . . . . . . . . 59

3.4.1.2 Network Metrics . . . . . . . . . . . . . . . . . . . 59

3.4.2 Feature Subset Selection . . . . . . . . . . . . . . . . . .61

3.4.3 Experiment Design . . . . . . . . . . . . . . . . . . . . 64

3.4.4 Prediction Models . . . . . . . . . . . . . . . . 65

3.4.5 Performance Measures . . . . . . . . . . . . . . . . . . . 65 
$3.5 \quad$ Results . . . . . . . . . . . . . . . . 67

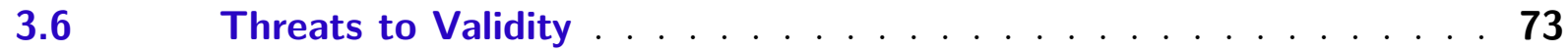

$3.7 \quad$ Conclusion . . . . . . . . . . . . . . . . . . . . . 74

$4 \quad$ PERFORMANCE EVALUATION OF CPDP METHODS $\ldots \ldots 77$

$4.1 \quad$ Introduction . . . . . . . . . . . . . . . . . . . 77

$4.2 \quad$ Experimental Setup . . . . . . . . . . . . . . . . 78

4.2.1 Software Projects . . . . . . . . . . . . . . . . . . . . 78

4.2.2 Data Preprocessing . . . . . . . . . . . . . . . . . . . 80

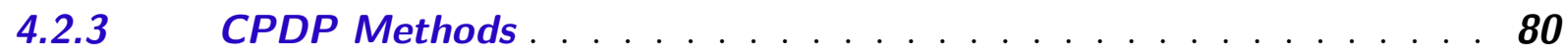

4.2.4 Performance Measure . . . . . . . . . . . . . . . . . . . 82

4.2.5 Experiment Design . . . . . . . . . . . . . . . . . . . . . . 84

4.2.6 Statistical Test . . . . . . . . . . . . . . . . . . . . . . . . 84

$4.3 \quad$ Results . . . . . . . . . . . . . . . . . . . . 85

$4.4 \quad$ Threats to Validity . . . . . . . . . . . . . . . . . 90

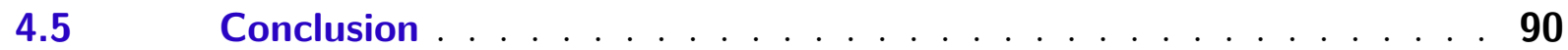

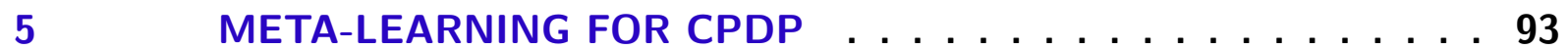

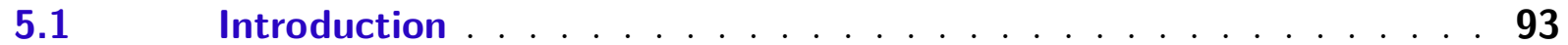

$5.2 \quad$ Proposed Methodology . . . . . . . . . . . . . . . . . . 94

5.2.1 Input Datasets . . . . . . . . . . . . . . . . . . 95

5.2.2 Meta-features . . . . . . . . . . . . . . . . . 95

5.2.3 Meta-target . . . . . . . . . . . . . . . . 96

5.2.4 Meta-data Preprocessing . . . . . . . . . . . . 97

5.2.5 Meta-learner . . . . . . . . . . . . . . . . . . . . 98

5.2.6 Performance Measure . . . . . . . . . . . . . . . . . 98

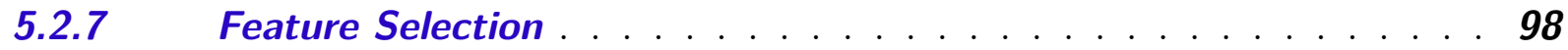

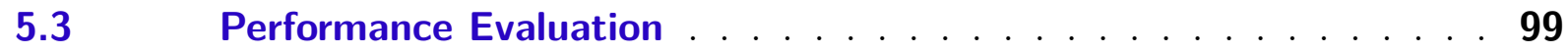

5.3.1 Meta-level . . . . . . . . . . . . . . . . . . 100

5.3.2 Base-level . . . . . . . . . . . . . . . . . . . 100

$5.4 \quad$ Results . . . . . . . . . . . . . . . . . . . . . . 100

$5.5 \quad$ Threats to Validity . . . . . . . . . . . . . . . . . . . 103

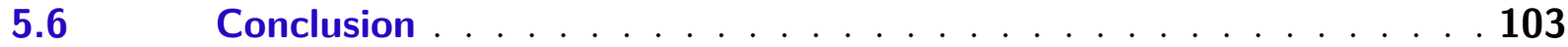

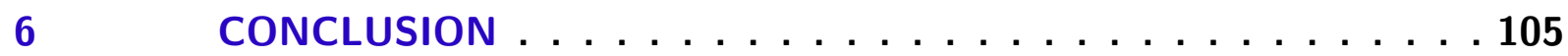

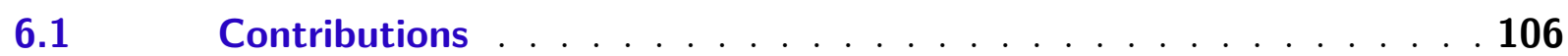

6.2 Limitations and Future Work . . . . . . . . . . . . . . . . . 107 

CHAPTER

1

INTRODUCTION

\subsection{Context and Motivation}

The size and complexity of software systems have grown gradually over time. In parallel, the demand for efficient procedures able to reduce cost and increase the quality of the final software product also increases. In this context, the testing activities are designed to verify the correct operation of the system through its execution. These activities perform an important role in the development process since they represent the final revision of the software specification, project and codification (PRESSMAN, 2009). The application of testing allows the early identification of defects. The earlier a defect is identified in the development process, the lower is the cost for its maintenance (BOEHM; BASILI, 2001).

However, software testing is expensive (JONES; BONSIGNOUR, 2011). It usually represents the largest portion of the total cost and elapsed time spent in the development of a typical software project (MYERS; SANDLER; BADGETT, 2011). Therefore, in a scenario with limited resources (e.g., financial, time, manpower), a proper management of the available resources is necessary. A defect prediction model can be used in this context since it is designed to predict the most defect-prone parts of a software. For example, in a scenario where only $30 \%$ of testing resources are available, this knowledge can help the practitioners on prioritizing the most critical classes/modules of the software, hence, resulting in a lower cost, higher quality and more maintainable software in the given time and budget.

The success of a Software Defect Prediction (SDP) model depends on two main factors: 1) building an accurate training set; and 2) applying a suitable machine learning algorithm. In a classification task, the training set can be represented by a table of elements. In this table, each row represents an instance (software part or module); the independent variables (or features) represent the characteristics of each instance; and the dependent 
variable (class or target attribute) represents the binary class - defective or non-defective. Much effort has been spent in order to find effective independent variables, i.e., software properties able to provide relevant information for the learning task (OSTRAND; WEYUKER; BELL, 2004; ZIMMERMANN; NAGAPPAN, 2008; PINZGER; NAGAPPAN; MURPHY, 2008; HERZIG et al., 2013). Other studies are focused on the performance of machine learning algorithms applied to defect prediction (LESSMANN et al., 2008; GHOTRA; MCINTOSH; HASSAN, 2015) or on the impact of SDP models in industry (ENGSTRÖM; RUNESON; WIKSTRAND, 2010; BELL; WEYUKER; OSTRAND, 2011).

Commonly, in the literature, the defect prediction models are investigated in a within-project context which assumes the existence of previous defect data for a project (HERBOLD; TRAUTSCH; GRABOWSKI, 2016). In this context, the training set is obtained from a similar project or from previous releases of the same software. This approach is called Within-Project Defect Prediction (WPDP). However, in practice, many companies lack local defect data repositories (KITCHENHAM; MENDES; TRAVASSOS, 2007). This is due to two main reasons: 1) there are not enough previous data (e.g., the project is in its first release); or 2) the data were not properly collected due to the cost involved to maintain such data repositories (ZIMMERMANN et al., 2009; TURHAN et al., 2009). In these cases, an alternative approach is to assemble a training set composed of known external projects. Such data can be collected either from finished known projects or publically available data repositories. This approach, named Cross-Project Defect Prediction (CPDP), has attracted the interest of researchers in recent years due to its broader applicability (GUNARATHNA; TURHAN; HOSSEINI, 2016).

Although the principle is attractive, the predictive performance is a limiting factor. Zimmermann et al. (2009), for example, show in a large experiment that only $3.4 \%$ of 622 pairs of projects $\left(\right.$ project $_{1}$, project $_{2}$ ) presented adequate predictive power when project $_{1}$ was predicted based on a model trained with project $_{2}$. This CPDP approach is called one-to-one, where the modules of one unique project compose the training set. In another CPDP approach, called many-to-one, the modules of multiple projects compose the training set. In this latter approach, investigated in this thesis, two contrasting factors can be highlighted: 1) it provides a larger and diversified amount of data where the defect patterns can be learned, which is positive considering the low rate of compatible pairs of projects, as discussed in (ZIMMERMANN et al., 2009); and 2) it increases the heterogeneity of the data, which may decrease the efficiency of defect prediction models (TURHAN et al., 2009). In the literature, several solutions were proposed aiming at mitigating this heterogeneity and improve the learning capacity of CPDP models.

In the context of this thesis, we highlight the transfer learning solutions (PAN; YANG, 2010). In this approach, the solutions use some knowledge about the target domain in order to approximate the different distributions of source and target data. 
Different strategies are explored in this context, such as the transformation of data (WATANABE; KAIYA; KAIJIRI, 2008; CRUZ; OCHIMIZU, 2009), the filtering of relevant instances or projects (TURHAN et al., 2009; HERBOLD, 2013; HE et al., 2013), or the different weighting of relevant training instances (MA et al., 2012). However, to the best of our knowledge, no comprehensive comparison among these methods was conducted in the literature. Existing comparative analyzes are restricted to a few methods (MA et al., 2012; HE et al., 2013; HERBOLD; TRAUTSCH; GRABOWSKI, 2016) or do not provide a uniform experimental setup (GUNARATHNA; TURHAN; HOSSEINI, 2016). In addition, most of these methods can be associated to different machine learning algorithms, composing variations with different predictive performances. In this thesis, we investigate which methods are typically best in the context of CPDP. This knowledge can assist practitioners in choosing a method to use.

In this thesis, we also investigate the feasibility of using Feature Selection (FS) methods as an internal procedure of two CPDP methods aiming at improving their predictive performance. This study leads us to two major conclusions: first, the predictive performance of the two investigated CPDP methods can be improved when associated with feature selection methods; and second, the most suitable method for a project can vary according to the characteristics of the project being predicted. This latter statement is also investigated in this thesis for the general context of CPDP methods. We investigate whether the best CPDP methods perform better for the same groups of projects. This knowledge can accredit further strategies designed to assist practitioners in deciding which methods are more suitable for a given application domain.

In fact, each machine learning algorithm has an inductive bias (MITCHELL, 1997). This bias is a result of all the assumptions that an algorithm makes in order to generalize from the training data to unseen instances. Therefore, the inductive bias inherent for each algorithm makes it more (or less) suitable for a specific application domain. The no free lunch theorem, however, states that no inductive bias is suitable for all domains (WOLPERT, 2002). For an analyst, it requires a lot of experience to identify the most appropriate algorithm for the morphology of the problem at hand. Brodley and Smyth (1997) describe the process of selecting the appropriate models and algorithms for a specific problem.

Nevertheless, an analyst with the appropriate knowledge and experience is not always available. In addition, this process can be costly. Hence, automatic resources to recommend the most suitable solution for a problem are desirable. The automatic recommendation of algorithms is investigated as a sub-topic in the general meta-learning literature (LEMKE; BUDKA; GABRYS, 2015; BRAZDIL et al., 2017). A meta-learning model is characterized by its capacity of learning from previous experiences and adapt its inductive bias dynamically according to the target domain (BRAZDIL et al., 2017). In 
the context of this study, a meta-learning model should be able to recommend a suitable CPDP method according to the characteristics of the software being predicted.

In the literature, this task is approached as a typical classification problem, although at a meta-level (KALOUSIS, 2002; VILALTA; DRISSI, 2002; BRAZDIL; SOARES; COSTA, 2003; ALI; SMITH, 2006). In this context, the training meta-data is composed of: the meta-instances, representing the known previous experiences (or datasets); the meta-features, representing the dataset characteristics; and the meta-target, representing the more appropriate method for a specific dataset. The meta-learning task is to construct an inductive model in order to predict the classes of new unseen meta-instances.

Traditionally, a dataset is characterized using supervised meta-features, i.e., metafeatures extracted based on the target attribute (BRAZDIL; HENERY, 1994). Commonly, this traditional characterization is appropriate since each dataset (or meta-instance) represents the training set of a classification problem - designed to predict unseen instances from a similar domain. For example, the supervised characterization of datasets is appropriate for the recommendation of algorithms in the WPDP context (DORES et al., 2016). In the CPDP context, however, each training set is composed of heterogeneous data for the prediction of possibly non-similar unseen instances. In addition, considering the many-toone approach of CPDP, the same training set can be used to predict unseen instances of different projects. In other words, in the many-to-one CPDP approach, the information about which method performs better for a project can not be extracted from the source data but from the target data. These two statements make infeasible a meta-learning solution for CPDP based on the traditional supervised characterization of source data.

\subsection{Objectives}

Our general purpose in this thesis is to provide alternative solutions for CPDP and to provide support for test practitioners on selecting an appropriate CPDP method. More specifically, our main objective is to investigate the feasibility of using meta-learning for the recommendation of suitable CPDP methods according to the characteristics of the project being predicted. For this, we propose and evaluate an alternative meta-learning solution. In this solution, each meta-instance represents a target dataset ${ }^{1}$. Since the target dataset does not dispose of the target attribute information, we propose the characterization of meta-instances based on unsupervised meta-features. This and other particularities inherent to the CPDP context are carefully investigated and comprised in a general meta-learning architecture (KALOUSIS, 2002). The automatic recommendation of CPDP methods for a project can assist practitioners on selecting the most appropriate CPDP

1 Instead of the traditional characterization of source datasets, as already investigated in related work (DORES et al., 2016). 
model.

Part of the process to achieve this objective consists of evaluating the performance of the state-of-the-art CPDP methods and how they perform for specific datasets. For this, we conducted an extensive experimental comparison of 31 CPDP methods applied to 47 versions of 15 open source software projects. The 31 methods derived from the combination of six state-of-the-art transfer learning solutions with the five most frequently used classifiers in the defect prediction literature. We designed a uniform experimental setup to mitigate potential sources of bias that could accidentally lead to conclusion instability (MENZIES; SHEPPERD, 2012; SONG; MINKU; YAO, 2013; SHEPPERD; BOWES; HALL, 2014; TANTITHAMTHAVORN et al., 2016). From this experiment, we investigated which methods perform better across datasets. We managed to identify the four CPDP methods with general best performances. We also investigated how these four methods perform for different datasets. The results show that even though these four methods perform best across datasets and present similar performances to each other, they perform better for distinct groups of datasets. In other words, the most suitable method for a project can vary according to the project (or dataset) being predicted.

The objectives discussed above were initially motivated by an experimental analysis conducted by the authors and published in Porto and Simao (2016a) and Porto and Simao (2016b). In these studies we investigated the feasibility of using feature selection methods to improve the performance of two instance filtering methods for CPDP. We evaluated 41 different variations combining four distinct FS methods with the CPDP methods proposed by Turhan et al. (2009) and Peters, Menzies and Marcus (2013). We call the evaluated methods as Instance Filtering methods based on Feature subset Selection (IFFS). The results from this experiment indicate that the performance of the evaluated CPDP methods can be improved when combined with FS methods. However, the most appropriate CPDP method for a project can vary according to the project characteristics. This latter statement was also revealed in the general context of CPDP, as mentioned above. This statement accredited the investigation of the proposed meta-learning solution.

In this thesis, we aim at answering the following research questions:

RQ3.1 Can IFFS methods lead to better performances on CPDP models?

RQ3.2 Which IFFS methods present better performances on CPDP?

RQ4.1 Which CPDP methods perform better across datasets?

RQ4.2 Do the best CPDP methods perform better for the same datasets?

RQ5.1 To what extent can meta-learning help us to select the most suitable CPDP method for a given dataset? 
RQ5.1.1 Does the meta-learner learn?

RQ5.1.2 How does the meta-learner perform across datasets?

\subsection{Thesis Outline}

This thesis is organized as follows. In Chapter 2, we present the necessary background and the related work. In Section 2.1, we present the basic concepts and terminologies of software testing. In Section 2.2, we present the basic concepts and the research conducted in the SDP literature along the last 50 years. In Section 2.3, we present the five most popular machine learning algorithms in the SDP literature according to a recent systematic literature review. In Section 2.4, we contextualize the CPDP concept and present the related work. In Section 2.5, we discuss the factors that contribute to the conclusion instability, commonly present in the experimental software engineering research area. In Section 2.6, we present the basic concepts of meta-learning. In Section 2.7, we present the multi-label learning concepts. In Section 2.8, we summarize the main subjects discussed in the chapter.

In Chapter 3, we investigate the feasibility of applying feature selection methods to improve the performance of CPDP instance filtering methods. In Sections 3.2 and 3.3, we present the basic concepts of the proposed approach. In Section 3.4, we present the experimental setup for the conducted experiment. In Section 3.5, we discuss the obtained results. In Section 3.6, we present the threats to validity. In Section 3.7, we summarize the conducted experiment and the obtained results.

In Chapter 4, we present an extensive experimental comparison of 31 CPDP methods based on the state-of-the-art transfer learning solutions. In Section 4.2, we present the experimental setup, including the software data collection, the preprocessing resources, the evaluated CPDP methods, the performance measure, the statistical test and the experiment design. In Section 4.3, we evaluate and discuss the obtained results. In Section 4.4 we discuss the threats to validity inherent to the conducted experiment. In Section 4.5, we summarize the conducted experiment and the obtained results.

In Chapter 5, we propose and evaluate a meta-learning solution applied for CPDP. In Section 5.2, we propose a meta-learning architecture applied for the CPDP context. In this section, we present and discuss the specific particularities and internal issues involved in the proposed architecture, which includes the meta-data, the unsupervised meta-features, the preprocessing resources, the multi-label meta-target, the meta-learner, the performance measure, and the feature selection method. In Section 5.3, we define the experimental setup for the evaluation of the proposed solution in both levels: meta-level and base-level. In Section 5.4, we present and discuss the obtained results of performance for the proposed solution. In Section 5.5, we discuss the threats to validity. In Section 5.6, 
we summarize the proposed meta-learning solution and the obtained performance results.

In Chapter 6, we highlight the main contributions provided in this work and discuss the remaining open issues and possible ways to address them. 

CHAPTER

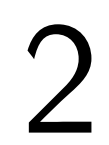

2

\section{BACKGROUND AND RELATED WORK}

In this chapter, we present the background concepts and the related work necessary for the understanding of the study conducted in the remaining of this thesis. In Section 2.1 we present the basic concepts and terminologies of software testing. In Section 2.2, we present the basic process to build a Software Defect Prediction (SDP) model and the related research conducted in the last 50 years. In Section 2.3, we present the five most frequently used machine learning in the SDP context. In Section 2.4, we discuss the concepts and related work in the Cross-Project Defect Prediction (CPDP) context. In Section 2.5, we discuss the main factors which commonly lead to conclusion instability on related software engineering experiments. In Section 2.6, we briefly describe the concepts involved in the meta-learning literature for algorithm recommendation. In Section 2.7, we discuss the concepts of multi-label learning.

\subsection{Software Testing}

The size and complexity of software systems have grown gradually over time. In parallel, the demand for efficient procedures able to reduce cost and increase the quality of the final software product also increases.

The quality assurance term encompasses all the processes and activities conducted to aggregate quality on software (SOMMERVILLE, 2015). Although quality is always desired, the specific meaning of quality is relative to the software task and application domain. Boehm, Brown and Kaspar (1978) suggest 15 quality attributes including functional and non-functional system attributes, as presented in Table 1. Currently, the nomenclature and classification of quality attributes are standardized by ISO/IEC (2010).

For critical systems, the software reliability and related attributes are priority. In this context, three sets of activities stand out: Verification, Validation, and Test (VV\&T) 
Table 1 - Software quality attributes suggested in (BOEHM; BROWN; KASPAR, 1978).

\begin{tabular}{ccc}
\hline Safety & Understandability & Portability \\
\hline Security & Testability & Usability \\
\hline Reliability & Adaptability & Reusability \\
\hline Resilience & Modularity & Efficiency \\
\hline Robustness & Complexity & Learnability \\
\hline
\end{tabular}

(ANDRIOLE, 1986; PRESSMAN, 2009). The verification activities are designed to ensure that the development process is being conducted correctly. The validation activities provide resources to evaluate whether the software conforms with the specified requirements. Finally, the testing activities are designed to verify the correct operation of the system through its execution.

Specifically, the testing activities perform an important role in the development process since they represent the final revision of the software specification, project and codification (PRESSMAN, 2009). Even though it is not possible to ensure the complete absence of defects, the application of testing allows the early identification of defects. The earlier a defect is identified in the development process, the lower is the cost for its maintenance (BOEHM; BASILI, 2001).

Below, we present the main terminologies on software testing (IEEE, 1990):

- System Under Test (SUT): it represents the system, subsystem, or software component to be tested;

- Input domain: it represents the set of all possible values allowed as input during the running of the SUT;

- Output domain: it represents the set of all possible results produced from the running of the SUT;

- Test data: it represents an element of the input domain;

- Test case: it represents a pair input/output expected from the SUT, i.e., the test data, as input, and the expected result from its execution, as output;

- Test case suite (or test suite): it represents the set of all test cases used during a test activity;

- Oracle: it represents the decision mechanism used to recognize whether the produced output conforms with the expected output. This mechanism can be a table of values, an algorithm or even the knowledge of the tester;

- Mistake: A human action that produces an incorrect result; 
- Fault: it represents an incorrect step, process, or data definition (incorrect instruction or command);

- Error: it represents the difference between a computed, observed, or measured value or condition and the true, specified, or theoretically correct value or condition. The error is the consequence of the fault execution;

- Failure: it represents the propagation of an error to the software output. The failure is the incorrect output in relation to the software specification.

In the literature of software defect prediction (discussed in the next section) the terms fault, defect, and bug are used interchangeably (MALHOTRA, 2015).

The failures of a software are identified by running the test cases on the SUT and analyzing the produced results in relation to the oracle. In this context, the testing activities are designed aiming only to reveal the failures and faults of a software, while the debugging activities are designed to locate the revealed faults.

According to Pressman (2009), a testing activity should follow a strategy that enables management supervision as the project progresses. In addition, it should provide a script describing the steps to be conducted in a controlled manner. In order to provide a controlled and rigorous execution, the test activities are divided in four steps (BEIZER, 1990; PRESSMAN, 2009):

- Planning: in this step, an initial testing plan is designed for the conduction and management of the testing process. According to Myers, Sandler and Badgett (2011), this plan must describe all the necessary resources for the conduction of the testing process, such as: schedule, objectives, finalization criteria, human resources, tools, among others;

- Test cases design: in this step, the test cases are designed. Different techniques can be used in this step, according to the testing criteria. This step is important for the quality of the testing process since the fault coverage for the SUT is determined by the test cases (MYERS; SANDLER; BADGETT, 2011).

- Test execution: in this step, the procedures planned in the first step are executed; and

- Collection and evaluation of results: in this step, the produced results are collected, recorded, organized, and presented as reports. The results are evaluated considering the established oracle.

The steps described above refer to the strategic process of a testing activity. However, testing activities can be applied according to the life cycle of the software. According to 
Mathur (2008), during the development of a software, there is a testing level suitable for the needs of each produced artifact. For a complex software, for example, it is desirable that the test can be divided into levels able to test both the complete system and its small parts. In the literature, the testing activities can be classified in five complementary levels. These levels aim to test every artifact from the small software units to changes resulted from software maintenance. These levels are described below (PRESSMAN, 2009):

- Unit testing: in this level, all the software units are tested individually aiming at identifying the errors in the specification and/or implementation. In this context, a software unit is a component that can not be subdivided (IEEE, 1990). This testing level is focused on identifying failures in the parameters of input/output, in the data structures that influence the integrity of stored data, and in the conditions that determine the operating limits of the unit;

- Integration testing: in this level, all the software units already tested are integrated, composing subsystems. These subsystems can lead to other types of failures, such as: incorrect interface, lack or conflict of functionalities, integrity violation of files and global data structures, incorrect sequence of units, incorrect exception handling, configuration problem, and lack of resources to meet the demands of the units;

- System testing: in this level, the software is tested as a whole, considering the functional and non-functional specified requirements. In addition, this type of test evaluates the operation of the software in relation to the environment for which it was designed, including the execution platform and other external systems that are directly or indirectly related to the SUT;

- Acceptance testing: in this level, the acceptance of the generated product is verified by the end users. The test is usually performed by a group of end users who verify whether the software conforms to the functionalities previously established for the project; and

- Regression testing: in this level, the previously applied tests are reapplied to ensure the correct operation of the software for each new version. This type of test is important since changes made on specific parts of the software may compromise the entire correct operation of the software.

For each testing level presented above, it is necessary to construct a set of test cases (or test suite) that guarantees an adequate level of quality for the testing activity. In an ideal scenario, the test suite should include all elements of the input domain. However, usually, this scenario is impractical (RAPPS; WEYUKER, 1985). Therefore, the test suite must contain a finite set of test cases. In order to select the appropriate test cases, testing criteria are defined. The testing criteria can be defined using different techniques which 
establish the requirements and properties to be met by the test suite. These techniques can be grouped into three different categories, according to the information model used in the evaluation and construction of the test suite (SIMAO, 2004):

- Functional testing: in this technique, also known as black-box testing, the test cases are constructed based only on the input and output information. The implementation aspects and the internal behavior of the software are not considered. The correctness of the SUT is determined only by its specification, which defines its requirements and expected functionalities. The main classes of errors identified in this type of test are: incorrect or omitted functionality, interface errors, data structure errors, external data access errors, behavior or performance errors, initiation errors, and termination errors (PRESSMAN, 2009).

- Structural testing: in this technique, also known as white-box testing, the test cases are constructed based on the logic and functional structures of the software implementation (MYERS; SANDLER; BADGETT, 2011). In this technique, the main objective is to test procedural details of elementary parts of the software. For this, the source code information is necessary. The main classes of errors identified in this type of test are: logic faults, incorrect assumptions, design faults, and typographical faults (PRESSMAN, 2009). This technique is commonly used on the unit and integration testing levels.

- Fault-based testing: in this technique, the test cases are constructed based on the information of classes of faults known to be typical in the context of the SUT. Thus, the used information consider the characteristics of the SUT, such as the programming language, the method, or the technique used in its development. Among the main testing criteria in this category are the Mutation Analysis (WONG; MATHUR, 1995) and the Error Seeding (RAMAMOORTHY; BASTANI, 1982).

These three categories cover the main techniques known in the literature (SIMAO, 2004). However, some important techniques do not fit properly into these categories (e.g., random testing and exhaustive testing) or merge concepts from different categories (e.g., gray-box testing). The presented techniques are complementary to each other since they address different aspects of the SUT and probably reveal different classes of faults (PRESSMAN, 2009).

Another important characteristic of testing activities refers to its high cost in the development process (JONES; BONSIGNOUR, 2011). It represents the largest portion of the total cost and elapsed time spent in the development of a typical software project (MYERS; SANDLER; BADGETT, 2011). However, the consequences and the cost of not testing are even more expensive. Therefore, in a scenario with limited resources (e.g., 
financial, time, manpower), a proper management of the available resources is necessary. This issue can be mitigated with software defect prediction models, discussed in the next section.

\subsection{Software Defect Prediction}

Software Defect Prediction (SDP) models are designed to assist test practitioners on prioritizing the most defect-prone parts of the software. As a result, the available test resources can be better managed, which can reduce costs and increase the test efficiency. To achieve this objective, three different models can be constructed: classification models, to predict whether an instance is defective or not (MALHOTRA, 2015); regression models, to predict the number of defects for an instance (WEYUKER; OSTRAND; BELL, 2010); or ranking models, to predict the ordering of instances according to their defect proneness (YANG; TANG; YAO, 2015). In practice, the regression and ranking models provide similar functionalities since the outputs of regression models can be ranked according to the predicted number of defects (GAO; KHOSHGOFTAAR, 2007). In this thesis we focus on classification models although regression and ranking models are also investigated in Chapter 3. Figure 1 illustrates the general process to construct an SDP model.

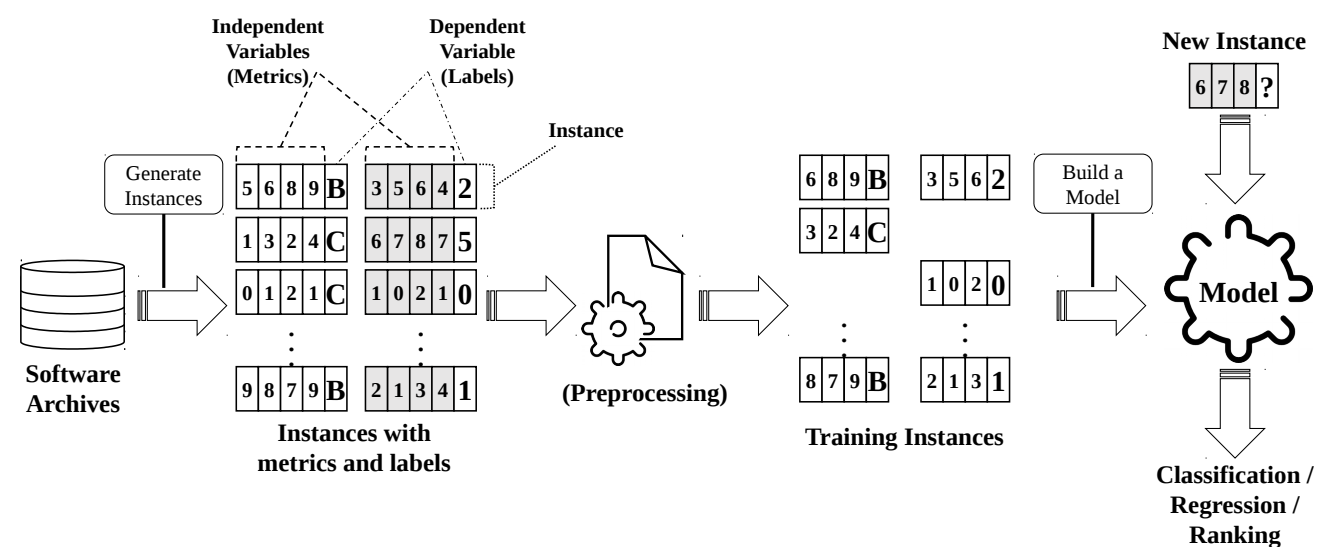

Figure 1 - General process to construct an SDP model (Adapted from (NAM, 2015)).

First, a set of historical defect data are collected to compose the training set. This training set represents the previous experiences from where the defect patterns can be learned. It is composed by a collection of instances (or examples) described by two sets of information: the independent variables and the dependent variable. Each instance can represent different levels of granularity, such as: a software component (or package), a source code file, a class, a function (or method), etc. The independent variables (also called features or attributes) characterize the properties of each instance. The dependent variable (also called target or class attribute) is the information to be learned and predicted. For classification tasks, the target attribute can assume two discrete values: defective or non-defective. For regression tasks, the target attribute represents the number of known 
defects for each instance. For ranking tasks, the target attribute represents the relative ordering of instances, not necessarily the number of defects. In the preprocessing step, some resources can be applied over the training set aiming at reducing the irrelevant, redundant and noisy information on data. Finally, a suitable machine learning algorithm is applied, generating an SDP model. Given a set of new instances, the generated SDP model should be able to predict the appropriate target values.

Despite the relative simplicity inherent to this process, several internal factors can influence the performance and applicability of an SDP model. These factors have been widely investigated in the literature and still constitute an active research area in software engineering. Figure 2 summarizes the history of the SDP literature along the last 50 years.

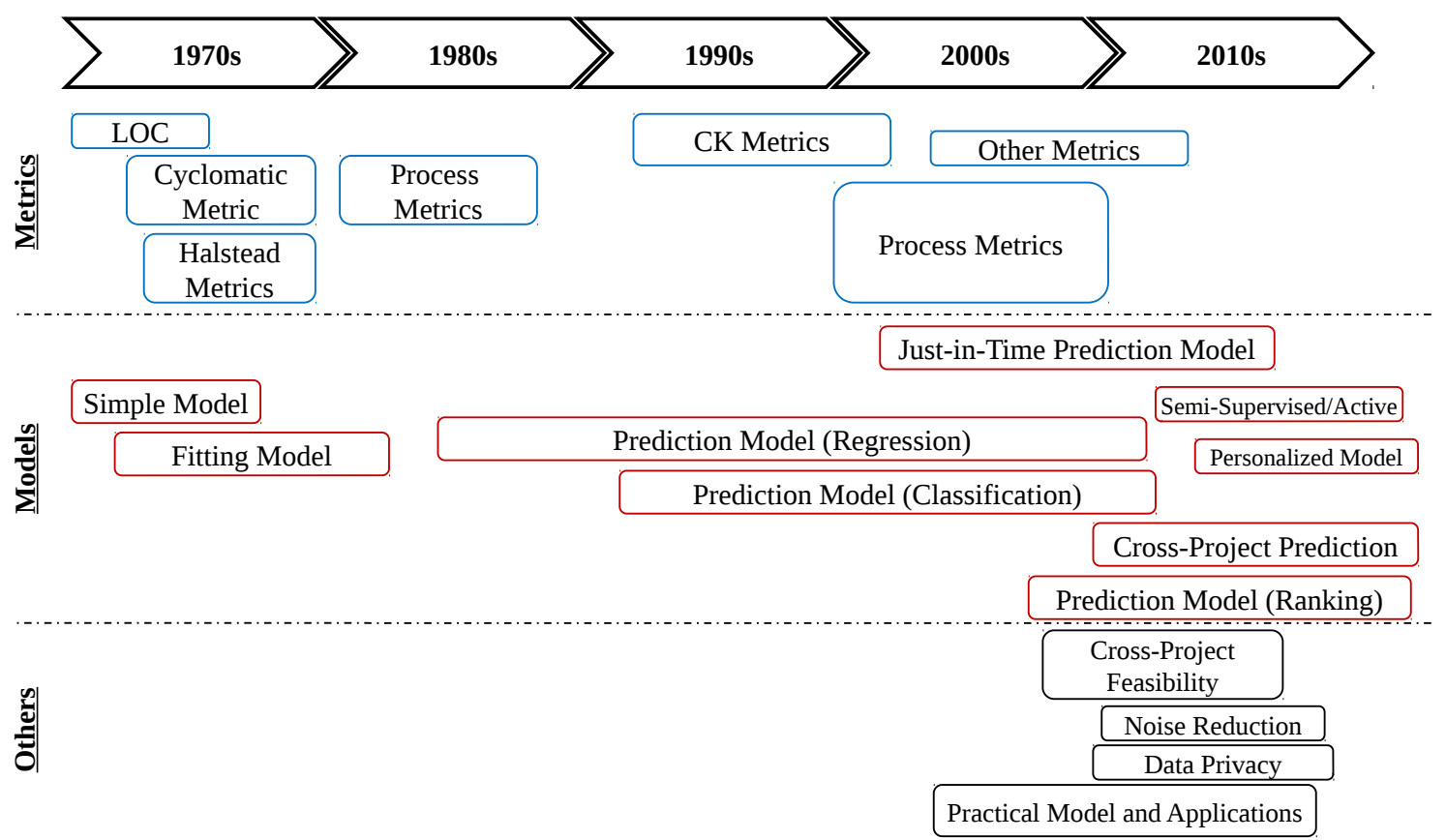

Figure 2 - Summary of the SDP literature along the last 50 years (Adapted from (NAM, 2015)).

The seminal studies in SDP focused on the correlation between complexity metrics and the number of defects (FENTON; NEIL, 1999). The first study in this context was conducted by Akiyama (1971). His work is based on the assumption that LOC (lines of code) is directly correlated to the complexity of a software and, as a consequence, to the number of defects. Later, McCabe and Halstead proposed the cyclomatic metric and the Halsted complexity metrics in McCabe (1976) and Halstead (1977), respectively. These metrics motivated a series of studies conducted in the 1970s and the early of 1980s (FENTON; NEIL, 1999).

The use of regression models to predict defects started with Shen et al. (1985). They constructed a linear regression model based on process metrics and tested it on new program modules. Munson and Khoshgoftaar (1992) proposed a binary classification model, also based on process metrics, to classify the software modules into: high risk or 
low risk. The classification model achieved $92 \%$ of accuracy on the tested subject system. Besides the positive results, both studies presented limitations. For example, Shen et al. (1985) pointed out that errors fixed informally in the unit test phase were not collected. Munson and Khoshgoftaar (1992) also pointed out the few resources available for the extraction of development process data.

Further, Chidamber and Kemerer (1994) proposed a suite of complexity metrics for object-oriented systems, called CK metrics. These metrics were evaluated in the context of SDP by Basili, Briand and Melo (1996). In parallel, in the 1990s the software companies started to adopt tools to document the development process, such as the version control systems. As a result, more software data became available. This event stimulated the proposition of several process metrics from the middle of the 2000s (BACCHELLI; D'AMBROS; LANZA, 2010; BIRD et al., 2011; D‘AMBROS; LANZA; ROBBES, 2012; HASSAN, 2009; LEE et al., 2011; MOSER; PEDRYCZ; SUCCI, 2008; NAGAPPAN; BALL, 2005; RAHMAN et al., 2011).

Ostrand, Weyuker and Bell (2004) proposed the use of code metrics and historical defect information for defect prediction in large-scale industrial systems. Zimmermann and Nagappan (2008) proposed the use of social network analysis metrics, extracted from the software dependency graph. Further, other metrics were investigated, such as developer-related metrics (PINZGER; NAGAPPAN; MURPHY, 2008), organizational metrics (NAGAPPAN; MURPHY; BASILI, 2008), change related metrics (HERZIG et al., 2013), and antipatterns related metrics (TABA et al., 2013).

Weyuker, Ostrand and Bell (2010) compared four regression algorithms in the ranking context over three large industrial software systems. Usually, the ranking task is conducted in two steps (GAO; KHOSHGOFTAAR, 2007; DENARO; PEZZè, 2002; WEYUKER; OSTRAND; BELL, 2010): first, a regression model is build and applied in order to predict the number of defects for each instance; then, the instances are ordered based on their predicted number of defects. Recently, Yang, Tang and Yao (2015) proposed a learning-to-rank approach in which the model is constructed aiming at directly optimizing the ranking performance, instead of the two step approach.

The better understanding of SDP also revealed its limitations. For example, in the traditional SDP, a software can not be predicted before release since the independent variables are extracted from the final product. This earlier feedback could increase the positive impacts on the software quality. The Just-In-Time (JIT) defect prediction models are constructed from the history of changes on software (MOCKUS; VOTTA, 2000). This different approach allows the prediction of defects whenever a change is made on the source code. The JIT defect prediction has been widely investigated in recent years (FUKUSHIMA et al., 2014; KAMEI et al., 2013; KIM; WHITEHEAD JR.; ZHANG, 2008; TAN et al., 2015). Peters and Menzies (2012) investigated the privacy issue and concluded 
that privatizing the data do not influence the efficacy of SDP models. Jiang, Tan and Kim (2013) proposed a personalized defect prediction model considering the different patterns inherent to the different developers.

Most of the studies mentioned so far share the same methodology, called WithinProject Defect Prediction (WPDP). In this methodology, the training set is composed by instances from the same software. Therefore, the prediction of a software is based on its previous versions. However, an appropriate amount of historical defect data may not be available. An alternative is to compose the training set with external known projects. This methodology is called Cross-Project Defect Prediction (CPDP). This research topic is addressed in details in Section 2.4.

In parallel, the emergence of new machine learning techniques led to new studies in the SDP context. Lu and Cukic (2012) and Li et al. (2012) investigated the use of active learning and semi-supervised learning to address the lack of historical defect data in the traditional WPDP. In their approaches, a small sample of instances is initially labeled with the support of an oracle (human professionals). This small sample is then iteratively increased in a process combining semi-supervised learning, supervised learning and active learning. Even though the human effort becomes necessary, their approaches eliminate the need for historical data.

Other studies focused on case studies and the impact of SDP models on the industry. Engström, Runeson and Wikstrand (2010) investigated the efficiency of SDP models applied for prioritizing regression tests in a major company of embedded systems. Bell, Weyuker and Ostrand (2011) discussed how to assess the impact of the incorporation of defect predictions into the development process. Their analysis considers a series of previous experiments involving SDP models applied to large-scale industrial systems.

In the same context, part of the literature focused on the performance of machine learning algorithms applied to SDP. Lessmann et al. (2008) compared the performances of 22 classifiers over 10 public domain datasets. Their results show no statistically significant difference among the top 17 classifiers. Later, Ghotra, McIntosh and Hassan (2015) revisited these experiments considering two different collections of data. Opposite to the previous results from Lessmann et al. (2008), Ghotra, McIntosh and Hassan (2015) concluded that the used classification technique had a significant impact on the performance of defect prediction models. The implications of these contrasting results are discussed in Section 2.5. In this thesis, we address five distinct machine learning algorithms, presented in the next section. 


\subsection{Machine Learning Algorithms for Software Defect Prediction}

Each machine learning algorithm has an inductive bias (MITCHELL, 1997). This bias is a result of all the assumptions that an algorithm makes in order to generalize from the training data to unseen instances. Therefore, the inductive bias inherent for each algorithm makes it more (or less) suitable for a specific application domain.

Malhotra (2015) presents a systematic literature review on machine learning techniques for software defect prediction, based on 64 primary studies (from 1991 to 2013). In their review were identified the five most frequently used classifiers: Support Vector Machines; Multilayer Perceptron; Naïve Bayes; C4.5; and Random Forest. All these five classifiers are addressed in this work. We briefly describe each classifier below. Detailed information can be found in a specialized machine learning literature (MITCHELL, 1997; MAIMON; ROKACH, 2010; BRAZDIL et al., 2017).

\subsubsection{Support Vector Machines}

A Support Vector Machine (SVM) model aims at finding the optimal hyperplane that maximally separates samples in two different classes. This classifier is also robust to redundant and irrelevant features since the number of features does not affect the complexity of an SVM model. Given a training set $E$ with $n$ tuples $\left(\mathbf{x}_{i}, y_{i}\right)$, where $\mathbf{x}_{i} \in \mathbb{R}^{d}$, $y_{i} \in\{-1,+1\}$, and $d$ is the dimensionality of each instance, the training phase of an SVM aims at finding the linear classifier $g(\mathbf{x})=\operatorname{sgn}(\mathbf{w} \cdot \mathbf{x}+b)$ able to separate the instances of $E$ with minimum error and separate the classes of $E$ with maximum margin $\rho$.

Given a linear function $f(\mathbf{x})=\mathbf{w} \cdot \mathbf{x}+b$, the margin $\rho\left(\mathbf{x}_{i}, y_{i}\right)$ used to classify the pattern $\mathbf{x}_{i}$ is given by $y_{i} f\left(\mathbf{x}_{i}\right)$. It calculates the distance of instance $\mathbf{x}_{i}$ in relation to the hyperplane separating the classes. The margin $\rho$ of the linear classifier $f$ is defined as the minimum margin observed within the training set. To maximize $\rho$ is equivalent to minimize $\mathbf{w}$ (HEARST, 1998). Thus, the search can be to find a hyperplane with $\|\mathbf{w}\|$ small while keeping $\rho$ fixed and ensuring that no training instance has margin lower than $\rho$ (SCHOLKOPF; SMOLA, 2001). This search leads to the following optimization problem:

$$
\min \left\{\|\mathbf{w}\|^{2}+C \sum_{i=1}^{n} \xi_{i}\right\} \text { such that } y_{i}\left(\mathbf{w} \cdot \mathbf{x}_{i}-b\right) \geq 1-\xi_{i} \text { and } 1 \leq i \leq n
$$

where $C$ represents the trade-off between the generalization capability of the SVM and the width of the margin $\rho$, and $\xi$ is the variable responsible for the relaxation of constraints, i.e., it allows some patterns having margin lower than $\rho$. 
This problem can be solved by Quadratic Programming (HEARST, 1998), or other strategies (HAYKIN, 1998). After solved, this optimization problem leads to a classifier given by Equation 2.2 .

$$
g(\mathbf{x})=\operatorname{sgn}\left(\sum_{\mathbf{x}_{i} \in S V s} \alpha_{i} y_{i} \mathbf{x}_{i} \cdot \mathbf{x}+b\right),
$$

where $\alpha_{i}$ are the variables resulting from the optimization process, the $S V s$ are the Support Vectors representing the training instances more close to the separating hyperplane. The $S V s$ are considered the more informative data from the training set since the final classifier is determined by the limits associated to the $S V s$. Figure 3 illustrates an example of linearly separable data trained with an SVM.

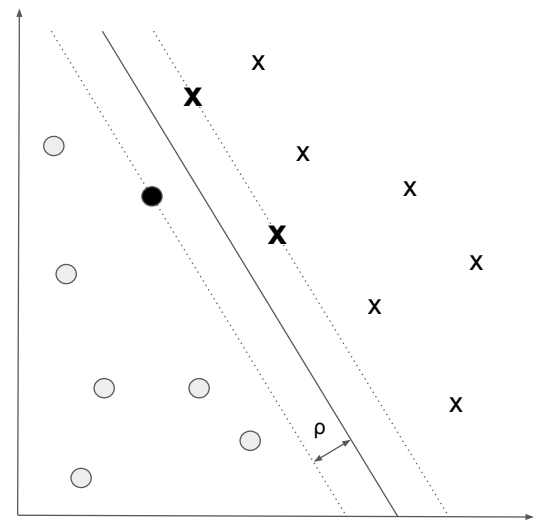

Figure 3 - Example of an SVM applied to a set of linearly separable data. The highlighted instances represent the Support Vectors, the center line represents the hyperplane and the dashed lines represent the margins.

In this work, we consider an SVM variation called Sequential Minimal Optimization (SMO). This technique optimizes the SVM training by dividing the large Quadratic Programming problem in a series of possible Quadratic Programming problems (PLATT, 1999).

\subsubsection{Multilayer Perceptron}

Multilayer Perceptron (MLP) is a neural network model based on the backpropagation algorithm (HAYKIN, 1998). The MLP consists of three or more layers (an input and an output layer with one or more hidden layers) of nodes in a directed graph (see Figure 4). Each layer is fully connected to the next one. The nodes (except for the input nodes) are neurons (or processing elements) with a nonlinear activation function. The weights of each node in the network are iteratively updated in an attempt to minimize a loss function calculated from the output layers. This procedure defines the back-propagation algorithm, which can be considered a generalization of the least-mean-square algorithm on a linear perceptron network (HAYKIN, 1998). 


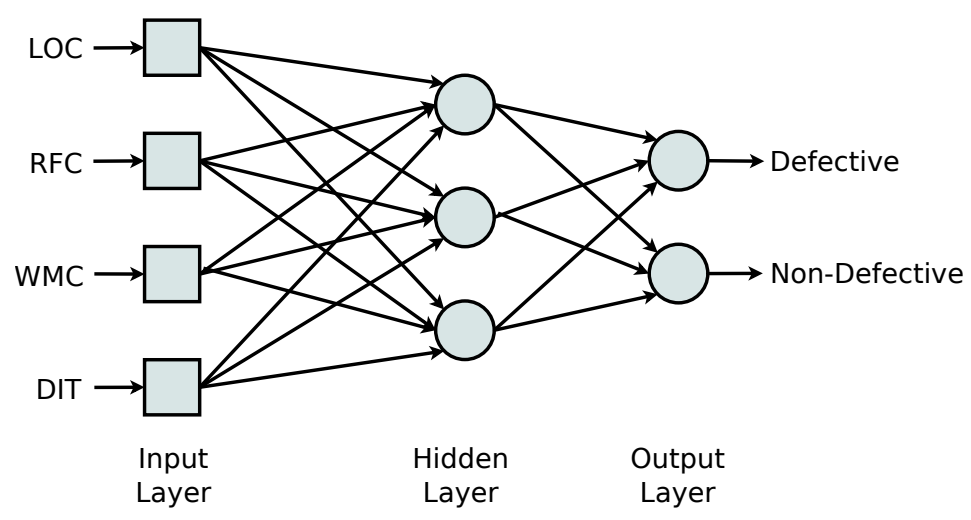

Figure 4 - Example of an MLP network.

The MLP models are robust to noise and high dimensional data. In contrast, the proper parameter configuration and the interpretation of the produced model are difficult issues for MLP models.

\subsubsection{Naïve Bayes}

Naïve Bayes (NB) is a simple statistical algorithm based on Bayes' Theorem. In the defect prediction context, this theorem can be defined as follows:

$$
P\left(c_{k} \mid x\right)=\frac{P\left(c_{k}\right)}{P(x)} \prod_{j=1}^{m} P\left(x_{j} \mid c_{k}\right),
$$

where $c_{k}$ is an element of the set of class values (defective or non-defective), $x=\left(x_{1}, \ldots, x_{m}\right)$ is a features vector, $P\left(c_{k}\right)$ and $P(x)$ are respectively the prior probabilities of $c_{k}$ and $x$ occur, and $P\left(x_{j} \mid c_{k}\right)$ is the probability of $x_{j}$ given $c_{k}$. These probabilities are combined based on the training set. The theorem calculates the posterior probability of $c_{k}$ given that $x$ is true. The term "naïve" is due to its assumption that the features are independent. Although this assumption is not always true, this algorithm has been reported as an efficient classifier for defect prediction (LESSMANN et al., 2008).

\subsubsection{C4.5}

C4.5 (C45) is a decision tree algorithm which extends the Quinlan's earlier ID3 algorithm. A decision tree is a collection of decision rules defined in each node. The tree is generated by associating to each node the feature that most effectively split the set of training data. The more able to separate defective from non-defective instances, the more effective is a split. The splitting criterion is the normalized information gain (MAIMON; ROKACH, 2010). This criterion quantifies how clean is the separation of labels after adding the extra split in relation to before adding it. The construction of the tree model start off with a single node containing all observations. Then, after each split, the data 
points move down to their respective branch. The classification of instances is performed by following a path trough the tree from the root to the leaf nodes where a class value is taken. Figure 5 illustrates a C4.5 tree model constructed from 12 hypothetical instances of software.

\begin{tabular}{ccc}
\hline LOC & RFC & Class \\
\hline 16 & 5 & ND \\
\hline 16 & 25 & D \\
\hline 16 & 15 & D \\
\hline 25 & 4 & ND \\
\hline 26 & 7 & ND \\
\hline 26 & 32 & ND \\
\hline 26 & 16 & D \\
\hline 33 & 6 & D \\
\hline 50 & 7 & ND \\
\hline 50 & 16 & ND \\
\hline 50 & 35 & ND \\
\hline 50 & 35 & ND \\
\hline
\end{tabular}

(a)

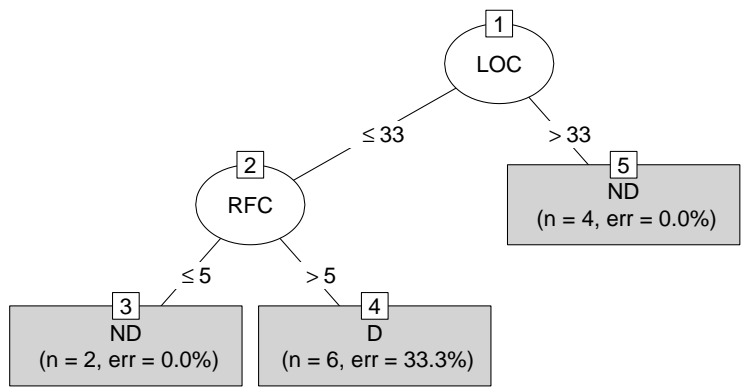

(b)

Figure 5 - Example of a C4.5 tree model generated for 12 hypothetical instances. The target attribute contains two possible labels (Defective or Non-Defective).

This algorithm is robust for irrelevant attributes since they are ignored in the model construction process. This algorithm also produces an interpretable model and demands low computational cost in relation to other classifiers.

\subsubsection{Random Forest}

Random Forest (RF) is an ensemble technique based on a collection of decision trees (BREIMAN, 2001). This algorithm is based on a technique called bagging in which each tree is constructed from a different subset of the original training set. First, a subset of features is randomly selected from the original training set and, then, a subset of instances is randomly selected (with replacement) to compose the final dataset used for each tree. This technique creates a variety of tree models with different inductive biases. The final prediction for a new instance is given by the majority voting of all trees (for classification) or the mean prediction (for regression) (BREIMAN, 2001). Figure 6 illustrates the simplified architecture of a Random Forest model. This algorithm is robust to redundant and irrelevant features although it can produce overfitting models.

\subsection{Cross-project Defect Prediction}

The dependent variable also represents an important role in software defect prediction. Actually, the learning is only possible when the defect data is available. However, in practice, not all software companies maintain clear records about historical defect data or sufficient data from previous projects (KITCHENHAM; MENDES; TRAVASSOS, 2007). In this case, the training set can be composed by external projects with 


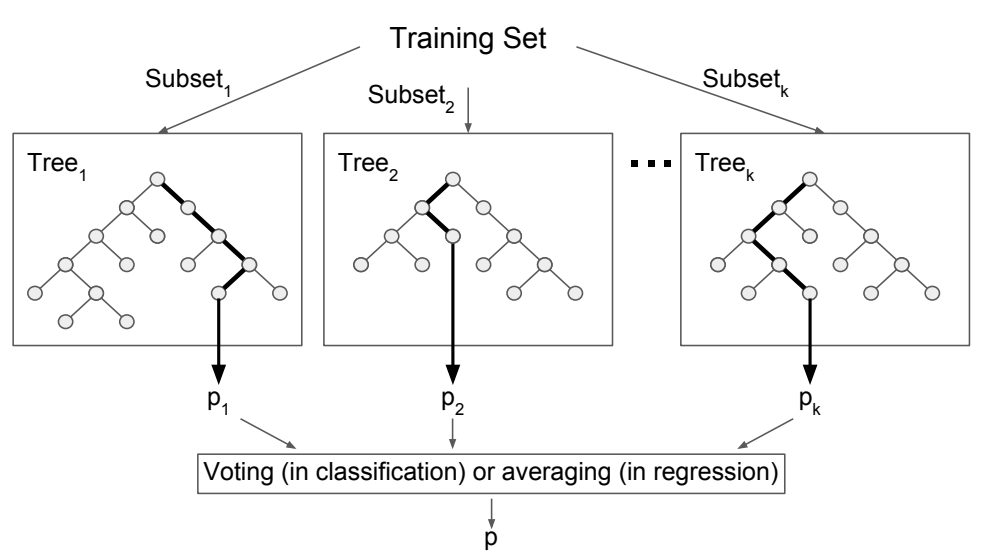

Figure 6 - Example of a Random Forest model.

known defect information. This approach is called Cross-Project Defect Prediction (CPDP) (GUNARATHNA; TURHAN; HOSSEINI, 2016). On the one hand, it solves the lack of historical defect data. On the other hand, it introduces heterogeneity on data, which may decrease the efficiency of defect prediction models (ZIMMERMANN et al., 2009).

In a recent systematic literature review, Gunarathna, Turhan and Hosseini (2016) highlighted the contributions of 46 primary studies on CPDP from 2002 to 2015. Part of these works focused on the feasibility of CPDP. Briand, Melo and Wust (2002) conducted one of the earliest studies on this topic and verified that CPDP models outperform random models in the studied case. Zimmermann et al. (2009) conducted a large scale experiment in which they evaluated 622 pairs of cross-project models ( project $_{1}$, project $_{2}$ ). They found that a model trained with project $_{1}$ was able to predict project $_{2}$ with good performance in only $3.4 \%$ of pairs.

Other studies focused on possible solutions to mitigate the heterogeneity of the data and improve the learning capacity of CPDP models. Among all solutions, we highlight the transfer learning solutions ${ }^{1}$ (PAN; YANG, 2010). In this approach, the solutions use some knowledge about the target domain in order to approximate the different distributions of source and target data.

Figure 7 illustrates the difference between traditional machine learning and transfer learning. The traditional machine learning assumes the same distribution for training and test data. Thus, a distinct model is constructed for each distribution or application domain. However, collecting and labeling new data is costly (PAN; YANG, 2010). The transfer

1 The context of this work fits in a special case of transfer learning, called domain adaptation (PAN; YANG, 2010). In this case, source (external projects) and target (project to be predicted) domains may be different while sharing the same learning task. In addition, the target domain may be unlabeled (e.g., the first release of a software project, with no historical defect data). 


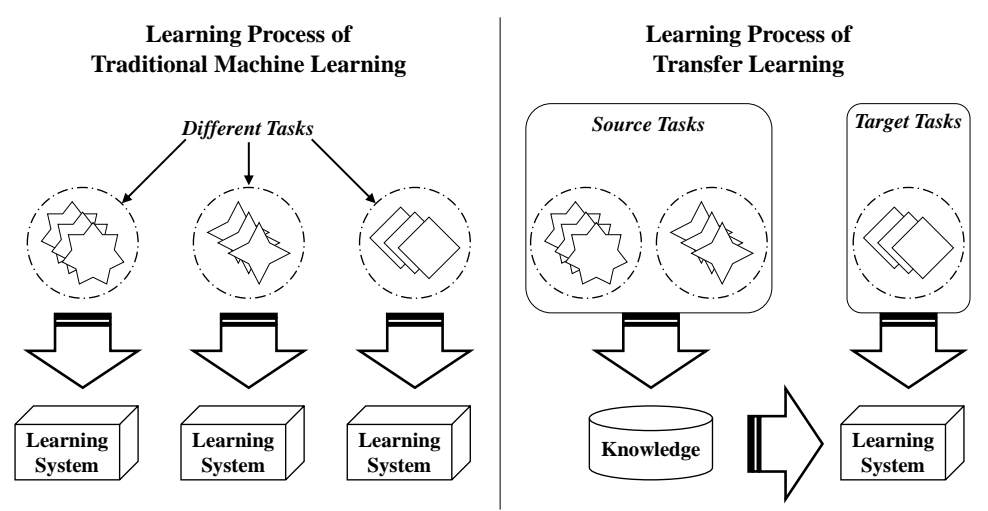

Figure 7 - Different learning process between traditional machine learning and transfer learning (Adapted from (PAN; YANG, 2010)).

learning methods allow transferring knowledge from a domain with enough training data to another domain with few training data.

At least three different strategies can be identified among the transfer learning solutions: transformation of data (WATANABE; KAIYA; KAIJIRI, 2008; CRUZ; OCHIMIZU, 2009; NAM; PAN; KIM, 2013; ZHANG et al., 2014); filtering a subset of the training data (TURHAN et al., 2009; JURECZKO; MADEYSKI, 2010; HERBOLD, 2013; HE et al., 2013); and weighting the training data according to the target data (MA et al., 2012).

Watanabe, Kaiya and Kaijiri (2008) proposed to standardize the target data based on the source mean values. Cruz and Ochimizu (2009) proposed a similar approach but based on the median values, associated with power transformation. Nam, Pan and Kim (2013) proposed to project both source and target data in a common feature space by means of Transfer Component Analysis (TCA). Moreover, they investigated the impact of min/max and Z-score standardizations applied over source and target data. Zhang et al. (2014) proposed a universal defect prediction model based on clusters created using the project context.

Turhan et al. (2009) proposed to use the K-Nearest Neighbor (KNN) filter to select the most similar training instances in relation to the target instances. Jureczko and Madeyski (2010) proposed to perform clustering in order to identify the most suitable training projects for a target project. Peters, Menzies and Marcus (2013) proposed to use clustering to filter the closest training instances in relation to the target data. Herbold (2013) proposed to use the k-nearest neighbor filter but to select the most similar projects (instead of individual instances). He et al. (2013) proposed to select the most similar projects but considering the separability of each project in comparison to the target data. Moreover, they proposed to use the separability strategy to filter unstable features. Lastly, they applied an ensemble approach over the selected projects with filtered features.

Ma et al. (2012) used the idea of data gravitation to prioritize and weight the training data for the Naïve Bayes classifier based on its similarity to the target data. 
To the best of our knowledge, no extensive comparison of transfer learning methods were conducted in the CPDP literature. Commonly, authors compared their new solutions either with the KNN filter (e.g., Ma et al. (2012), He et al. (2013), Herbold, Trautsch and Grabowski (2016)) or some baseline (e.g., Herbold (2013), Nam, Pan and Kim (2013), Herbold, Trautsch and Grabowski (2016)). The baseline, usually, is defined as the direct application of a machine learning technique, with no CPDP treatment.

In the systematic literature review presented in Gunarathna, Turhan and Hosseini (2016), the authors compared the performance of CPDP methods based on the information provided by each published work. However, the considered studies diverge in terms of data, classifiers, and performance measures. The absence of a uniform experimental setup precludes concrete comparisons of performances between studies on a large scale. This issue is better discussed in the next section.

\subsection{Conclusion Instability}

Defect prediction models assist testers on prioritizing test resources. Yet, there is another important decision in this process concerning which model is the most suitable for a specific domain. This decision is difficult for two main reasons. First, as stated in Shepperd, Bowes and Hall (2014), no single defect prediction technique dominates. This statement has support in the theorem known as no free lunch, which says that there is no inductive bias suitable for all domains (WOLPERT, 2002). Second, because of the conclusion instability found in the experimental software engineering (MENZIES; SHEPPERD, 2012). Not rarely, different studies on the same subject produce conflicting conclusions. For example, Kitchenham, Mendes and Travassos (2007) reviewed empirical studies applied to effort estimation in which local data are evaluated in relation to data imported from other organizations. From the seven reviewed studies, three concluded that imported data are not worse than local data, while four studies concluded that imported data are significantly worse than local data.

Part of this conclusion instability is credited to the bias produced by the different research groups (SHEPPERD; BOWES; HALL, 2014). However, in a recent study, Tantithamthavorn et al. (2016) argued that this bias is actually due to the strong tendency of a research group to reuse experimental resources such as datasets and metrics families.

Menzies and Shepperd (2012) also credited the conclusion instability to the variance between similar experiments. This variance is related to the internal resources and procedures used in each experimentation. Among the suggestions proposed by Menzies and Shepperd (2012) to reduce the conclusion instability is the use of a uniform experimental setup. In the context of CPDP, several factors can influence the performance analysis, such as: 
- The collection of software projects used as training and test sets: different data repositories are used in the literature of CPDP, such as PROMISE, Apache, Eclipse, and NASA MDP (TURHAN et al., 2009; HERBOLD, 2013; HE et al., 2015);

- The independent variables set: some of the data repositories mentioned above provide datasets with different sets of metrics. The set of metrics is an important source of performance variability (TANTITHAMTHAVORN et al., 2016);

- The modeling technique: although some solutions are intrinsically associated to some classifier (e.g., Ma et al. (2012)), other transfer learning solutions are independent of the learning algorithm (e.g., Watanabe, Kaiya and Kaijiri (2008), Turhan et al. (2009), Herbold (2013)) and their performance can be influenced by the applied classifier;

- The cross-project configuration: one-to-one, when only one dataset (or software project) is used for training (ZIMMERMANN et al., 2009); or many-to-one, when multiple projects compose the training set (TURHAN et al., 2009);

- Data preprocessing: different methods can be applied in the preprocessing step, such as normalization, standardization, feature selection, or data transformation (MAIMON; ROKACH, 2010). These methods modify the value range, distribution and scale of data, interfering directly on the CPDP model performance (KEUNG; KOCAGUNELI; MENZIES, 2013; NAM; PAN; KIM, 2013); and

- Performance measure: some traditional metrics (e.g., precision, recall, f-measure) are sensitive to the threshold that separates a defective from a non-defective instance. Other metrics (e.g., AUC), however, are unrestricted to this threshold. Furthermore, different metrics focus on different aspects of the performance. Thus, a proper performance comparison demands the use of equivalent measures.

Considering each of these bias factors mentioned above, in Chapter 4, we define a uniform experimental setup for the performance comparison of $31 \mathrm{CPDP}$ methods ${ }^{2}$ derived from the combination of six state-of-the-art transfer learning solutions with the five most popular classifiers for defect prediction.

In order to mitigate conclusion instability, Menzies and Shepperd (2012) also suggest the investigation of approaches able to learn the properties of a particular domain and, as a result, suggest a suitable learner. This suggestion is strongly related to the meta-learning architecture proposed and evaluated in Chapter 5. We discuss the background of this proposal in the next section.

2 In this work, the therm CPDP method refers to the entire process (transfer learning solution + classifier) which leads to the prediction model. 


\subsection{Meta-learning for Algorithm Recommendation}

Considering the no free lunch theorem and the concept of conclusion instability discussed above, automated resources to assist practitioners in the difficult task of choosing a suitable model for a specific domain are desirable. This task is related to the algorithm recommendation task investigated in the meta-learning literature (LEMKE; BUDKA; GABRYS, 2015). The learning capacity of a traditional model (here identified as base-learner) is limited to its inductive bias (MITCHELL, 1997). A meta-learning model, however, is characterized by its capacity of learning from previous experiences and adapting its inductive bias dynamically according to the target domain (BRAZDIL et al., 2017). In other words, a meta-learning model is designed to learn in which conditions a given solution is more suitable to be applied instead of others.

The main challenge in the algorithm recommendation task is to discover the relationship between measurable features of the problem (or dataset) and the performance of different solutions (algorithms, hyper-parameters, etc.) (RICE, 1976). In the literature, this task is approached as a typical classification problem, although at a meta-level (KALOUSIS, 2002; VILALTA; DRISSI, 2002; BRAZDIL; SOARES; COSTA, 2003; ALI; SMITH, 2006). Figure 8 illustrates the general process of a meta-learning system for algorithm recommendation.
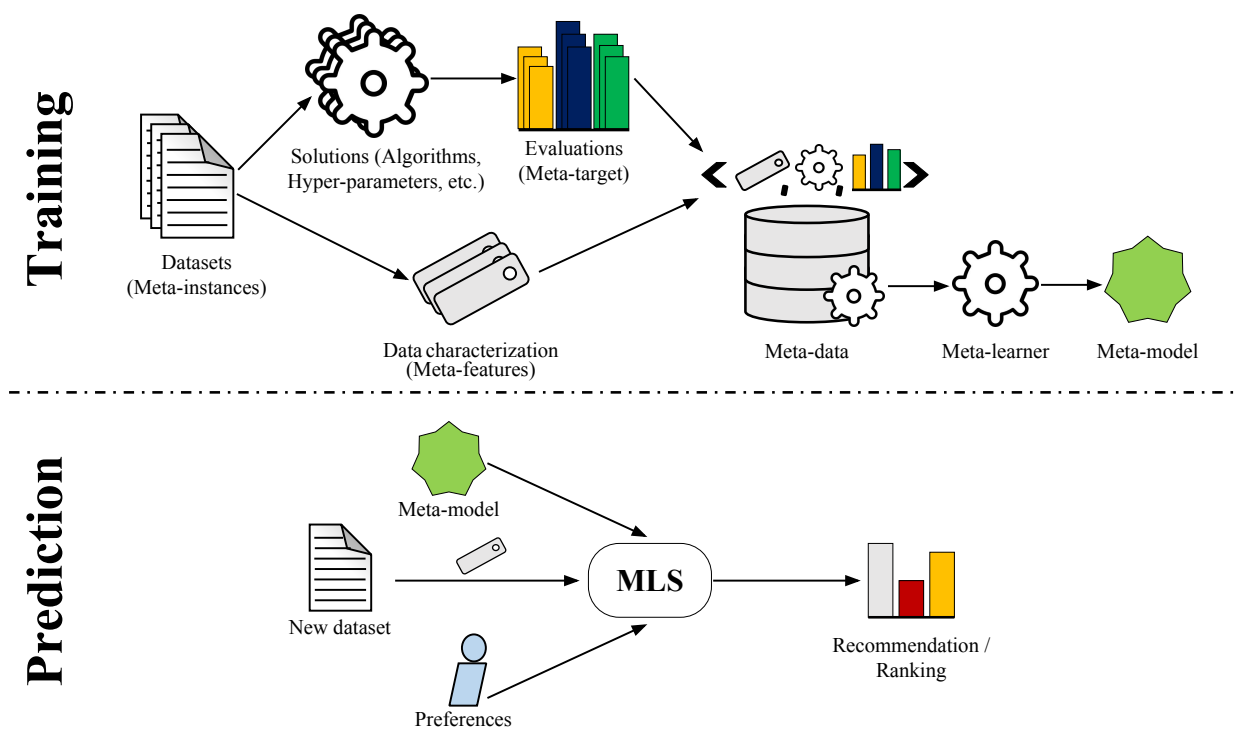

Figure 8 - General process of a meta-learning system for algorithm recommendation (Adapted from (SERBAN et al., 2013)).

Similar to the traditional learning task, the success of a meta-model depends on two main factors: 1) building an accurate meta-data; and 2) applying a suitable machine learning algorithm to compose the meta-learner. The meta-data is represented by a table of meta-instances. Each meta-instance represents a dataset (i.e., a previous learning task experience); the independent variables (or meta-features) characterize each dataset; and 
the dependent variable (or meta-target) represents the goal of the meta-learning task.

Different goals have been explored in the literature for both classification and regression tasks. In classification tasks, the meta-target is commonly associated to a label representing the solution with highest performance (or lower cost). Examples of meta-learning classification tasks are: to predict a suitable base-learner (DORES et al., 2016); to predict a suitable feature selection method (PARMEZAN; LEE; WU, 2017); to predict if the tuning of hyper-parameters for SVMs is beneficial or not (MANTOVANI et al., 2015); among others (LEMKE; BUDKA; GABRYS, 2015). In regression tasks, each meta-target represents the continuous value of performance (or cost) obtained by a specific solution. In this context, the meta-learning task is commonly designed to produce a rank of possible solutions (BRAZDIL et al., 2017). The recommended order can be useful either to guide the decision task (KANDA et al., 2016) or to compose ensemble solutions (CRUZ; SABOURIN; CAVALCANTI, 2017).

Linked to the goal of the meta-learning task is the set of meta-features. Traditionally, they are classified into five main categories (BRAZDIL; HENERY, 1994; REIF et al., 2014): general features, obtained directly from the properties of the dataset (e.g., number of instances, number of features); statistical features, obtained from statistical measures (e.g., correlation between features); information-theoretic features, typically obtained from entropy measures (e.g., class entropy, feature entropy); model-based features, extracted from internal properties of an applied model (e.g., the width and height of a decision tree model); and landmarking features, obtained from the resulting performance of simple classifiers (e.g., the accuracy obtained from a 10-fold cross-validation with 1-nearest neighbor classifier).

In the context of this study, for example, all datasets are composed by the same set of continuous features (see Section 4.2.1). In addition, the target data may be unlabeled (see Section 5.2.2). These two characteristics invalidate most of the traditional meta-features, since many of them are related to discrete features, to the dimensionality of the dataset, or are label dependent - including information-theoretic, model-based and landmarking features.

A viable alternative is to characterize the datasets based on unsupervised metafeatures (non-dependent on the target attribute). However, this alternative is little explored in the algorithm recommendation literature. Although some of the traditional meta-features are not dependent on the target attribute, they are commonly addressed associated with label dependent meta-features. In a recent study, Santos and Carvalho (2016) proposed a set composed of 53 meta-features applied in the context of active learning, including only unsupervised features such as general features, statistical features and clustering based features. Another related approach is to represent a dataset based on its distributional characteristics (e.g., mean, maximum and standard deviation of all features). Although this 
latter approach is not commonly applied for algorithm recommendation tasks, it is already applied in the context of CPDP with different purposes (HE et al., 2012; HE et al., 2013; HERBOLD, 2013). For example, Herbold (2013) uses the distributional characteristics of datasets to filter the most similar projects to compose the final training set. These two unsupervised approaches are explored and evaluated in this study. A better description is provided in Section 5.2.2.

In principle, any machine learning algorithm can be used at the meta-level. However, a common aspect of meta-learning tasks is the scarce set of training data (BRAZDIL et al., 2017). This issue is mitigated in the literature with lazy learning methods, such as k-nearest neighbor classifier (LEMKE; BUDKA; GABRYS, 2015; BRAZDIL et al., 2017), although other types of models have been successfully applied (e.g., neural networks, random forest, ensembles) (DORES et al., 2016; PARMEZAN; LEE; WU, 2017).

In Dores et al. (2016), the authors proposed a meta-learning framework for algorithm recommendation in the context of WPDP. Their meta-learning solution includes a meta-data composed of seven classifiers applied over 71 distinct datasets. The datasets are characterized with traditional meta-features, as described above. They evaluated two meta-learners: a Random Forest model; and a majority voting ensemble, including all seven classifiers. Their experiments reveal a better performance obtained with the meta-learning solution across datasets in relation to each of the seven classifiers applied individually. However, no statistical analysis was reported.

In Chapter 5, we propose and evaluate a different meta-learning solution designed specifically for the CPDP context. The particularities of this context lead to new issues, carefully investigated and presented in this thesis. Among these issues we can highlight the unsupervised meta-features set and the multi-label context (see Section 2.7). Furthermore, a series of other factors distinguish the proposed solution from previous meta-models, including the collection of datasets, the validation process, the performance measure, the feature selection procedure, and the statistical analysis.

\subsection{Multi-label Learning}

The meta-learning solution proposed in this work also configures a multi-label learning task since each meta-instance can be associated to more than one suitable CPDP method. In this section we present the main concepts related to this particular domain.

In the traditional single-label classification task, as described in Section 2.2, each instance of a dataset is associated with a single label $\lambda$ from a set of disjoint labels $\mathscr{L}=\left\{\lambda_{1}, \ldots, \lambda_{c}\right\}$, where $c=|\mathscr{L}|$ and $c>1$. The learning task is called binary classification when $c=2$ and multi-class classification when $c>2$. In a multi-label classification task, each instance is associated with a set of labels $L \subseteq \mathscr{L}$ (TSOUMAKAS; KATAKIS, 2007). 
Table 2 - Multi-label training set.

\begin{tabular}{ccccccccc}
\hline \multirow{2}{*}{ Instances } & \multicolumn{4}{c}{ Features } & \multicolumn{4}{c}{ Labels (Classes) } \\
\cline { 2 - 9 } & $F_{1}$ & $F_{2}$ & $\ldots$ & $F_{m}$ & $\lambda_{1}$ & $\lambda_{2}$ & $\ldots$ & $\lambda_{c}$ \\
\hline$I_{1}$ & $x_{11}$ & $x_{12}$ & $\ldots$ & $x_{1 m}$ & $y_{11}$ & $y_{12}$ & $\ldots$ & $y_{1 c}$ \\
$I_{2}$ & $x_{21}$ & $x_{22}$ & $\ldots$ & $x_{2 m}$ & $y_{21}$ & $y_{22}$ & $\ldots$ & $y_{2 c}$ \\
$\vdots$ & $\vdots$ & $\vdots$ & $\ddots$ & $\vdots$ & $\vdots$ & $\vdots$ & $\ddots$ & $\vdots$ \\
$I_{n}$ & $x_{n 1}$ & $x_{n 2}$ & $\ldots$ & $x_{n m}$ & $y_{n 1}$ & $y_{n 2}$ & $\ldots$ & $y_{n c}$ \\
\hline
\end{tabular}

Let $\mathbf{x}_{i} \in \mathbb{R}^{m \times 1}$ be a real value instance vector, $\mathbf{y}_{i} \in\{0,1\}^{c \times 1}$ a label vector for $\mathbf{x}_{i}, n$ the number of training instances and $\mathbf{y}_{j}$ the $j$ th label of the $i$ th instance, where $1 \leq i \leq n$ and $1 \leq j \leq c$. The element $\mathbf{y}_{i j}$ is 1 (or 0 ) when the $j$ th label is relevant (or irrelevant) for the $i$ th instance. We denote $L_{i}$ as the set of relevant labels for the instance $\mathbf{x}_{i}$. A training set is composed by the instance matrix $X \in \mathbb{R}^{n \times m}$ and the label matrix $Y \in\{0,1\}^{n \times c}$ (see Table 2).

Given a multi-label training set $(X, Y)$, the learning task can have two goals: classification or ranking (SCHAPIRE; SINGER, 2000). A multi-label classification model is defined by $H: \mathbb{R}^{m} \rightarrow\{0,1\}^{c}$. A multi-label ranking model is defined by $F: \mathbb{R}^{m} \rightarrow \mathbb{R}^{c}$, where the predicted value can be regarded as the confidence of relevance. The ranking is given by ordering the resulting confidence of relevance. We can also define a multi-label classifier $F$ that assigns a single label for $\mathbf{x}_{i}$ by setting arg $\max F\left(\mathbf{x}_{i}\right)$. In other words, the predicted single label for $\mathbf{x}_{i}$ is given by the top ranked confidence of relevance generated by $F$.

Several methods have been proposed in the literature of multi-label learning (MAIMON; ROKACH, 2010). The existing methods can be grouped into two main categories: 1) problem transformation, in which the multi-label learning task is transformed into one or more single-label learning tasks; and 2) algorithm adaptation, in which specific learning algorithms are adapted to handle multi-label problems directly.

In this work we highlight a problem transformation method called Binary Relevance (BR) (LUACES et al., 2012). In this method, the multi-label problem is transformed into $c$ binary classifiers, one for each different label in $\mathscr{L}$. Then, a dataset $D_{\lambda_{j}}, 1 \leq j \leq c$, is created for each label $\lambda_{j} \in \mathscr{L}$ containing all instances of the original dataset. Each instance $\mathbf{x}_{i}$ in $D_{\lambda_{j}}$ is labeled positively if $\lambda_{j} \in L_{i}$ and negatively otherwise.

This method is appropriate for the problem addressed in this work due to three main reasons: 1) BR presents a competitive performance with respect to more complex methods (LUACES et al., 2012); 2) this is a popular, simple and intuitive method (MAIMON; ROKACH, 2010); and 3) after data transformation, any binary learning method can be taken as base learner (LUACES et al., 2012). This characteristic enables us to construct the multi-label model discussed in Section 5.2.5. 


\subsection{Summary}

In this chapter, we covered the state-of-the-art for the topics addressed in this thesis. First, we contextualized the software testing activities and pointed out the SDP models as an alternative to improve the management of testing resources. The CPDP research area has emerged in recent years when observed from the historical perspective of the general SDP literature. Even though many solutions were proposed aiming at improving the performance of CPDP models, this issue is still a challenge. In the next chapter we evaluate the feasibility of using feature selection methods to improve the performance of two CPDP instance filtering methods. In this experiment, we focus on the performance of specific transfer learning solutions. In the general context of CPDP, no extensive comparison of transfer learning solutions was conducted in the literature. In Chapter 4, we present a comparative analysis covering a representative sample of the state-of-the-art in CPDP. In this experiment, we provide a uniform experimental setup aiming at mitigating the conclusion instability, discussed in this chapter. Another factor related to the conclusion instability is the lack of an inductive bias suitable for all domains. This issue is investigated in the literature of meta-learning for algorithm recommendation, also discussed in this chapter. These concepts cover the necessary background for the understanding of the meta-learning solution proposed in Chapter 5. The proposed solution addresses several particularities inherent to the CPDP context, including the multi-label learning context, discussed in this chapter. 
CHAPTER

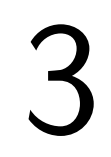

\section{FEATURE SUBSET SELECTION AND INSTANCE FILTERING FOR CPDP - CLASSIFICATION AND RANKING}

\subsection{Introduction}

In an ideal defect prediction scenario, it would be possible to predict the exact number of defects for each part of the software. However, this goal is hard to achieve or even impossible due to the lack of good quality data in practice (YANG; TANG; YAO, 2015). Thus, an alternative is to simplify the prediction goal and make it feasible for practical application. In the literature there are two main types of software defect prediction being studied: classification and ranking, as discussed in Section 2.2. In the first, the goal is to classify whether a software part is defective or not (OSTRAND; WEYUKER; BELL, 2004; ZIMMERMANN; NAGAPPAN, 2008; HERZIG et al., 2013). In practice, this kind of prediction allows to distribute the test resources more efficiently, although does not differentiate the level of importance between the defective parts (WEYUKER; OSTRAND; BELL, 2010). In the second type, the goal is to predict the instances more likely to contain the larger number of defects and rank them in a decreasing order (DENARO; PEZZè, 2002; WEYUKER; OSTRAND; BELL, 2010; YANG; TANG; YAO, 2015). In a context where resources are limited, this kind of prediction allows to direct the software quality assurance team to the most defective parts first (OSTRAND; WEYUKER; BELL, 2005). Both prediction goals have their own singularities and practical importance. An analysis involving the two prediction goals provides a comprehensive perspective in the current state-of-the-art of software defect prediction.

In another ideal context of defect prediction it would be possible to predict a software from a base of knowledge constructed from known external projects. This approach is 
investigated in the CPDP context, as discussed in Section 2.4. Among the CPDP solutions proposed in the literature, in this chapter, we highlight the filtering methods proposed by Turhan et al. (2009) and Peters, Menzies and Marcus (2013). These methods aim at building an accurate filtered dataset by selecting the most similar instances from the CPDP training set. Both methods make use of the Euclidean distance on measuring the similarity between instances. This similarity measure, though, originally considers the entire set of features available for a dataset.

We propose that using only the most relevant features on measuring the similarity between instances can improve the performance of the mentioned filtering methods. In order to select the most relevant features of a dataset we refer to the Feature Selection (FS) methods, widely investigated and adopted on the data mining literature (ZHAO et al., 2011). We evaluate four distinct FS methods: Information Gain (COVER; THOMAS, 1991), Relief (KONONENKO, 1994), CFS (ZHAO et al., 2011), and Sparcl (WITTEN; TIBSHIRANI, 2010). We also compare the use of two specific subset of features: code metrics (JURECZKO; MADEYSKI, 2010) and network metrics (ZIMMERMANN; NAGAPPAN, 2008). We call the evaluated methods as Instance Filtering methods based on Feature subset Selection (IFFS).

In this chapter we present an empirical evaluation of the mentioned IFFS methods for both prediction contexts - Classification and Ranking. The content presented in this chapter encompasses two publications of the authors. We proposed this experimental study in Porto and Simao (2016b) and extended it for both contexts - Classification and Ranking - in Porto and Simao (2016a). The two experimentation contexts are similar on their structure although differ in relation to the datasets, the prediction model, and the performance measures, as discussed below.

We aim at answering the following research questions for both prediction contexts:

RQ3.1 Can IFFS methods lead to better performances on CPDP models?

RQ3.2 Which IFFS methods present better performances on CPDP?

This chapter is organized as follows. In Section 3.2 we present the evaluated instance filtering methods. In Section 3.3 we present the feature selection concept applied for instance filtering methods. In Section 3.4 we present the experimental setup and methodology for the conducted experiments. In Section 3.5 we analyze the obtained results. In Section 3.6 we discuss the threats to validity. In Section 3.7 we summarize the obtained conclusions. 


\subsection{Instance Filtering Methods}

Given a cross-project training set and a test set to be predicted, the filtering methods aim at selecting only the most relevant training instances. The resulting filtered dataset may improve the efficiency obtained from CPDP models.

Turhan et al. (2009) proposed a filtering method based on the nearest neighbor filter. On this method, here called Burak filter, each test instance is compared with the entire training set. This comparison is based on the Euclidean distance and considers the entire set of independent variables. Then, for each test instance, the $k$ most similar training instances are selected to compose the filtered dataset. This method is illustrated in Figure 9(b), where for each test instance the two most similar training instances are selected. As illustrated, the same training instance can be similar with more than one test instance. The instance labeled with ' $\mathrm{L}$ ' is not included in the filtered dataset.

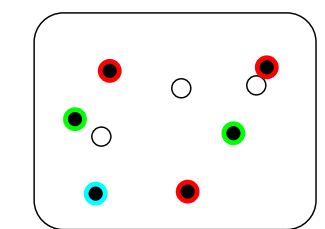

(a) Training + Test instances

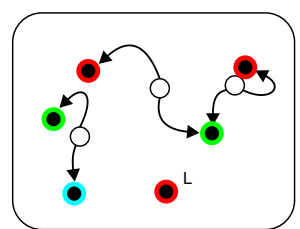

(b) Burak filter

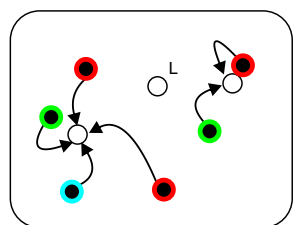

(c) Peters filter 1

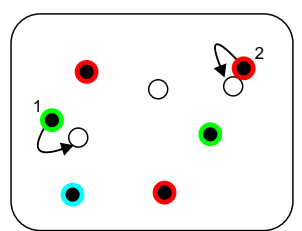

(d) Peters filter 2

Figure 9 - Illustration of the procedure executed for each filtering method evaluated. On Figure (a), the colored instances represent the cross-project training data and the white instances represent the test data. See text for more details (Adapted from (PETERS; MENZIES; MARCUS, 2013)).

The method proposed by Peters, Menzies and Marcus (2013) selects the most similar instances from a different perspective. First, the training instances are clustered considering all the test instances as centroids. The clustering process makes use of the K-means algorithm with Euclidean distance (JAIN, 2010). As a result, each training instance is associated with its most similar centroid (test instance). This first step is illustrated in Figure 9(c). Note that a test instance can be not selected (label 'L'). Second, for each test instance is selected the most similar training instance of the respective cluster. This second step is illustrated in Figure 9(d). The instances labeled with ' 1 ' and '2' represent the most similar instances and the resulting filtered dataset.

For both filtering methods, the resulting filtered dataset is then used as the training set.

\subsection{Feature Subset Selection}

Both instance filtering methods presented above make use of the Euclidean distance on measuring the similarity between instances. The set of independent variables for an instance $m$ can be represented by a vector $X_{m}=\left\{x_{1}^{m}, x_{2}^{m}, \ldots, x_{n}^{m}\right\}$, where $\left|X_{m}\right|=n$ is the 
number of variables (or features). The Euclidean distance between two instances $i$ and $j$ can be defined as

$$
d\left(X_{i}, X_{j}\right)=\sqrt{\sum_{k=1}^{n}\left(x_{k}^{i}-x_{k}^{j}\right)^{2}}
$$

Let $S$ be a subset of features composed of the most relevant features in $X$. We propose that using only the most relevant features on the Euclidean distance (i.e., $d\left(S_{i}, S_{j}\right)$ on Equation 3.1) can lead to a more accurate filtered dataset and then to a more efficient defect model. In order to select the most relevant features $S \subseteq X$, we evaluate different FS methods.

The FS methods have been widely investigated in the data mining literature (ZHAO et al., 2011). Traditionally, the FS methods are applied in high dimensional data in order to reduce the data dimensionality by removing the irrelevant and the redundant features (ZHAO et al., 2011). We focused on a specific category of FS methods called filter model since they are not dependent on the data mining algorithm. We evaluate three supervised methods: Information Gain (COVER; THOMAS, 1991), Relief (KONONENKO, 1994), and CFS (ZHAO et al., 2011); and one unsupervised method: Sparcl (WITTEN; TIBSHIRANI, 2010). The supervised methods select the most relevant features based on the target attribute. The unsupervised methods, however, select the most relevant features based on their characteristics only, independently of the target attribute.

These different methods represent part of the most important FS methods known in the literature. A specialized feature selection text can be found in Zhao et al. (2011) and Witten and Tibshirani (2010).

\subsection{Experimental Setup}

We conducted an in silico experimentation in order to evaluate the performance of IFFS methods in both contexts: Classification and Ranking. For each experimentation context, we evaluate two filtering methods - Burak Filter (TURHAN et al., 2009) and Peters Filter (PETERS; MENZIES; MARCUS, 2013) - combined with feature subset selection. We analyzed either Feature Selection (FS) methods and specific Software metric Subsets (SS). All the experiments and data analysis were implemented and conducted with $R^{1}$.

$1<$ https://www.r-project.org/> 


\subsubsection{Data Collection}

\section{Software Projects}

The experiments in this chapter were conducted based on 36 versions of 11 Java open source projects, available in the PROMISE repository ${ }^{2}$. The collection and preparation of defect data were made and provided by Jureczko and Madeyski (2010). The authors provide a $\operatorname{link}^{3}$ with detailed information about the software projects and the construction of each dataset. Each instance in a dataset represents a Java object-oriented class (OO class). Originally, the dependent variable corresponds to the number of defects found for each OO class.

For the ranking analysis we considered the datasets in their original forms. Table 3 presents the distribution and density of defects for each dataset. The ranking characteristics and defect distribution presented in this table are better discussed in Section 3.4.5. For the classification analysis we converted the dependent variable to a binary classification problem: 1, for number of defects $>0$; or 0 , otherwise. Table 4 lists the number of instances, number of defective classes, and defect rate for each of the analyzed datasets.

We evaluate two distinct sets of metrics as the independent variables: code metrics (CODE) and network metrics (NET). These metrics are numerical and can be automatically extracted directly from the source code files.

\subsubsection{Code Metrics}

The code metrics set is composed of 20 metrics and include complexity metrics, C\&K (Chidamber and Kemerer) metrics, and structural code metrics. This set of metrics is briefly described in Table 5 . This metric set has been reported as good quality indicators on literature, as discussed in Jureczko and Madeyski (2010). These metrics can be extracted by using the $\mathrm{Ckjm}^{4}$ tool. Further details can be found in Jureczko and Madeyski (2010).

\subsubsection{Network Metrics}

The theory of social network analysis combines concepts of graph theory with statistical mechanisms to describe the topological properties of social networks. The nodes represent the network elements (e.g. actors, humans) and the edges (ties or links) represent their interactions (or relationships). In the context of this work, each node represents a software file and each link represents a dependency between two files. The characterization of softwares by network metrics provides a different perspective for SDP models. These metrics capture the properties of the interactions between elements while

\footnotetext{
$<$ http://openscience.us/repo/>

$<$ http://purl.org/MarianJureczko/MetricsRepo/>

$<$ http://gromit.iiar.pwr.wroc.pl/p_inf/ckjm>
} 
Table 3 - Summary of the projects characteristics for Ranking. We analyzed a ranking performance measure that measures the percentage of defects $(\alpha)$ in the former $(\beta)$ instances of the ranking. Usually, in the literature, $\beta=20 \%$. In this study we fixed $\alpha \approx 80 \%$ and the $\beta$ varies depending on the defect distribution for each project.

\begin{tabular}{|c|c|c|c|c|c|c|}
\hline Project & No. of Classes & Sum of Defects & $\% \beta$ & $\% \alpha$ & No. of Classes in $\beta$ & No. of Defects in $\alpha$ \\
\hline ant-1.3 & 125 & 33 & 0.11 & 0.82 & 14 & 27 \\
\hline ant-1.4 & 177 & 46 & 0.17 & 0.8 & 30 & 37 \\
\hline ant-1.5 & 291 & 35 & 0.09 & 0.8 & 25 & 28 \\
\hline ant-1.6 & 348 & 184 & 0.16 & 0.8 & 56 & 148 \\
\hline ant-1.7 & 493 & 284 & 0.17 & 0.8 & 83 & 228 \\
\hline forrest-0.6 & 6 & 1 & 0.17 & 1 & 1 & 1 \\
\hline forrest- 0.7 & 29 & 15 & 0.07 & 0.73 & 2 & 11 \\
\hline forrest-0.8 & 32 & 6 & 0.06 & 1 & 2 & 6 \\
\hline ivy-1.1 & 111 & 233 & 0.27 & 0.81 & 30 & 188 \\
\hline ivy-1.4 & 239 & 18 & 0.05 & 0.83 & 13 & 15 \\
\hline ivy-2.0 & 351 & 56 & 0.08 & 0.8 & 29 & 45 \\
\hline $\log 4 \mathrm{j}-1.0$ & 82 & 57 & 0.23 & 0.81 & 19 & 46 \\
\hline $\log 4 \mathrm{j}-1.1$ & 76 & 79 & 0.24 & 0.82 & 18 & 65 \\
\hline $\log 4 \mathrm{j}-1.2$ & 123 & 355 & 0.66 & 0.8 & 81 & 285 \\
\hline lucene-2.0 & 195 & 268 & 0.23 & 0.8 & 44 & 215 \\
\hline lucene- 2.2 & 245 & 412 & 0.29 & 0.8 & 72 & 330 \\
\hline lucene-2.4 & 337 & 629 & 0.31 & 0.8 & 105 & 505 \\
\hline pbeans-1.0 & 26 & 36 & 0.5 & 0.81 & 13 & 29 \\
\hline pbeans-2.0 & 51 & 19 & 0.14 & 0.84 & 7 & 16 \\
\hline poi-1.5 & 222 & 326 & 0.3 & 0.8 & 66 & 261 \\
\hline poi-2.0 & 291 & 38 & 0.1 & 0.82 & 29 & 31 \\
\hline poi-2.5 & 360 & 454 & 0.4 & 0.8 & 144 & 365 \\
\hline poi-3.0 & 410 & 479 & 0.4 & 0.8 & 165 & 384 \\
\hline synapse-1.0 & 155 & 21 & 0.08 & 0.81 & 12 & 17 \\
\hline synapse-1.1 & 220 & 99 & 0.19 & 0.81 & 41 & 80 \\
\hline synapse- 1.2 & 251 & 145 & 0.23 & 0.8 & 57 & 116 \\
\hline velocity-1.4 & 182 & 196 & 0.52 & 0.8 & 95 & 157 \\
\hline velocity-1.5 & 203 & 325 & 0.36 & 0.8 & 74 & 260 \\
\hline velocity-1.6 & 216 & 188 & 0.19 & 0.81 & 42 & 152 \\
\hline xalan- 2.4 & 409 & 119 & 0.14 & 0.81 & 56 & 96 \\
\hline xalan-2.5 & 714 & 502 & 0.36 & 0.8 & 258 & 402 \\
\hline xalan-2.6 & 773 & 525 & 0.29 & 0.8 & 221 & 420 \\
\hline xalan-2.7 & 763 & 952 & 0.74 & 0.8 & 565 & 762 \\
\hline xerces-1.2 & 377 & 115 & 0.13 & 0.8 & 48 & 92 \\
\hline xerces-1.3 & 391 & 193 & 0.09 & 0.81 & 37 & 156 \\
\hline xerces-1.4 & 269 & 555 & 0.33 & 0.8 & 88 & 445 \\
\hline Mean & 265.08 & 222.17 & 0.25 & 0.82 & 73.39 & 178.36 \\
\hline
\end{tabular}

the code metrics, for example, capture the properties of a file in an individual perspective. The first study in this context, applying network metrics for SDP, was conducted by Zimmermann and Nagappan (2008). Subsequently, their study was replicated in (TOSUN; TURHAN; BENER, 2009), (PRATEEK; PASALA; ARACENA, 2013), and (HERZIG et al., 2013).

The network metrics set was extracted in two steps. First, it was constructed the dependency graph for each analyzed software version. Each OO class file is represented by a vertex and any dependency relationship between two vertices is represented by an edge on the graph. This process was made by using the PF-CDA tool ${ }^{5}$.

Once created the dependency graph, we extracted the network metrics data. Table 6 briefly describes the metrics analyzed in this study. This set of metrics can be grouped in two categories: the ego metrics and the global metrics. The ego metrics refer to the

$5 \quad<$ http://www.dependency-analyzer.org/> 
Table 4 - Summary of the projects characteristics for Classification.

\begin{tabular}{|c|c|c|c|}
\hline Project & No. of Classes & No. of Defective Classes & \% of Defective Classes \\
\hline ant-1.3 & 125 & 20 & 0.16 \\
\hline ant-1.4 & 177 & 39 & 0.22 \\
\hline ant-1.5 & 292 & 32 & 0.11 \\
\hline ant-1.6 & 349 & 92 & 0.26 \\
\hline ant-1.7 & 493 & 139 & 0.28 \\
\hline forrest- 0.6 & 6 & 1 & 0.17 \\
\hline forrest- 0.7 & 29 & 5 & 0.17 \\
\hline forrest- 0.8 & 32 & 2 & 0.06 \\
\hline ivy-1.1 & 111 & 63 & 0.57 \\
\hline ivy-1.4 & 239 & 16 & 0.07 \\
\hline ivy-2.0 & 351 & 40 & 0.11 \\
\hline $\log 4 \mathrm{j}-1.0$ & 82 & 30 & 0.37 \\
\hline $\log 4 \mathrm{j}-1.1$ & 76 & 31 & 0.41 \\
\hline $\log 4 \mathrm{j}-1.2$ & 123 & 117 & 0.95 \\
\hline lucene-2.0 & 195 & 91 & 0.47 \\
\hline lucene-2.2 & 245 & 142 & 0.58 \\
\hline lucene-2.4 & 337 & 200 & 0.59 \\
\hline pbeans-1.0 & 26 & 20 & 0.77 \\
\hline pbeans- 2.0 & 51 & 10 & 0.2 \\
\hline poi-1.5 & 222 & 131 & 0.59 \\
\hline poi-2.0 & 293 & 36 & 0.12 \\
\hline poi- 2.5 & 360 & 223 & 0.62 \\
\hline poi-3.0 & 413 & 260 & 0.63 \\
\hline synapse-1.0 & 155 & 16 & 0.1 \\
\hline synapse-1.1 & 220 & 60 & 0.27 \\
\hline synapse-1.2 & 252 & 86 & 0.34 \\
\hline velocity-1.4 & 186 & 138 & 0.74 \\
\hline velocity-1.5 & 204 & 137 & 0.67 \\
\hline velocity-1.6 & 217 & 76 & 0.35 \\
\hline xalan-2.4 & 409 & 79 & 0.19 \\
\hline xalan-2.5 & 717 & 359 & 0.5 \\
\hline xalan-2.6 & 776 & 328 & 0.42 \\
\hline xalan-2.7 & 762 & 754 & 0.99 \\
\hline xerces-1.2 & 385 & 71 & 0.18 \\
\hline xerces-1.3 & 398 & 69 & 0.17 \\
\hline xerces-1.4 & 275 & 168 & 0.61 \\
\hline Mean & 266.19 & 113.36 & 0.39 \\
\hline
\end{tabular}

metrics extracted from the ego network. Given a vertex $v$, its ego network contains all vertices directly connected to $v$ and their respective connections. The global metrics refer to the metrics extracted from the original entire graph. In addition, some metrics can be extracted from three different kinds of networks according to the direction of edges: incoming edges (In), outgoing edges (Out), or undirected edges (All). We analyzed 24 distinct network metrics. From these 24 metrics, considering all their variations (In, Out, All), we extracted a total of 54 network metrics. We computed these metrics by using the igraph package (CSARDI; NEPUSZ, 2006). The analyzed metrics were selected according to availability at igraph package or feasibility to implement.

\subsubsection{Feature Subset Selection}

We analyzed the FS methods: CFS (FS_CFS) (ZHAO et al., 2011), Information Gain (FS_IG) (COVER; THOMAS, 1991), Relief (FS_RLF) (KONONENKO, 1994), and Sparcl (FS_SPC) (WITTEN; TIBSHIRANI, 2010). The first three are supervised methods. Thus, the feature selection is performed from the training data, in which the 
Table 5 - Code metrics (Descriptions adapted from (JURECZKO; MADEYSKI, 2010)).

\begin{tabular}{|c|c|}
\hline Name & Description \\
\hline $\begin{array}{l}\text { Average Method } \\
\text { Complexity (AMC) }\end{array}$ & $\begin{array}{l}\text { This metric measures the average method size for each class. Size of a method is equal to the } \\
\text { number of Java binary codes in the method. }\end{array}$ \\
\hline $\begin{array}{l}\text { Cohesion Among Class } \\
\text { Methods (CAM) }\end{array}$ & $\begin{array}{l}\text { This metric computes the relatedness among methods of a class based upon the parameter list } \\
\text { of the methods. The metric is computed using the summation of number of different types of } \\
\text { method parameters in every method divided by a multiplication of number of different method } \\
\text { parameter types in whole class and number of methods. }\end{array}$ \\
\hline $\begin{array}{l}\text { Afferent couplings } \\
\text { (Ca) }\end{array}$ & The Ca metric represents the number of classes that depend upon the measured class. \\
\hline $\begin{array}{l}\text { Efferent couplings } \\
\text { (Ce) }\end{array}$ & The Ce metric represents the number of classes that the measured class is depended upon. \\
\hline $\begin{array}{l}\text { Coupling Between } \\
\text { Methods (CBM) }\end{array}$ & $\begin{array}{l}\text { The metric measures the total number of new/redefined methods to which all the inherited } \\
\text { methods are coupled. There is a coupling when at least one of the conditions given in the IC } \\
\text { metric is held. }\end{array}$ \\
\hline $\begin{array}{l}\text { Coupling between } \\
\text { object classes }(\mathrm{CBO})\end{array}$ & $\begin{array}{l}\text { The CBO metric represents the number of classes coupled to a given class (efferent couplings } \\
\text { and afferent couplings). }\end{array}$ \\
\hline $\begin{array}{l}\text { Cyclomatic } \\
\text { Complexity (CC) }\end{array}$ & $\begin{array}{l}\mathrm{CC} \text { is equal to number of different paths in a method (function) plus one. The McCabe cyclomatic } \\
\text { complexity is defined as: } \mathrm{CC}=\mathrm{E}-\mathrm{N}+\mathrm{P} \text {; where } \mathrm{E} \text { is the number of edges of the graph, } \mathrm{N} \text { is the } \\
\text { number of nodes of the graph, and } \mathrm{P} \text { is the number of connected components. } \mathrm{CC} \text { is the only } \\
\text { method size metric. The constructed models make the class size predictions. Therefore, the metric } \\
\text { had to be converted to a class size metric. Two metrics has been derived: }\end{array}$ \\
\hline
\end{tabular}

- MAX_CC - the greatest value of CC among methods of the investigated class;

- AVG_CC - the arithmetic mean of the $\mathrm{CC}$ value in the investigated class.

\begin{tabular}{ll}
\hline $\begin{array}{l}\text { Data Access Metric } \\
\text { (DAM) }\end{array}$ & $\begin{array}{l}\text { This metric is the ratio of the number of private (protected) attributes to the total number of } \\
\text { attributes declared in the class. }\end{array}$ \\
\hline $\begin{array}{l}\text { Depth of Inheritance } \\
\text { Tree (DIT) }\end{array}$ & $\begin{array}{l}\text { The DIT metric provides for each class a measure of the inheritance levels from the object } \\
\text { hierarchy top. }\end{array}$ \\
\hline $\begin{array}{l}\text { Inheritance Coupling } \\
\text { (IC) }\end{array}$ & $\begin{array}{l}\text { This metric provides the number of parent classes to which a given class is coupled. A class is } \\
\text { coupled to its parent class if one of its inherited methods functionally dependent on the new } \\
\text { or redefined methods in the class. A class is coupled to its parent class if one of the following } \\
\text { conditions is satisfied: }\end{array}$
\end{tabular}
conditions is satisfied:

- One of its inherited methods uses an attribute that is defined in a new/redefined method;

- One of its inherited methods calls a redefined method;

- One of its inherited methods is called by a redefined method and uses a parameter that is defined in the redefined method.

\begin{tabular}{|c|c|}
\hline $\begin{array}{l}\text { Lack of cohesion in } \\
\text { methods (LCOM) }\end{array}$ & $\begin{array}{l}\text { The LCOM metric counts the sets of methods in a class that are not related through the sharing } \\
\text { of some of the class fields. }\end{array}$ \\
\hline $\begin{array}{l}\text { Lack of cohesion in } \\
\text { methods (LCOM3) }\end{array}$ & $\begin{array}{ll}\text { LCOM3 }=\frac{\left(\frac{1}{a} \sum_{j=1}^{a} \mu\left(A_{j}\right)\right)-m}{1-m} & \bullet \mathrm{m}-\text { number of methods in a class; } \\
& \bullet \mu(A) \text { - number of methods that access the attribute A. }\end{array}$ \\
\hline Lines of Code (LOC) & $\begin{array}{l}\text { The LOC metric calculates the number of lines of code in the Java binary code of the class under } \\
\text { investigation. }\end{array}$ \\
\hline $\begin{array}{l}\text { Measure of Functional } \\
\text { Abstraction (MFA) }\end{array}$ & $\begin{array}{l}\text { This metric is the ratio of the number of methods inherited by a class to the total number of } \\
\text { methods accessible by the member methods of the class. }\end{array}$ \\
\hline $\begin{array}{l}\text { Measure of } \\
\text { Aggregation (MOA) }\end{array}$ & $\begin{array}{l}\text { This metric measures the extent of the part-whole relationship, realized by using attributes. The } \\
\text { metric is a count of the number of class fields whose types are user defined classes. }\end{array}$ \\
\hline $\begin{array}{l}\text { Number of Children } \\
\text { (NOC) }\end{array}$ & The NOC metric simply measures the number of immediate descendants of the class. \\
\hline $\begin{array}{l}\text { Number of Public } \\
\text { Methods (NPM) }\end{array}$ & The NPM metric counts all the methods in a class that are declared as public. \\
\hline $\begin{array}{l}\text { Response for a Class } \\
\text { (RFC) }\end{array}$ & $\begin{array}{l}\text { The RFC metric measures the number of different methods that can be executed when an object } \\
\text { of that class receives a message. }\end{array}$ \\
\hline $\begin{array}{l}\text { Weighted methods per } \\
\text { class (WMC) }\end{array}$ & $\begin{array}{l}\text { The value of the WMC is equal to the number of methods in the class (assuming unity weights } \\
\text { for all methods). }\end{array}$ \\
\hline
\end{tabular}


Table 6 - The analyzed network metrics. The metrics were computed for incoming (In), outgoing (Out), and/or undirected (All) dependencies (Descriptions adapted from (ZIMMERMANN; NAGAPPAN, 2008) and (HERZIG et al., 2013)).

\begin{tabular}{|c|c|}
\hline \multicolumn{2}{|r|}{ Ego Network Metrics } \\
\hline Metric Name & Description \\
\hline Size ${ }^{\text {In Out All }}$ & \# nodes connected to the ego network \\
\hline Ties $^{\text {In Out All }}$ & \# directed ties corresponds to the number of edges \\
\hline Pairs ${ }^{\text {In Out All }}$ & \# ordered pairs is the maximal number of directed ties \\
\hline Density $^{\text {In Out All }}$ & $\%$ of possible ties that are actually present \\
\hline WeakComp $^{\text {In Out All }}$ & \# weak components in neighborhood \\
\hline nWeakComp ${ }^{\text {In Out All }}$ & \# weak components normalized by size \\
\hline TwoStepReach ${ }^{\text {In Out All }}$ & $\%$ nodes that are two steps away \\
\hline ReachEfficiency $^{\text {In Out All }}$ & TwoStepReach normalized by size \\
\hline EgoBetween ${ }^{\text {In Out All }}$ & \% shortest paths between neighbors through ego \\
\hline nEgoBetween $^{\text {In Out All }}$ & EgoBetween normalized by the size \\
\hline EffSize $^{A l l}$ & size of the ego network minus the average number of ties between their nodes \\
\hline \multicolumn{2}{|r|}{ Global Network Metrics } \\
\hline Metric Name & Description \\
\hline Degree ${ }^{\text {In Out All }}$ & \# dependencies for a node \\
\hline Closeness $^{\text {In Out All }}$ & Total length of the shortest paths from a node (or to a node) to all other nodes \\
\hline Reach $^{\text {In Out All }}$ & How far a node can receive or send information in the network \\
\hline Spread $^{\text {In Out All }}$ & How wide a node can receive or send information in the network \\
\hline Radiality $^{\text {In Out All }}$ & Radial centrality of a node \\
\hline Eigenvector $^{A l l}$ & Measure the influence of an node in a network \\
\hline Betweenness $^{\text {All }}$ & Measure for a node in how many shortest paths between other nodes it occurs \\
\hline Power ${ }^{A l l}$ & Bonacich Power centrality measure \\
\hline Clustering ${ }^{\text {All }}$ & $\%$ of adjacent vertices to a vertex be connected \\
\hline Page Rank ${ }^{A l l}$ & Google's PageRank value. Generalization of Eigenvector metric \\
\hline Eccentricity $^{A l l}$ & Distance of the shortest path from a vertex to the farthest vertex on the network \\
\hline $\mathrm{Hub}^{\text {All }}$ & Kleinberg's Hub measure. Generalization of Eigenvector metric \\
\hline Authority ${ }^{\text {All }}$ & Kleinberg's Authority measure. Generalization of Eigenvector metric \\
\hline
\end{tabular}

target attribute is known. The Sparcl method is unsupervised. Thus, can be performed from both training (FS_SPC_Tr) and test data (FS_SPC_Te) since no target attribute information is required. The methods FS_IG, FS_RLF, and FS_SPC provides a rank of weights that measures the relevance of each feature, according to some criterion. Deciding the best $k$ features to compose the feature subset is not a trivial task (ZHAO et al., 2011). Thus, for each method we analyzed four different values of $k=\{5,10,15,20\}$ in order to approximate the best $k$ configuration. Considering all methods and their variations of $k$, we analyzed 17 FS methods for each filtering method. We used the $\mathrm{R}$ packages 'FSelector' (ROMANSKI; KOTTHOFF, 2014) and 'sparcl' (WITTEN; TIBSHIRANI, 2010) with the implemented FS methods. All the analyzed FS methods and their respective implementations permit the application of both types of datasets with continuous (for ranking) and discrete (for classification) values in the dependent variable.

In addition, we investigated two distinct metric subsets: code metrics (SS_CODE) and network metrics (SS_NET). For each metric subset, we applied a Pearson correlation test in order to disregard the metrics considered redundant, with correlation greater than 0.90 (TORGO, 2010). We also analyzed the performance with the original set of features (Orig), as a comparative reference. 


\subsubsection{Experiment Design}

The experiment design is presented in Figure 10. The structure is quite similar for both experimentation contexts - Classification and Ranking. The differences are concentrated in three points: the dataset (Section 3.4.1); the prediction model (Section 3.4.4); and the performance measures (Section 3.4.5), as discussed individually in the text.

For this experiment, we follow a variation of the leave-one-out cross-validation procedure (DORES et al., 2016), here called Cross-Project Leave-One-Out (CPLOO). In this variation, the training set contains all the available project versions except the versions of the same project of the current test set, as described below.

First, we joined all datasets in one unique Cross-Project Dataset (CPD). Then, we conducted a many-to-one cross-project analysis (GUNARATHNA; TURHAN; HOSSEINI, 2016). Consider a project $\mathscr{P}$, the set $\mathscr{V}_{\mathscr{P}}$ of all versions of $\mathscr{P}$, and a specific version $v_{i}$ of $\mathscr{V}_{\mathscr{P}}$. For each $v_{i} \in \mathscr{V}_{\mathscr{P}}, 1 \leq i \leq\left|\mathscr{V}_{\mathscr{P}}\right|$, we used $v_{i}$ as test and CPD $\backslash \mathscr{V}_{\mathscr{P}}\left(\mathscr{V}_{\mathscr{P}}\right.$ subtracted from $\mathrm{CPD})$ as training. In this way, we can analyze the predictive performance disregarding any bias from the different versions of a same project.

The training data is filtered by applying the Burak and Peters filters combined with the studied feature subsets presented above. The filtered training data is then used to construct the defect prediction models.

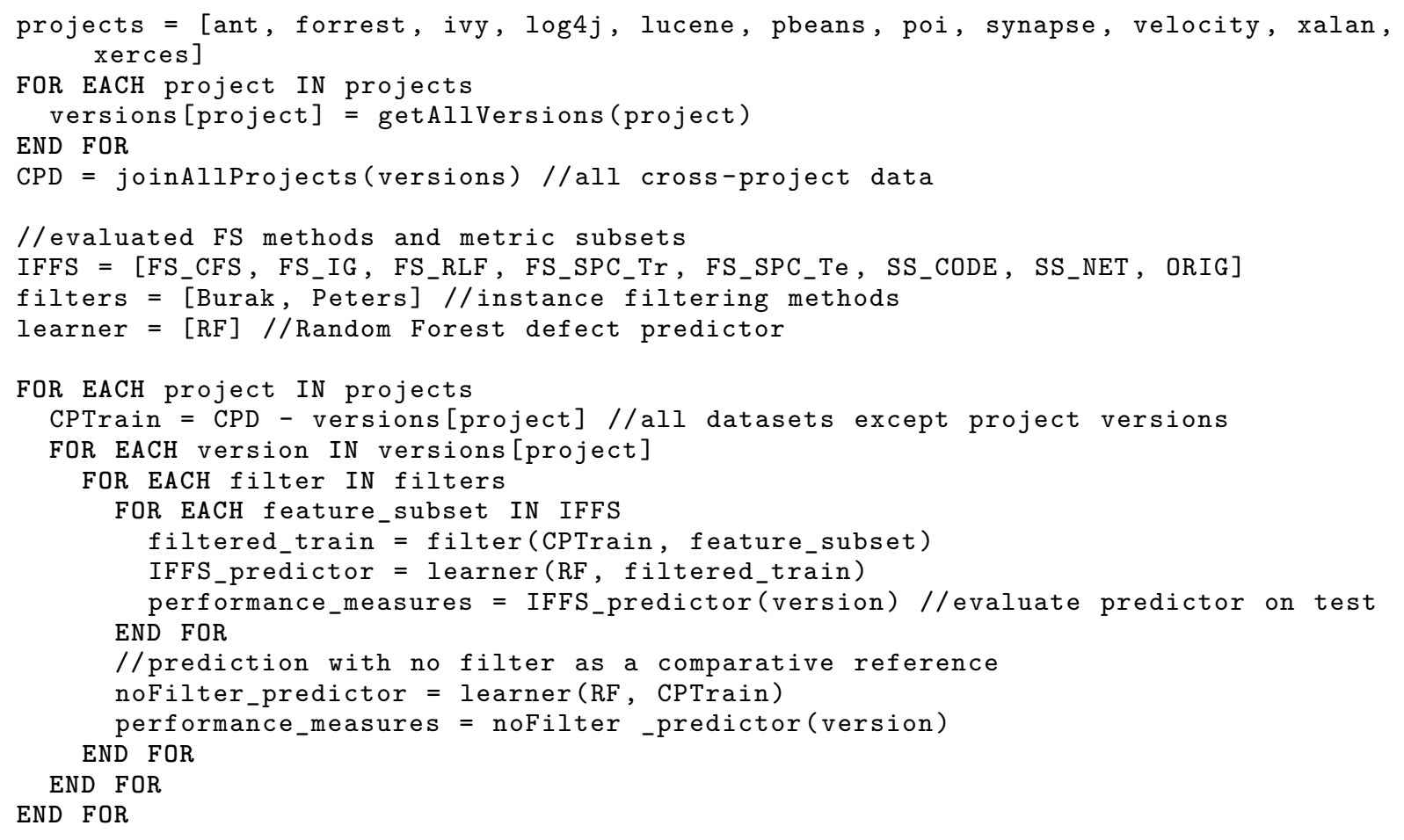

Figure 10 - Pseudocode with the experiment design for both Classification and Ranking. 


\subsubsection{Prediction Models}

The models were constructed by using the Random Forest Algorithm (BREIMAN, 2001). This algorithm has shown good performances compared with others algorithms in both contexts - Classification (LESSMANN et al., 2008; JIANG; CUKIC; MA, 2008) and Ranking (WEYUKER; OSTRAND; BELL, 2010; YANG; TANG; YAO, 2015). Lessmann et al. (2008) argue that Random Forest is the current state-of-the-art defect predictor. Random Forest is an ensemble of models based on decision trees. At the training time it is constructed a multitude of decision trees from which is calculated and returned the mode of the classes (for classification) or the mean prediction (for regression) of the individual trees (BREIMAN, 2001). A better description of the Random Forest algorithm is presented in Section 2.3.5.

Differently of the classification models, the ranking models are constructed in two steps: first, it is constructed a regression model and then the instances are ranked according to their predicted number of defects. For the experiments, we used the $\mathrm{R}$ package 'randomForest' (LIAW; WIENER, 2002). It is important to note that some internal processes of Random Forest, such as sampling and bagging, includes randomness which can lead to different models in each execution. Thus, we constructed 30 models for each evaluated method. The results are presented considering the performance mean.

In Zimmermann and Nagappan (2008) and Herzig et al. (2013) the authors argue that network metrics can lead to better prediction performances when combined with code metrics. Thus, we considered both metric sets CODE and NET as predictors on experiments. Again, the features with high Pearson correlation $(>0.90)$ were disregarded (HERZIG et al., 2013). The remaining set with non-redundant features is composed of 20 code metrics and 35 network metrics.

\subsubsection{Performance Measures}

\section{Classification}

We analyzed three performance measures for classification: recall (pd), probability of false alarm (pf), and g-measure (JAPKOWICZ; SHAH, 2011). Both measures recall and pf have the focus on the defective class and are efficient for evaluating datasets with a small number of defective examples (MENZIES et al., 2007). These metrics were also used to evaluate the performance of the two analyzed filters in their original works (TURHAN et al., 2009; PETERS; MENZIES; MARCUS, 2013), which contributed for their use in this study. Table 7 presents the confusion matrix and the analyzed measures.

The recall measures how much of the actual defects were found. The $p f$ can be described as the probability of an instance predicted as defective do not be a real defective instance. The optimal pf is $0 \%$ and the worst result is $100 \%$. The $g$-measure is the harmonic 
mean between recall and 1-pf (the specificity).

Table 7 - Performance measures for Classification.

(a) Confusion matrix

\begin{tabular}{cccc}
\cline { 3 - 4 } & \multicolumn{2}{c}{ Actual } \\
\cline { 2 - 4 } & & yes & no \\
\hline \multirow{2}{*}{ Predicted } & yes & TP & FP \\
\cline { 2 - 4 } & no & FN & TN \\
\hline
\end{tabular}

(b) Analyzed measures

\begin{tabular}{cc}
\hline recall $(\mathrm{pd})$ & $\frac{T P}{F N+T P}$ \\
\hline pf & $\frac{F P}{T N+F P}$ \\
\hline g-measure & $\frac{2 * p d *(1-p f)}{p d+(1-p f)}$ \\
\hline
\end{tabular}

\section{Ranking}

For the ranking task, we analyzed two performance measures: the percentage of defects $(\alpha)$ in the former $(\beta)$ instances of the ranking (OSTRAND; WEYUKER; BELL, 2005; WEYUKER; OSTRAND; BELL, 2010); and the Fault-Percentile-Average (FPA) (WEYUKER; OSTRAND; BELL, 2010; YANG; TANG; YAO, 2015).

The first measure is widely used in the literature since it is practical and simple. Weyuker, Ostrand and Bell (2010) studied datasets in which 20\% ( $\beta=20 \%$ ) of the most defective instances contains $80 \%(\alpha=80 \%)$ or more of the reported defects. This information can be used to analyze how many defects are present in the first $20 \%$ instances of the predicted ranking, in relation to the $80 \%$ already known. However, this cutoff $\beta=20 \%$ can varies for each project. In our study, for each project, we fixed $\alpha \approx 80 \%$ with $\beta$ ranging between $5 \%$ and $74 \%$, as we can see in Table 3 .

Although the first measure can be efficient for some purposes, it disregards the ordering of the remaining instances in the ranking. Furthermore, it is sensitive to the arbitrary cutoff. Weyuker, Ostrand and Bell (2010) proposed a general measure, called FPA, that takes into account the whole ranking performance. Consider $K$ instances $i_{1}, i_{2}, \ldots, i_{K}$, listed in an increasing order of predicted number of defects, being $i_{K}$ the most defective instance. Let $n_{k}$ be the actual number of defects for the instance $k$, and $N=n_{1}+\ldots+n_{K}$ be the total number of defects in the entire list of instances. For any $m$ in $1, \ldots, K$, the proportion of actual defects in the top $m$ predicted files is

$$
P_{m}=\frac{1}{N} \sum_{k=K-m+1}^{K} n_{k} .
$$

The FPA measure is then the average of the $P_{m}$ (WEYUKER; OSTRAND; BELL, 2010). It is defined as:

$$
F P A=\frac{1}{K} \sum_{m=1}^{K} P_{m} .
$$

In other words, FPA is the average of the percentage of actual defects contained in the top $m$ instances $(m=1,2, \ldots, K)$. It means that, if the prediction is good, the most 
defective instances will occur at or near to the top and then will be counted for most of the terms that contribute with the average.

\subsection{Results}

In this section we discuss the results for the respective research questions in both contexts - Classification and Ranking. We compare the performance of the evaluated IFFS methods with the performance of noFilter. In order to verify the statistical difference of performance, we use the non-parametric statistical test Wilcoxon's Signed-Rank ( $p$-value $<0.05)$. The symbols $\ominus$ and $\oplus$ represent, respectively, lower and higher performance accordingly to the mean value, with statistical significance. The absence of these symbols means no statistical difference between the compared performances.

\section{RQ3.1: Can IFFS methods lead to better performances on CPDP models?}

Classification

Table 8 shows the prediction performances obtained for each project. The performance on the noFilter column are reported by the g-measure $(g)$. All other columns are reported by the relative value $\Delta g=g-g_{\text {noFilter }}$, i.e., negative values show lower performances and positive values show higher performances in relation to noFilter. For a better visualization, we multiply the values by 100 . The bold values indicate the highest $\Delta g$ obtained for the respective project row. The projects are presented in decreasing order considering the higher $\Delta g$ value. The last row presents the mean value for each column. The columns Best FS present the best performance among all the evaluated FS methods. The columns NET and CODE present the performance obtained by considering the feature subsets SS_NET and SS_CODE.

We can analyze this table in different perspectives. First, we can notice that the columns Best FS presented better performance than Orig in all cases for both filtering methods. It means that at least one of the evaluated FS methods performs better than the original method. The FS methods presented the highest performances in most projects, as highlighted by the bold values. These improvements in performance can be crucial to define if a prediction model is appropriate or not for practical use. Zimmermann et al. (2009) presented a large experiment on cross-prediction feasibility. Their results show that only $3.4 \%$ of the studied models presented a prediction performance considered appropriate for practical use. They considered a successful defect model when accuracy, precision, and recall present performance greater than 75\%. Peters, Menzies and Marcus (2013) considered both performance thresholds of $60 \%$ and $75 \%$ for the g-measure. In Figure 11 we compare the percentage of projects with performance greater than $60 \%$ and $75 \%$ 
Table 8 - Performance obtained for the analyzed IFFS methods for both Burak and Peters Filters for Classification. All the performances are presented in relation to noFilter. Positive values mean better performances and negative values mean lower performances. The bold values indicate the highest performance for a project row. The symbols ${ }^{\oplus}$ and ${ }^{\ominus}$ represent the columns with significant difference in relation to noFilter.

\begin{tabular}{|c|c|c|c|c|c|c|c|c|c|}
\hline \multirow{2}{*}{ Project } & \multirow[b]{2}{*}{ noFilter (g) } & \multicolumn{4}{|c|}{ Burak Filter $(\Delta g)$} & \multicolumn{4}{|c|}{ Peters Filter $(\Delta g)$} \\
\hline & & Orig & NET & CODE & Best FS & Orig & NET & CODE & Best FS \\
\hline forrest- 0.6 & 0 & 0 & 0 & 0 & 94.29 & 0 & 8.33 & 58.07 & 58.52 \\
\hline velocity-1.4 & 7.84 & 6.49 & 2.1 & -7.58 & 49.78 & -1.74 & -0.73 & 11.75 & 45.5 \\
\hline xerces-1.4 & 33.27 & -2.6 & -5.61 & 3.88 & 45.15 & 20.97 & 25.28 & 15.31 & 38.34 \\
\hline velocity-1.5 & 9.21 & 7.15 & 6.61 & 0.43 & 27.13 & -2.22 & 5.85 & 10.49 & 41.34 \\
\hline $\log 4 \mathrm{j}-1.1$ & 45.49 & 4.37 & 31.98 & 25.79 & 39.86 & 11.81 & 16.91 & 22.89 & 30.8 \\
\hline pbeans-1.0 & 33.96 & -0.42 & 12.96 & -10.41 & 20.38 & 0 & 0 & 15.85 & 33.68 \\
\hline lucene-2.4 & 15.55 & -4.57 & -4.09 & 10.85 & 10.56 & 3.9 & -0.53 & 24.79 & 31.49 \\
\hline $\log 4 \mathrm{j}-1.0$ & 43.61 & 21.68 & 20.39 & 8.39 & 30.64 & 24.14 & 18.31 & 18.91 & 27.84 \\
\hline velocity-1.6 & 18.37 & -6.3 & -2.43 & -8.12 & 17.26 & -0.92 & 0.18 & 13.64 & 28.99 \\
\hline $\log 4 \mathrm{j}-1.2$ & 26.12 & -4.36 & -4.25 & 3.45 & 2.56 & -18.23 & -17.01 & 5.56 & 28.4 \\
\hline poi- 2.5 & 15.26 & 7.58 & 0.44 & 19.99 & 16.93 & 17.95 & -0.05 & 17.93 & 27.58 \\
\hline poi-3.0 & 26.12 & 3.42 & 5.42 & 4.77 & 12.45 & 12.03 & -9.85 & 16.29 & 22.55 \\
\hline lucene-2.2 & 31.38 & -18.4 & -3.31 & -0.75 & 5.16 & -4.41 & 20.09 & 13.47 & 19.74 \\
\hline xerces-1.3 & 46.53 & -3.15 & -5.26 & 6.48 & 19.21 & 4.21 & 15.9 & 14.23 & 19.97 \\
\hline synapse-1.1 & 35.81 & -0.41 & -20.41 & -13.93 & 11.51 & -11.9 & 2.78 & 6.25 & 17.57 \\
\hline synapse-1.2 & 45.3 & 2.79 & 3.07 & -3 & 13.61 & -5.54 & -32.53 & 6.89 & 11.04 \\
\hline xerces-1.2 & 36.68 & -3.07 & -3.76 & 3.65 & 13.5 & 1.39 & 8.3 & 6.78 & 11.82 \\
\hline synapse-1.0 & 56 & -3.27 & -1.49 & -4.51 & 13.23 & -8.98 & 6.78 & -0.46 & 11.83 \\
\hline lucene-2.0 & 54.66 & 2.07 & 4.89 & 10.28 & 12.69 & -7.06 & 3.3 & -0.46 & 5.65 \\
\hline forrest-0.8 & 58.6 & -45.08 & -58.6 & -0.69 & 12.55 & -53.25 & -36.66 & 11.68 & -3.7 \\
\hline poi-2.0 & 59.14 & 5.87 & -5.9 & 7.93 & 11.7 & 5.91 & -5.89 & 7.39 & 7.03 \\
\hline ant-1.3 & 64.94 & -0.73 & -12.64 & 4.9 & 11.21 & -6.74 & -27.9 & -5.32 & 3.31 \\
\hline ivy-1.1 & 63.18 & -0.01 & 0.15 & -15.3 & 11.03 & 4.92 & -4.58 & -17.42 & 7.42 \\
\hline ant-1.6 & 64.24 & 3.69 & 3.18 & -12.68 & 10.48 & 1.91 & -5.43 & -0.55 & 9.27 \\
\hline ant-1.5 & 63.68 & 2.14 & 5.67 & -9.85 & 9.19 & 2.2 & 7.97 & -16.96 & 9.04 \\
\hline xalan-2.4 & 65.74 & 2.11 & 4.61 & -1.34 & 8.8 & 1.01 & -3.24 & -0.55 & 6.52 \\
\hline xalan-2.7 & 42.05 & -9.23 & -3.89 & -2.48 & 6.18 & -4.64 & -3.57 & -10.38 & 4.91 \\
\hline ivy-1.4 & 72.38 & -2.6 & 2.42 & -5.85 & 5.71 & 1.59 & -2.52 & 0.6 & 3.37 \\
\hline ant-1.7 & 73.43 & -0.13 & 1.16 & -0.93 & 4.69 & -4.36 & -8.46 & -4.67 & -0.31 \\
\hline ant-1.4 & 58.93 & -5.59 & -7.83 & -2.37 & 1.76 & -5.54 & -16.63 & -9.81 & 4.68 \\
\hline poi-1.5 & 59.72 & -5.19 & -10.94 & -8.48 & 4.54 & -18.99 & -13.8 & -14.99 & -2.58 \\
\hline forrest- 0.7 & 56.55 & -23.86 & -23.64 & -38.32 & 4.2 & -32.16 & -39.48 & -19.81 & 3.72 \\
\hline pbeans-2.0 & 60.74 & 0.8 & -0.31 & -1.86 & 3.88 & -21.42 & -42.9 & -13.2 & -3.66 \\
\hline xalan-2.6 & 55.15 & -8.71 & -2.28 & -5.45 & 2.79 & -7.48 & -6.98 & -6.69 & 0.97 \\
\hline xalan-2.5 & 46.76 & -4.64 & -1.22 & -3.23 & 2.71 & -11.75 & -2.23 & -8.63 & 2.27 \\
\hline ivy-2.0 & 76.73 & -1.62 & -5.97 & 0.66 & 0.46 & -5.75 & -17.21 & -2.27 & -1.41 \\
\hline Mean & 45.09 & -2.39 & -2.25 & -1.27 & $15.77^{\oplus}$ & -3.4 & -4.52 & 4.63 & $15.65^{\oplus}$ \\
\hline
\end{tabular}

for the methods noFilter, original Burak, original Peters, Burak FS and Peters FS (with reference to the Best FS column). The original methods perform slightly better than 
noFilter. The highest performances are presented by the filtering methods associated with FS. Considering $g \geq 60$, Peters FS and Burak FS presented successful performance for more than $50 \%$ of projects. For the threshold $g \geq 75$, the method Burak FS presented practical use for almost $20 \%$ of the projects.

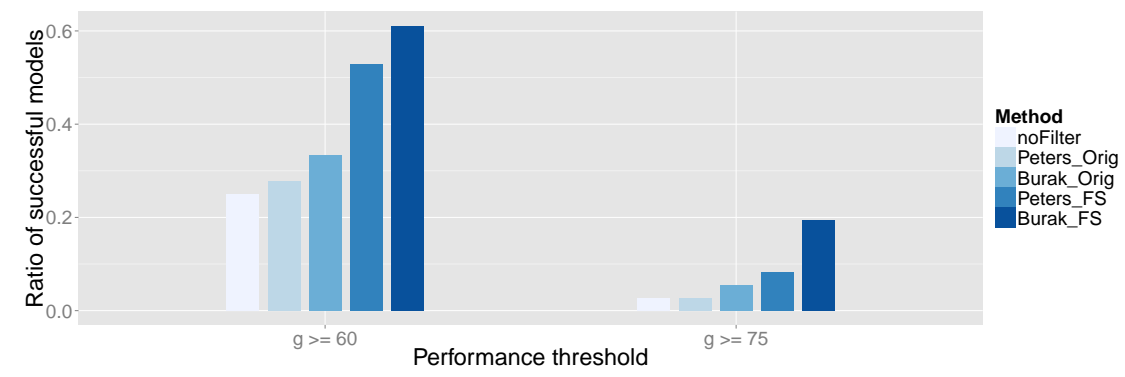

Figure 11 - Ratio of successful models for practical use considering the thresholds $g \geq 60$ and $g \geq 75$.

\section{Ranking}

Table 9 shows the performance obtained for each IFFS method considering the percentage of actual defects present in the top instances of the ranking. The respective cutoffs $\alpha$ and $\beta$ for each project can be viewed in Table 3. Table 9 shares the same visual structure as shown in Table 8 . The results are relative to noFilter, where $\Delta \alpha=\alpha-\alpha_{\text {noFilter }}$.

The column Random can be used as a comparative reference. In theory, as discussed in Yang, Tang and Yao (2015), supposing a context where the test resources are limited and allow to test only $20 \%$ of the software, the testers will spend it in the first $20 \%$ instances. In a random resource allocation, it is expected to cover only $20 \%$ of the total amount of defects in the first $20 \%$ instances. However, by prioritizing the most defective software parts it is possible to increase the percentage of covered defects. By comparing the random allocation shown in Table 9 with the $\beta$ cutoff shown in Table 3 , we can see coincident values (including the mean values of $25 \%$ ) which corroborates the expected theory. In addition, we can observe a strong learning of the noFilter models in relation to the Random models in which the performances are higher for all projects and the mean value is twice higher.

The column Best FS presented higher performance than noFilter with statistical significance - for both filters Burak and Peters. The original methods, however, presented lower performance than noFilter also with statistical significance. The Peters filter presented a general performance slightly better than the Burak filter if we consider the mean value of Best FS.

It is important to note that here we ignored all versions of the projects Forrest and Pbeans since their $\beta$ cutoff (see Table 3 ) shown to be very small for this kind of analysis. This sensitivity to the cutoff is one of the drawbacks of this performance measure. 
Table 9 - Performance comparison of the $\alpha$ percentage of defects in the top $\beta$ instances of Ranking, obtained for each evaluated method. The performances (except Random) are presented in relation to noFilter. Positive values mean better performances and negative values mean lower performances. The bold values indicate the highest performance for a project row. The symbols ${ }^{\oplus}$ and ${ }^{\ominus}$ represent the columns with significant difference in relation to noFilter.

\begin{tabular}{|c|c|c|c|c|c|c|c|c|c|c|}
\hline \multirow[b]{2}{*}{ Project } & \multicolumn{2}{|r|}{$(\alpha)$} & \multicolumn{4}{|c|}{ Burak Filter $(\Delta \alpha)$} & \multicolumn{4}{|c|}{ Peters Filter $(\Delta \alpha)$} \\
\hline & Random & noFilter & Orig & NET & CODE & Best FS & Orig & NET & CODE & Best FS \\
\hline ivy-1.4 & 5.37 & 32.59 & -0.56 & -2.59 & 6.67 & 12.59 & -4.81 & -2.96 & -1.85 & 13.52 \\
\hline synapse- 1.2 & 22.05 & 38.3 & 2.51 & 2.07 & 8.14 & 12.57 & 6.85 & -3.93 & 8.78 & 10.94 \\
\hline velocity-1.6 & 17.82 & 42.98 & 1.88 & 0.82 & -0.21 & 9.33 & -1.84 & 0.21 & 2.68 & 10.57 \\
\hline ant-1.4 & 16.3 & 26.67 & 0.07 & 3.84 & 8.48 & 10.29 & -4.49 & -1.45 & -3.12 & 8.55 \\
\hline synapse-1.1 & 18.42 & 43.1 & 0.88 & 4.11 & 2.49 & 7.74 & 6.57 & 6.06 & -2.15 & 10.27 \\
\hline ant- 1.7 & 16.06 & 53.74 & 4.1 & 3.81 & 0.8 & 5.68 & -5.23 & 1.4 & -2.54 & 8.08 \\
\hline lucene- 2.2 & 29.41 & 53.05 & -6.37 & -10.28 & -4.33 & 3.71 & -1.81 & -12.8 & 0.87 & 8 \\
\hline poi- 2.5 & 39.05 & 55.6 & -4.29 & 3.76 & 0.87 & 4.96 & 0.28 & -3.41 & -7.87 & 6.23 \\
\hline velocity-1.4 & 51.56 & 59.56 & -1.58 & -2.16 & -10.26 & 3.64 & 2.98 & 0.78 & -9.91 & 6.16 \\
\hline ant-1.5 & 10.67 & 36.67 & -6.48 & -0.48 & -0.86 & 5.9 & -7.14 & 1.9 & -13.81 & 2.57 \\
\hline ivy- 2.0 & 7.98 & 47.86 & -1.55 & -0.18 & -1.31 & 4.82 & 4.82 & 1.73 & -3.57 & 5.54 \\
\hline lucene-2.4 & 30.53 & 55.15 & -0.4 & 3.05 & 0.5 & 5.18 & 2.33 & 2.46 & 3.38 & 4.65 \\
\hline xerces-1.2 & 14.61 & 22.43 & -0.2 & -2.81 & 3.42 & 4.09 & 2.14 & -0.61 & 3.19 & 5.1 \\
\hline $\log 4 \mathrm{j}-1.0$ & 25.91 & 63.04 & -6.08 & -12.75 & 2.98 & 4.5 & -9.3 & -12.46 & -0.53 & 2.51 \\
\hline velocity-1.5 & 38.33 & 61.57 & -4.88 & -2.05 & -5.17 & 4.16 & -1.08 & -1.25 & -4.74 & 4.1 \\
\hline ant-1.6 & 15.45 & 52.46 & 1.59 & -0.78 & 1.36 & 4.13 & -0.24 & -0.67 & -4.13 & 2.14 \\
\hline poi-2.0 & 10.09 & 30.26 & 2.02 & -2.28 & -2.89 & 3.77 & 1.05 & -1.58 & 2.19 & 2.19 \\
\hline xalan-2.4 & 12.86 & 40.7 & -2.13 & -2.24 & -4.51 & 1.62 & -0.2 & -4.85 & -2.18 & 3.67 \\
\hline xerces-1.4 & 33.63 & 66.04 & -7.56 & -3.06 & -3.96 & 1.77 & -7.52 & -3.11 & -1.15 & 3.66 \\
\hline ant-1.3 & 12.22 & 38.08 & -1.92 & -0.2 & -6.77 & 3.54 & 3.23 & -2.63 & -4.95 & -0.3 \\
\hline xerces-1.3 & 11.97 & 46.55 & 0.1 & 1.14 & 1.62 & 1.62 & 0.78 & -0.48 & -0.31 & 3.25 \\
\hline synapse-1.0 & 8.1 & 40.63 & -15.56 & -11.59 & -13.33 & -0.63 & -14.92 & -7.46 & 3.02 & 3.17 \\
\hline xalan- 2.6 & 28 & 51.74 & -2.17 & -0.74 & -3.14 & 2.66 & -0.56 & -1.75 & -1.3 & 3.05 \\
\hline lucene-2.0 & 22.79 & 59.7 & -7.3 & -8.98 & -3.18 & 0.82 & -5.63 & -12.77 & -2.54 & 2.01 \\
\hline xalan- 2.5 & 35.78 & 52.2 & -1.1 & -0.65 & -1.72 & 1.85 & -2.58 & -2.46 & 1.04 & 1.04 \\
\hline poi-3.0 & 40.03 & 64.91 & 1.09 & -2.54 & -0.38 & 1.59 & -5.89 & -4.68 & -1.82 & 1.11 \\
\hline ivy-1.1 & 26.09 & 72.09 & -1.97 & -0.77 & 0.37 & 0.99 & -2.76 & -4.96 & -8.5 & -1.46 \\
\hline $\log 4 \mathrm{j}-1.2$ & 65.26 & 74.46 & -1 & -1.68 & 0.03 & 0.81 & -4.38 & -8.06 & -1.74 & -0.89 \\
\hline xalan- 2.7 & 74.01 & 79.58 & -0.39 & -0.03 & 0.02 & 0.13 & -0.3 & -1.15 & -0.05 & -0.02 \\
\hline $\log 4 \mathrm{j}-1.1$ & 23.21 & 70.42 & -22.19 & -14.51 & -5.15 & -3.12 & -2.57 & -2.11 & 0.08 & 0.08 \\
\hline poi-1.5 & 29.36 & 52.96 & -0.6 & -1.67 & -1.32 & -0.43 & -8.7 & -6.36 & -1.02 & -0.4 \\
\hline Mean & 25.58 & 51.13 & -2.65 & -2.01 & -0.99 & $3.88^{\oplus}$ & $-1.97^{\ominus}$ & $-2.88^{\ominus}$ & -1.76 & $4.16^{\oplus}$ \\
\hline
\end{tabular}

Furthermore, the ordering beyond the $\beta$ cutoff is ignored by this measure. In order to provide a more accurate analysis we also considered the FPA performance measure.

Table 10 shows the relative performances obtained for each evaluated method. The columns Max and Random are comparative references. Max is the reference for the actual number of defects and perfect ranking with the highest possible FPA. Again, the column Best FS presents better performances for both filters in relation to noFilter, with statistical significance. The results presented in Table 10 show similar patterns of performances that corroborate for a positive answer for RQ3.1. 
Table 10 - Performance comparison based on the ranking measure FPA. The columns Max and Random are comparative references. All other performances are presented in relation to noFilter. Positive values mean better performances and negative values mean lower performances. The symbols ${ }^{\oplus}$ and ${ }^{\ominus}$ represent the columns with significant difference in relation to noFilter.

\begin{tabular}{|c|c|c|c|c|c|c|c|c|c|c|c|}
\hline \multirow[b]{2}{*}{ Project } & \multicolumn{3}{|c|}{ (FPA) } & \multicolumn{4}{|c|}{ Burak Filter $(\triangle$ FPA $)$} & \multicolumn{4}{|c|}{ Peters Filter $(\triangle \mathrm{FPA})$} \\
\hline & $\operatorname{Max}$ & Random & noFilter & Orig & NET & CODE & Best FS & Orig & NET & CODE & Best FS \\
\hline forrest- 0.6 & 100 & 56.11 & 16.67 & 16.11 & 16.67 & 0 & 62.22 & 83.33 & 10.56 & 70.56 & 83.33 \\
\hline forrest- 0.7 & 96.78 & 46.98 & 69.23 & 9.73 & 2.35 & 13.51 & 22.96 & 12.86 & 13.54 & 8.28 & 18.68 \\
\hline forrest- 0.8 & 98.96 & 51.01 & 71.42 & -11.63 & -19.79 & 6.25 & 19.58 & 13.37 & 5.87 & 20 & 20 \\
\hline xerces-1.2 & 92.46 & 49.82 & 49 & -1.01 & 0.73 & 0.73 & 6.38 & 5.68 & 3.35 & 2.57 & 10.95 \\
\hline poi- 2.0 & 94.29 & 50.43 & 65.77 & 1.65 & -4.02 & 0.72 & 1.47 & -0.97 & -8.91 & -7.82 & 6.55 \\
\hline synapse-1.2 & 88.19 & 50.27 & 62.73 & -0.22 & -1.36 & 2.84 & 6.3 & 1.66 & -4.31 & 4.28 & 4.28 \\
\hline xerces-1.3 & 95.37 & 49.64 & 73.97 & 1.83 & 2.3 & 2.11 & 5.63 & 4.63 & 5.45 & 2.79 & 6.13 \\
\hline velocity-1.4 & 72.37 & 50.38 & 53.93 & -0.62 & -1.72 & -6.68 & 1.78 & 1.84 & 0.56 & -5.64 & 4.42 \\
\hline xerces-1.4 & 84.01 & 50.25 & 70.66 & -1.54 & -1.56 & 0.49 & 1.96 & -4.69 & 0.84 & 1.23 & 3.9 \\
\hline poi-2.5 & 77.21 & 49.82 & 62.37 & -1.63 & 1.95 & -1.11 & 1.96 & 0.34 & -1.2 & -3.15 & 3.42 \\
\hline synapse-1.0 & 96.22 & 50.46 & 76.73 & -4.96 & -2.52 & -1.65 & 3.15 & -9.92 & -2.41 & 1.02 & 3.07 \\
\hline ant-1.4 & 90.76 & 49.9 & 61.34 & -1.45 & 0.93 & 1.92 & 3.08 & -1.87 & -0.54 & -3.07 & 2.73 \\
\hline $\log 4 \mathrm{j}-1.0$ & 88.66 & 50.1 & 75.68 & -4.21 & -10.77 & 2.95 & 2.95 & -0.91 & -7.02 & 2.19 & 2.19 \\
\hline velocity- 1.5 & 79.67 & 49.65 & 65.65 & -2.22 & -0.61 & -2.66 & 2.07 & 0.13 & 1.8 & -0.48 & 2.82 \\
\hline lucene-2.4 & 83.11 & 50.64 & 68.13 & -0.32 & 1.03 & 0.33 & 1.54 & 1.08 & -1.05 & 2.65 & 2.65 \\
\hline ant-1.7 & 91.11 & 49.48 & 79.05 & 0.86 & 0.73 & -0.92 & 1.16 & -2.81 & 0.62 & -1.8 & 2.64 \\
\hline ant-1.5 & 95.1 & 48.64 & 77.43 & -1.93 & 0.72 & -2.14 & 2.41 & -4.52 & -0.04 & -3.03 & 1.22 \\
\hline lucene- 2.2 & 84.77 & 52.13 & 67.98 & -3.47 & -5.01 & -1.82 & 1.15 & -1.46 & -6.85 & -0.88 & 2.35 \\
\hline poi-1.5 & 84.01 & 51.91 & 64.85 & -1.61 & -0.08 & 0.27 & 2.06 & -4.3 & -3.36 & 0.63 & 0.67 \\
\hline synapse-1.1 & 90.72 & 49.65 & 71.96 & -0.18 & -0.41 & 0.36 & 1.95 & -1.66 & -2.92 & -4.04 & 2.04 \\
\hline pbeans- 1.0 & 74.89 & 51.94 & 69.79 & -1.58 & -3.4 & -6.44 & 0.84 & -5.54 & -3 & -6.14 & 2.03 \\
\hline ant-1.6 & 91.35 & 49.68 & 79.32 & 1.3 & 1.11 & -0.03 & 1.87 & -0.27 & 0.02 & -3.13 & 1.19 \\
\hline ivy-1.4 & 97.21 & 49.33 & 86.29 & -0.2 & 0.84 & -0.3 & 1.4 & -6.59 & -1.19 & -3.12 & 1.54 \\
\hline $\log 4 \mathrm{j}-1.1$ & 87.64 & 49.48 & 79.22 & -5.28 & -2.68 & 0.31 & 0.53 & 1.27 & 1.06 & 1.31 & 1.31 \\
\hline xalan-2.6 & 84.8 & 49.59 & 66.73 & -0.71 & -0.11 & -0.2 & 1.28 & -0.04 & -0.12 & -0.35 & 0.74 \\
\hline $\log 4 \mathrm{j}-1.2$ & 64.41 & 50.85 & 56.2 & -0.63 & -1.11 & 0.49 & 1.05 & -0.18 & -5.01 & 1.27 & 1.27 \\
\hline velocity-1.6 & 89.78 & 50.13 & 71.02 & -2.05 & -1.26 & -4.32 & 1.1 & -3.55 & -3.75 & -3.96 & 1.25 \\
\hline ant-1.3 & 94.38 & 49.8 & 77.88 & -8.09 & -2.23 & -2.73 & 1.16 & 1.05 & -5.2 & -2.93 & -1.43 \\
\hline pbeans- 2.0 & 94.22 & 49.72 & 78.15 & -0.7 & -2.99 & -6.36 & 0.9 & -7.16 & -4.32 & -0.35 & 0.31 \\
\hline xalan- 2.4 & 92.94 & 51.38 & 76.23 & -1.37 & -1.1 & -2.29 & 0.75 & -1.42 & -2.64 & -1.54 & -0.29 \\
\hline ivy-2.0 & 95.67 & 50.79 & 83.88 & -0.67 & -0.75 & -0.1 & 0.63 & -1.04 & -0.61 & -1.93 & -0.4 \\
\hline poi-3.0 & 80.64 & 50.2 & 68.63 & 0.21 & -0.49 & -0.78 & 0.45 & -3.01 & -2.29 & -2.44 & 0.27 \\
\hline xalan-2.5 & 80.94 & 50.15 & 61.65 & -0.24 & -0.36 & -0.35 & 0.42 & -1.93 & -2.37 & -0.63 & 0.41 \\
\hline lucene-2.0 & 88.13 & 50.47 & 74.56 & -1.49 & -1.7 & -0.34 & 0.09 & -1.97 & -5.1 & -0.79 & -0.02 \\
\hline xalan-2.7 & 59.36 & 50.45 & 56.1 & -0.64 & -0.1 & 0 & 0.08 & -0.37 & -0.76 & 0 & 0 \\
\hline ivy-1.1 & 86.69 & 49.37 & 80.17 & -0.73 & -0.25 & -0.8 & 0.05 & -1.03 & -2.16 & -3.34 & -1.08 \\
\hline Mean & 87.41 & 50.29 & 68.62 & $-0.82^{\ominus}$ & -1.03 & $-0.24^{\ominus}$ & $4.57^{\oplus}$ & 1.67 & -0.93 & $1.62^{\oplus}$ & $\mathbf{5 . 3 1} 1^{\oplus}$ \\
\hline
\end{tabular}

\section{RQ3.2: Which IFFS methods present better performances on CPDP?}

For both contexts (Classification and Ranking), the best performances obtained for each project are not dominated by one or just a few IFFS methods. Instead, each project has exclusive characteristics that promote one or other IFFS method. Thus, we analyzed the best performances by counting the frequency in which a method is among the top five best performances for a project. We consider all the 41 analyzed methods, 
$((17 F S+2 S S+$ Orig $) * 2$ filters $)+$ noFilter. By this approach we can show the results more clearly. The methods with higher frequency leads to better performances for a larger amount of software projects.

Table 11 - Frequency in which a IFFS method performs among the top five prediction performances for a project (Classification).

\begin{tabular}{lclllc}
\hline Method & Freq. & Method & Freq. & Method & Freq. \\
\hline BURAK_FS_IG_10 & 11 & PETERS_FS_RLF_5 & 6 & BURAK_FS_IG_20 & 4 \\
\hline PETERS_SS_CODE & 10 & PETERS_FS_IG_5 & 6 & BURAK_SS_NET & 4 \\
\hline BURAK_FS_IG_5 & 9 & PETERS_FS_RLF_15 & 6 & PETERS_FS_SPC_Te_5 & 4 \\
\hline PETERS_FS_SPC_Tr_5 & 9 & PETERS_FS_IG_10 & 5 & BURAK_FS_RLF_15 & 4 \\
\hline BURAK_FS_SPC_Te_5 & 8 & NO_FILTER & 5 & PETERS_FS_RLF_10 & 4 \\
\hline BURAK_FS_RLF_10 & 7 & PETERS_FS_IG_15 & 5 & PETERS_FS_IG_20 & 3 \\
\hline BURAK_FS_SPC_Tr_10 & 7 & BURAK_FS_SPC_Te_20 & 5 & BURAK_FS_RLF_5 & 3 \\
\hline BURAK_FS_SPC_Te_10 & 7 & BURAK_FS_SPC_Te_15 & 5 & PETERS_SS_NET & 3 \\
\hline BURAK_SS_CODE & 6 & PETERS_FS_SPC_Te_10 & 5 & BURAK_FS_CFS & 3 \\
\hline BURAK_FS_SPC_Tr_5 & 6 & BURAK_FS_SPC_Tr_15 & 4 & PETERS_FS_SPC_Te_20 & 3 \\
\hline
\end{tabular}

Table 12 - Frequency in which a IFFS method performs among the top five prediction performances for a project (Ranking).

\begin{tabular}{lclllc}
\hline Method & Freq. & Method & Freq. & Method & Freq. \\
\hline BURAK_FS_SPC_Tr_10 & 10 & BURAK_FS_RLF_5 & 5 & BURAK_FS_IG_20 & 4 \\
\hline BURAK_FS_SPC_Tr_5 & 9 & PETERS_FS_IG_10 & 5 & BURAK_ORIG & 4 \\
\hline BURAK_FS_IG_10 & 9 & BURAK_FS_IG_15 & 5 & PETERS_FS_IG_20 & 4 \\
\hline PETERS_SS_CODE & 9 & PETERS_FS_SPC_Te_5 & 5 & BURAK_FS_SPC_Te_15 & 4 \\
\hline NO_FILTER & 8 & PETERS_FS_SPC_Tr_10 & 5 & PETERS_SS_NET & 4 \\
\hline BURAK_SS_CODE & 8 & PETERS_FS_CFS & 5 & PETERS_FS_SPC_Tr_20 & 4 \\
\hline BURAK_FS_SPC_Te_10 & 8 & BURAK_FS_SPC_Te_5 & 4 & PETERS_FS_SPC_Te_20 & 4 \\
\hline PETERS_FS_IG_15 & 6 & BURAK_FS_CFS & 4 & BURAK_FS_SPC_Te_20 & 3 \\
\hline PETERS_FS_SPC_Tr_15 & 6 & PETERS_FS_IG_5 & 4 & PETERS_FS_RLF_10 & 3 \\
\hline PETERS_ORIG & 5 & BURAK_FS_IG_5 & 4 & BURAK_SS_NET & 3 \\
\hline
\end{tabular}

Tables 11 and 12 show the 30 methods with highest frequencies among the best performances for Classification and Ranking, respectively. The Ranking frequencies are based on the FPA measure since it covers the performance of all projects and also has shown to be more consistent in this context.

In the Classification context, the method with the highest frequency (BURAK_FS_IG_10, where the postfix 10 represents the $k$ configuration used on FS_IG) covers almost $30 \%$ of all projects, followed by PETERS_SS_CODE with frequency 10. These two methods also appear between the 10 most frequent methods for Ranking. Indeed, we can observe some similarities by comparing the two contexts. For example, 6 of the 10 most frequent methods for Classification are also between the 10 most frequent methods for Ranking. The original filter methods (with no feature subset selection) presented frequencies lower than noFilter in both prediction contexts. Actually, the performance of noFilter appears between the 15 and 5 highest frequencies for Classification and Ranking, 
respectively. This result partially contradicts the results presented by Turhan et al. (2009) and Peters, Menzies and Marcus (2013), in which the instance filtering improves the prediction in relation to noFilter. However, some differences can be highlighted between their works and ours. First, we assumed that both original methods consider all features on filtering instances. However, in their works the experiments are conducted considering only the CODE metrics set since only this set is available (i.e., the NET metrics set is not available for analysis). Here, we can observe that SS_CODE presented good performances since it appears between the 10 most frequent methods for both contexts and filters.

In addition, the experiments of Turhan et al. (2009) are on the cross-company context and the analyzed projects were developed by different companies. The projects considered on this study were generally developed by the Apache Foundation (except for the Pbeans project). This aspect can influence on the level of heterogeneity of data and then improve the performance of prediction by using noFilter. Lastly, Peters, Menzies and Marcus (2013) restrict the testing domain to software projects with less than 100 instances, differently of us (see Tables 4 and 3).

By observing the filters separately, we can note that the Burak filter is present in 5 of the first 7 most frequent methods in both contexts. Those frequencies, however, are not mutually exclusive and may count the same project for several methods. For example, in the classification context it is necessary 10 of the first most frequent methods to cover all the 36 projects.

\subsection{Threats to Validity}

The results indicate that the performance of CPDP models can be improved by applying the evaluated methods. However, some sources of bias can be highlighted from the conducted experiments. First, the evaluated collection of datasets is composed only by Java open source projects. Also, the majority of the evaluated projects belongs to a unique company. These bias can reduce the heterogeneity of data and influence the experiment results. Other datasets with different sources can be used in order to improve the generality of results.

Another important threat to validity is that the number of defects found for each software part is an approximate estimation and does not represent the real number of existing defects (JURECZKO; MADEYSKI, 2010; MADEYSKI; JURECZKO, 2014). However, a precise information in this context is difficult, if not impossible, to acquire in a real project (YANG; TANG; YAO, 2015). Kitchenham, Mendes and Travassos (2007) argue that software companies frequently do not keep suitable historical information about software quality. When available, those information are commonly private or restricted only for internal use (ZIMMERMANN; NAGAPPAN, 2008). 
Despite these factors, some positive points justify the use of this collection of data: 1) the datasets are open for reuse; 2) the independent variables can be automatically extracted; 3) the collection is composed of several project versions, allowing us to conduct the experiments in the CPDP context; 4) the data acquisition is based on a systematic and frequently used process (ZIMMERMANN; PREMRAJ; ZELLER, 2007; ZHANG et al., 2014; MADEYSKI; JURECZKO, 2014); and 5) these data were extensively reused in the literature (JURECZKO; MADEYSKI, 2010; HE et al., 2013; PRATEEK; PASALA; ARACENA, 2013; HERBOLD, 2013; MADEYSKI; JURECZKO, 2014; ZHANG et al., 2016).

Also, the models in this study were constructed by using only one machine learning algorithm, sustained by the performance results presented in Lessmann et al. (2008), Jiang, Cukic and Ma (2008), Weyuker, Ostrand and Bell (2010), and Yang, Tang and Yao (2015). However, the data mining is an active research field and other algorithms may lead to different results.

\subsection{Conclusion}

In this chapter we investigated the performance of CPDP models by applying filtering methods associated with feature subset selection in both prediction contexts Classification and Ranking. We evaluated 17 methods derived from different configurations of four distinct Feature Selection methods. The evidence obtained from the results are similar in both prediction contexts. The results show that for all the analyzed projects at least one of the 17 evaluated methods presented better performance in relation to both the original filtering methods and the absent of filtering methods.

In the Classification context, the percentage of models considered successful for practical use was improved. In the Ranking context, besides to present better performances in relation to the comparative reference, it was possible to observe the evident learning of defect patterns when compared with random ordering.

We also investigated which of the evaluated methods present better performance. We show a list of the evaluated methods ordered by the frequency in which a method is between the best top five performances for a project. The most frequent methods are present in only 11 (Classification) and 10 (Ranking) of the 36 analyzed projects. It indicates that the most appropriate method can vary for each project.

The study presented in this chapter accredits the investigation of which characteristics better represent a software and how these information can be used in order to predict the most appropriate IFFS method for a project.

In the next chapter we expand this study to the general context of CPDP. We 
evaluate the performance of 31 CPDP methods, including the Burak filter and other five CPDP solutions associated with five different classifiers (including the Random Forest). We expand the collection of data to 47 versions of 15 Java open source projects. A new performance measure and a new statistical test are considered. 

CHAPTER

4

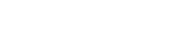

\section{PERFORMANCE EVALUATION OF CPDP \\ METHODS}

\subsection{Introduction}

Several solutions were proposed in the literature aiming at improving the predictive performance of CPDP models. From these solutions, we highlight the transfer learning solutions (PAN; YANG, 2010). This approach is characterized by the use of some knowledge about the target domain in order to approximate the different distributions of source and target data, as discussed in Section 2.4. Different strategies are explored in this context, such as the transformation of data (WATANABE; KAIYA; KAIJIRI, 2008; CRUZ; OCHIMIZU, 2009), the filtering of relevant instances or projects (TURHAN et al., 2009; HERBOLD, 2013; HE et al., 2013), or the different weighting of relevant training instances (MA et al., 2012).

To the best of our knowledge, no comprehensive comparison among these transfer learning solutions was conducted in the literature. Existing comparative analyzes are restricted to a few methods (MA et al., 2012; HE et al., 2013; HERBOLD; TRAUTSCH; GRABOWSKI, 2016) or do not provide a uniform experimental setup (GUNARATHNA; TURHAN; HOSSEINI, 2016). In the systematic literature review presented in (GUNARATHNA; TURHAN; HOSSEINI, 2016), the authors compared the performance of CPDP methods based on the information provided by each published work. However, the considered studies diverge in terms of data, classifiers, and performance measures. The absence of a uniform experimental setup precludes concrete comparisons of performances between studies on a large scale. In addition, most of these methods can be associated to different machine learning algorithms, composing variations with different predictive performances. The knowledge about which methods are typically best can assist practitioners on choosing a method to use. 
In this chapter, we provide an extensive experimental comparison of 31 CPDP methods applied to 47 versions of 15 open source software projects. The 31 methods derived from the combination of six state-of-the-art transfer learning solutions with the five most frequently used classifiers in the defect prediction literature. We designed a uniform experimental setup to mitigate potential sources of bias that could accidentally lead to conclusion instability (MENZIES; SHEPPERD, 2012; SONG; MINKU; YAO, 2013; SHEPPERD; BOWES; HALL, 2014; TANTITHAMTHAVORN et al., 2016).

The contribution of this experimental analysis is twofold: 1) it provides the first comprehensive evaluation of performance for the state-of-the-art CPDP methods, covering different classification algorithms and transfer learning methods; and 2) the results show that, despite the fact that a group of methods generally present better predictive performance than other methods across datasets, the most suitable method can vary according to the project being predicted - accrediting the meta-learning solution proposed in Chapter 5 .

We aim at answering the following research questions:

RQ4.1 Which CPDP methods perform better across datasets?

RQ4.2 Do the best CPDP methods perform better for the same datasets?

The experiments and data analysis were implemented and conducted with $\mathrm{R}^{1}$.

This chapter is organized as follows. In Section 4.2, we discuss the experimental setup and issues involved in this experimentation. In Section 4.3, we present and discuss the obtained results. In Section 4.4, we discuss the threats to the validity of the conducted experiment. In Section 4.5 we summarize the obtained conclusions.

\subsection{Experimental Setup}

\subsubsection{Software Projects}

The conducted experiments are based on 47 versions of 15 Java open source projects, provided by Madeyski and Jureczko (2014). This collection of datasets is an updated version of the collection presented in Section 3.4.1. The datasets and detailed information about the software projects are available in the $\operatorname{link}^{2}$ provided by the authors. Each instance in a dataset represents a Java Object-Oriented class (OO class). We converted the dependent variable to a binary classification problem $(1$, for number of defects $>0$; or 0, otherwise) (HE et al., 2013). Table 13 lists the number of instances, number of defects, and defect rate for each of the analyzed datasets.

$1<$ https://www.r-project.org/>

2 < http://purl.org/MarianJureczko/MetricsRepo/> 
Table 13 - Summary of the projects characteristics.

\begin{tabular}{|c|c|c|c|}
\hline Project & \# Instances & \# Defective Instances & \% Defective Instances \\
\hline ant-1.3 & 180 & 20 & 0.11 \\
\hline ant-1.4 & 253 & 39 & 0.15 \\
\hline ant-1.5 & 381 & 32 & 0.08 \\
\hline ant-1.6 & 496 & 92 & 0.19 \\
\hline ant-1.7 & 999 & 166 & 0.17 \\
\hline camel-1.0 & 409 & 13 & 0.03 \\
\hline camel-1.2 & 687 & 209 & 0.3 \\
\hline camel-1.4 & 1009 & 144 & 0.14 \\
\hline camel-1.6 & 1117 & 184 & 0.16 \\
\hline ckjm-1.8 & 10 & 5 & 0.5 \\
\hline forrest- 0.7 & 31 & 5 & 0.16 \\
\hline forrest-0.8 & 34 & 2 & 0.06 \\
\hline ivy-1.1 & 131 & 63 & 0.48 \\
\hline ivy-1.4 & 311 & 16 & 0.05 \\
\hline ivy-2.0 & 459 & 40 & 0.09 \\
\hline jedit-3.2.1 & 492 & 90 & 0.18 \\
\hline jedit-4.0 & 579 & 75 & 0.13 \\
\hline jedit-4.1 & 616 & 79 & 0.13 \\
\hline jedit-4.2 & 764 & 48 & 0.06 \\
\hline jedit-4.3 & 1049 & 8 & 0.01 \\
\hline $\log 4 j-1.0$ & 152 & 34 & 0.22 \\
\hline $\log 4 \mathrm{j}-1.1$ & 122 & 37 & 0.3 \\
\hline $\log 4 j-1.2$ & 258 & 187 & 0.72 \\
\hline lucene-2.0 & 276 & 91 & 0.33 \\
\hline lucene-2.2 & 355 & 141 & 0.4 \\
\hline lucene-2.4 & 497 & 199 & 0.4 \\
\hline pbeans-1.0 & 32 & 20 & 0.62 \\
\hline pbeans- 2.0 & 63 & 10 & 0.16 \\
\hline poi-1.5 & 237 & 130 & 0.55 \\
\hline poi-2.0RC1 & 304 & 36 & 0.12 \\
\hline poi-2.5.1 & 383 & 223 & 0.58 \\
\hline poi-3.0 & 441 & 257 & 0.58 \\
\hline synapse-1.0 & 158 & 16 & 0.1 \\
\hline synapse-1.1 & 223 & 60 & 0.27 \\
\hline synapse-1.2 & 257 & 86 & 0.33 \\
\hline tomcat-6.0.389418 & 1052 & 77 & 0.07 \\
\hline velocity-1.4 & 205 & 132 & 0.64 \\
\hline velocity-1.5 & 225 & 133 & 0.59 \\
\hline velocity-1.6.1 & 237 & 76 & 0.32 \\
\hline xalan-2.4.0 & 818 & 110 & 0.13 \\
\hline xalan-2.5.0 & 873 & 365 & 0.42 \\
\hline xalan-2.6.0 & 869 & 326 & 0.38 \\
\hline xalan-2.7.0 & 888 & 732 & 0.82 \\
\hline xerces-1.2.0 & 426 & 64 & 0.15 \\
\hline xerces-1.3.0 & 451 & 69 & 0.15 \\
\hline xerces-1.4.4 & 573 & 378 & 0.66 \\
\hline xerces-init & 193 & 67 & 0.35 \\
\hline Total & 20575 & 5386 & 0.26 \\
\hline
\end{tabular}

The set of independent variables is composed of the 20 code metrics, already presented in Table 5. This set of metrics has been reported as good quality indicators on the literature (MADEYSKI; JURECZKO, 2014). All the metrics are numerical and can be automatically extracted directly from the source code with the $\mathrm{Ckjm}^{3}$ tool. Further details can be found in Jureczko and Madeyski (2010) and Madeyski and Jureczko (2014).

$\overline{3<\text { http://gromit.iiar.pwr.wroc.pl/p_inf/ckjm }>~}$ 


\subsubsection{Data Preprocessing}

Data transformation is a resource used to re-express data in a new scale aiming at spreading skewed curves equally among the batches (CRUZ; OCHIMIZU, 2009). The result is a distribution near to the normal distribution assumption with less variability, better symmetry, reduced number of outliers and better effectiveness for data mining (CRUZ; OCHIMIZU, 2009; TURHAN et al., 2009). We applied a log transformation (CRUZ; OCHIMIZU, 2009; TURHAN et al., 2009). Each numeric value is replaced by its logarithm. Since some metrics present zero values which would result on infinitive values, we applied a simple solution as follows: $x^{\prime}=\log (x+1)$ (CRUZ; OCHIMIZU, 2009).

Log transformation provides a uniform data preprocessing for all projects since it is not dependent on the current test and training sets. In Nam, Pan and Kim (2013) and Keung, Kocaguneli and Menzies (2013), the authors discuss the considerable impact on performance caused by different preprocessing resources.

\subsubsection{CPDP Methods}

For this experiment we selected six state-of-the-art transfer learning solutions based on the benchmark proposed in Herbold (2013). We re-implemented each solution in R following their respective original publication instructions. We refer to each solution with the abbreviation [year][first author], as described below.

2008 Watanabe (WATANABE; KAIYA; KAIJIRI, 2008): the authors proposed a simple compensation method based on the mean value. Let $x_{i j}$ be the $j$ th feature of the test instance $x_{i}$, and mean $\left(\operatorname{Tr}_{j}\right)$ and mean $\left(T e_{j}\right)$ be the mean values of the $j$ th feature of the training and test sets, respectively. The compensated test metric value $x_{i j}^{\prime}$ is given by:

$$
x_{i j}^{\prime}=x_{i j} *\left[\operatorname{mean}\left(\operatorname{Tr}_{j}\right) / \operatorname{mean}\left(T e_{j}\right)\right] .
$$

The base-learner is then applied over the transformed data.

2009Cruz (CRUZ; OCHIMIZU, 2009): the authors proposed a similar compensation method based on the median value, associated with a log transformation (already defined in Section 4.2.2). Considering the notations defined above, the compensated test metric value $x_{i j}^{\prime}$ is given by:

$$
x_{i j}^{\prime}=x_{i j}+\left[\operatorname{median}\left(\operatorname{Tr}_{j}\right)-\operatorname{median}\left(\operatorname{Te}_{j}\right)\right] .
$$

2009Turhan (TURHAN et al., 2009): also known as KNN filter or Burak filter, this solution applies the k-nearest neighbor algorithm to select the most similar instances based on the Euclidean distance. For each test instance, the solution searches for the $k$ most similar training instances (from all available projects). The selected instances 
compose the filtered training set. Duplicated instances are discarded. As in the original proposal, we set $k=10$.

2013He (HE et al., 2013): this solution is composed of three steps. First, the $N$ projects datasets most similar to the test set are selected to compose the training data. Let $D_{\text {train }}^{i}$ be the dataset of project $i(1<i<N)$ and $D_{\text {test }}$ the test set. In this step, a new dataset called $S A M_{i}$ with $2 K$ instances is constructed containing $K$ instances from $D_{\text {train }}^{i}$ and $K$ instances from $D_{\text {test }}$. The dependent variable of $S A M_{i}$ is a binary label differentiating the training instances from the test instances. The similarity between $D_{\text {train }}^{i}$ and $D_{\text {test }}$ is calculated based on the accuracy (rate of correct predictions) in which a classifier is able to differentiate (or separate) between training and test instances in $S A M_{i}$. In this step we applied a Logistic Regression as the separability classifier and set $N=10$, as suggested by the authors.

Second, for each of the project datasets selected in the previous step, the ratio of $F S S * 100$ percent of unstable features are removed, where $F S S$ is a predefined parameter. The same dataset $S A M_{i}$ is used to measure the information gain of each feature in relation to the dependent variable. The features with higher information gains are considered more unstable and are removed. It is important to note that each of the selected projects (step 1) generates a new training set with possibly a distinct subset of features ( step 2). We set $F S S=0.8$, as suggested by the authors. Finally, the authors apply an ensemble strategy with majority voting to combine the predictions given by the selected training sets (obtained from steps 1 and 2). For each selected training set, a model is constructed using a classifier. Then, for each test instance, a prediction score is generated for each constructed model. In the original proposal, a threshold of 0.5 is applied for each prediction score and the majority voting defines the final binary prediction.

In our implementation, since we are working with the AUC measure (obtained from the prediction score), we considered the average prediction score obtained from the ensemble strategy instead of the majority voting.

2013Herbold (HERBOLD, 2013): in the original work, the authors proposed and evaluated two distinct solutions. We chose to implement and evaluate in this study only the solution reported with better performance. Similar to the KNN filter, this solution is also based on the k-nearest neighbor algorithm. However, instead of filtering individual instances, this solution filters the most similar projects. Each project is represented by its distributional characteristics, given by the mean and standard deviation of each feature. The authors suggest a neighborhood size of $50 \%$ from the available projects to compose the training data.

2012Ma (MA et al., 2012): also known as Transfer Naïve Bayes, this solution introduces the concept of data gravitation to prioritize and weight training instances. 
The weights of the training instances are inversely proportional to their distances from the test set. Given the indicator $h$ :

$$
h\left(a_{i j}\right)= \begin{cases}1, & \text { if } \min _{j} \leq a_{i j} \leq \max _{j} \\ 0, & \text { otherwise }\end{cases}
$$

where $a_{i j}$ is the $j$ th feature of the training instance $x_{i}$, and $\min _{j} / \max _{j}$ are the minimum/maximum values of the $j$ th feature across all test instances. The function $h$ indicates whether $a_{i j}$ is within the range of values of the test instances. The distance $s_{i}$ of a training instance from the test set is given by:

$$
s_{i}=\sum_{j=1}^{k} h\left(a_{i j}\right)
$$

where $k$ is the number of features. Then, the weighting measure is given by:

$$
w_{i}=\frac{s_{i}}{\left(k-s_{i}+1\right)^{2}}
$$

According to this formula, higher weights are given to the more similar training instances. A weighted Naïve Bayes classifier is then constructed according to the weights given by $w_{i}$.

The solutions 2008Watanabe, 2009Turhan, 2009Cruz, 2013He and 2013Herbold are not dependent on a specific classifier. The solution 2012Ma, however, is intrinsically associated to the Naïve Bayes algorithm. Therefore, in order to provide a fair comparison, we combined each of the independent solutions with the five most popular classifiers in the literature of defect prediction, according to Malhotra (2015): Random Forest (RF); Support Vector Machines (SVM); Multilayer Perceptron (MLP); C4.5 (C45); and Naïve Bayes (NB). We briefly describe each of these classifiers in Section 2.3.

For all five classifiers we used the implementation and original parameters provided in the RWeka Package ${ }^{4}$. As a baseline, we also evaluate the performance of each classifier in its original form (Orig), with no transfer learning solution. In total, we evaluate 31 methods, as illustrated in Figure 12.

\subsubsection{Performance Measure}

The normal procedure for the binary classification of instances is determined in two steps. First, the classifier returns a probability of an instance be positive for a specific class (e.g, defective). Then, a cutoff (or prediction threshold) is applied to determine the final prediction. In the SDP context, probabilities greater than the cutoff value are considered defective. Traditional measures, such as the pd (recall) and pf (probability of false alarm) 


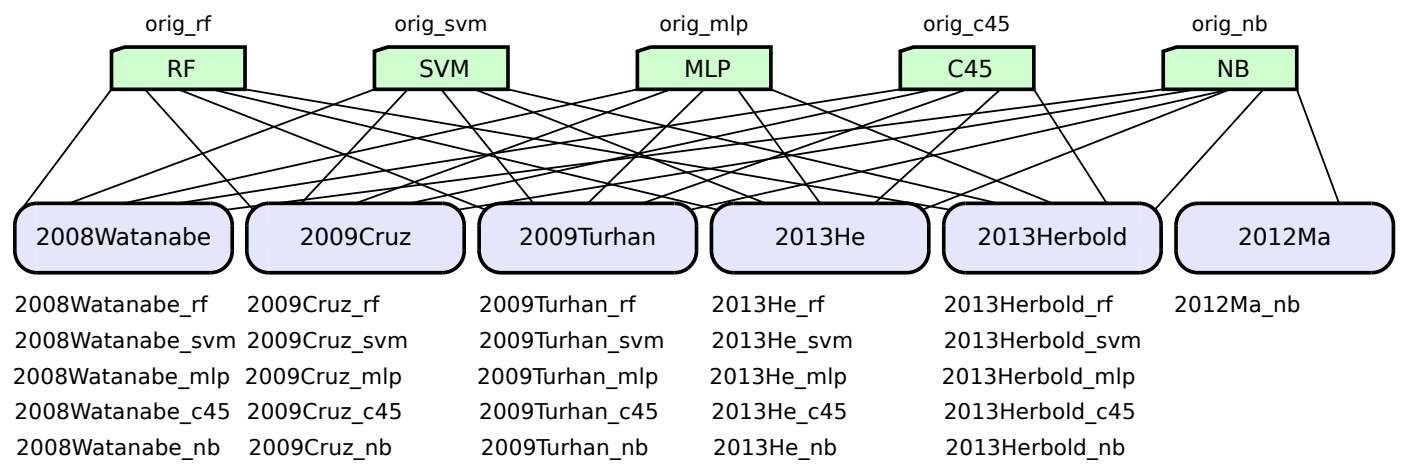

Figure 12 - All 31 evaluated CPDP methods derived from the combination of six transfer learning solutions and five classifiers.

defined in Section 3.4.5, are highly influenced by the defined threshold. Commonly, this parameter is configured to 0.5 .

The experiments conducted in this chapter are based on the AUC measure. This measure is derived from the Receiver Operating Characteristic (ROC) curve. The ROC curve is plotted over the pd and pf values generated by varying the prediction threshold (see Figure 13). The AUC measure is the area under the ROC curve. An AUC value of 1 means a perfect classifier and a 0.5 means a random classifier.

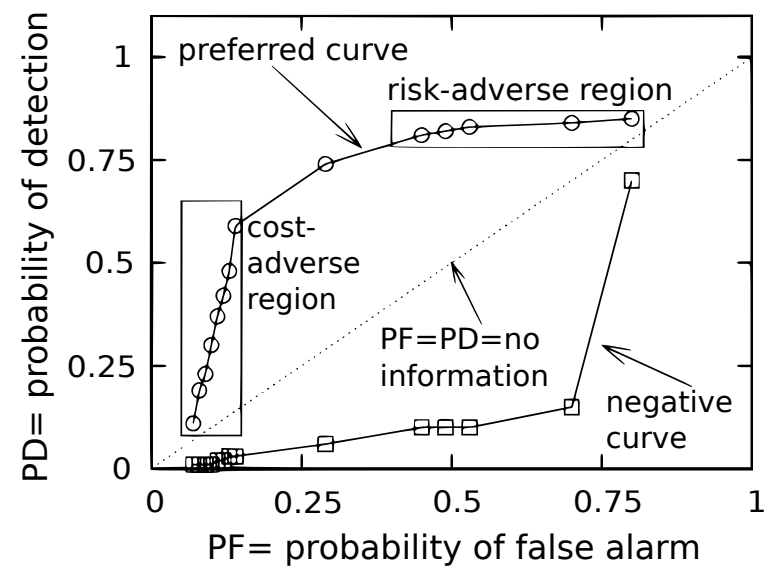

Figure 13 - Regions of a typical ROC curve (MENZIES; GREENWALD; FRANK, 2007).

We choose this performance measure for three main reasons. First, the AUC measure provides a single scalar value balancing the influence of true positive rate (also called recall or probability of detection) and false positive rate (also called probability of false alarm) (MA et al., 2012). The use of a single value facilitates the comparison across models. Second, the AUC measure is not sensitive to the thresholds used as cutoff parameters, which can introduce bias on the performance analysis (GUNARATHNA; TURHAN; HOSSEINI, 2016). Lastly, the AUC measure is also robust to class imbalance, which is frequently present in software defect prediction (MALHOTRA, 2015). The datasets used in this study also present class imbalance, as shown in Table 13. 


\subsubsection{Experiment Design}

The experiment design is presented in Figure 14. We aim to evaluate the 31 CPDP methods applied to all the 47 project versions. For this, we follow the leave-one-out cross-validation variation called CPLOO, presented in Section 3.4.3. In this procedure, the training set contains all the available project versions except the versions of the same project of the current test set.

The use of this procedure is important considering our experimentation context: to predict defects in a project based on defect patterns from external projects. This approach is also named strict CPDP in the literature (HERBOLD; TRAUTSCH; GRABOWSKI, 2016).

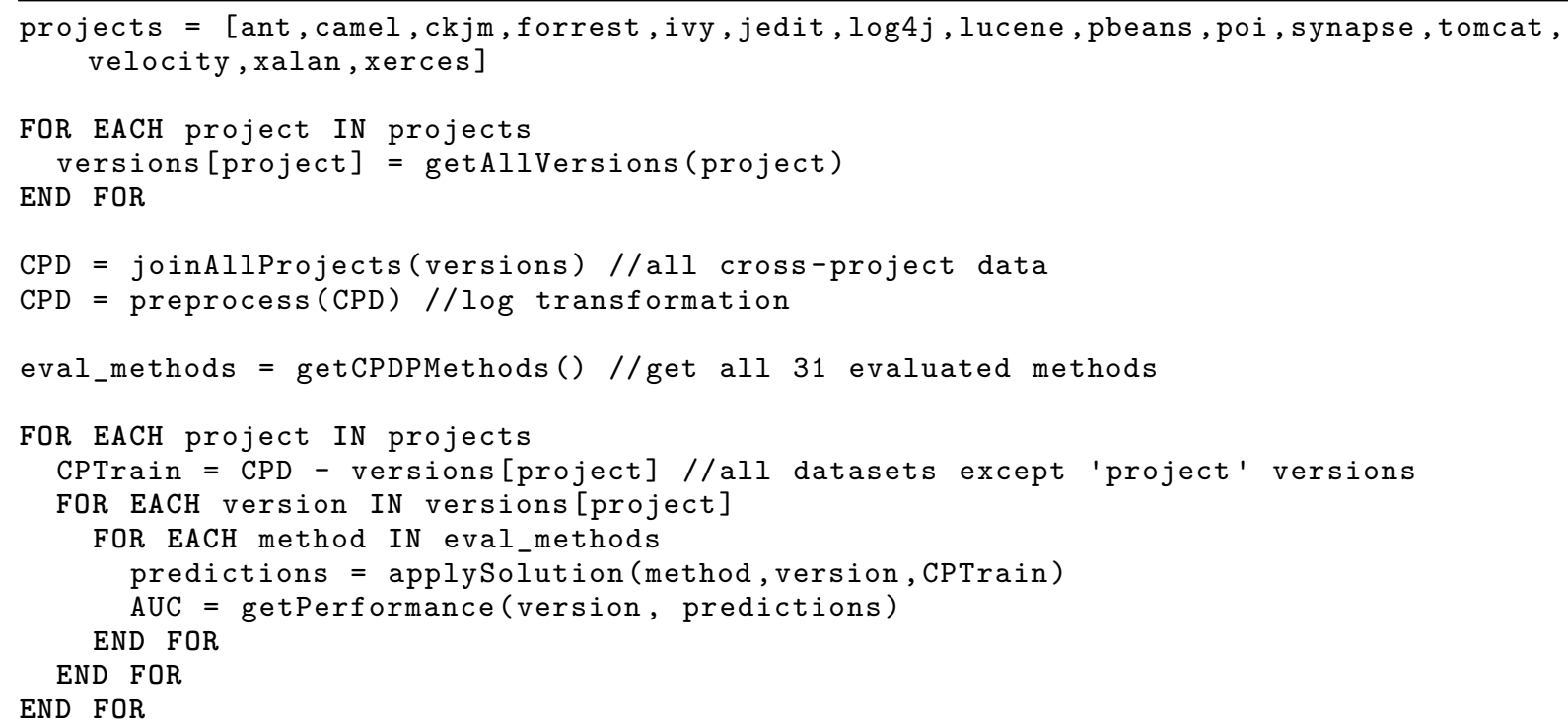

Figure 14 - Pseudocode of the experiment design with Cross-Project Leave One Out (CPLOO) cross-validation.

\subsubsection{Statistical Test}

For the statistical analysis we applied Friedman test followed by Fisher's LSD test (PEREIRA; AFONSO; MEDEIROS, 2015). The use of Friedman test followed by a Post-hoc analysis is recommended for the comparison of multiple solutions over multiple datasets (DEMSAR, 2006).

The performances are organized as a table of rankings in which the rows represent the datasets (project versions), the columns represent the compared CPDP methods, and each cell is filled with the respective performance ranking position of a method for a dataset. In this configuration, tied performances share the average ranking position. For example, the following AUC performances $[0.79,0.79,0.77,0.76,0.75,0.75]$ are ranked as $[1.5,1.5,3,4,5.5,5.5]$. 
As mentioned in Larson (2006), the number of significant digits is justified by the specific research purpose, the measurement accuracy and sample size. In this statistical test, we rounded the AUC performances to 2 significant digits in order to not differentiate very similar performances.

The Friedman test verifies whether the ranking performances of all methods are statistically equivalent. When the null hypotheses is refused, a Post-hoc analysis is applied to define the solutions with significant difference of performance. Fisher's LSD test is indicated as the most powerful method in this scenario according to a recent study (PEREIRA; AFONSO; MEDEIROS, 2015). In this test, we considered the confidence of $95 \%$ (i.e., $p$-value $\leq 0.05$ ).

\subsection{Results}

\section{RQ4.1: Which CPDP methods perform better across datasets?}

Two main factors are compared in this performance analysis. First, we extracted the AUC performance for each of the 31 CPDP methods applied for all the 47 software projects (or datasets). Then, we extracted the ranking by ordering the AUC performances of the evaluated methods for each dataset. Table 14 presents the performance mean (and standard deviation) for each CPDP method. The methods are ordered according to the mean rank.

First, we can observe a generally better performance (across datasets) of CPDP methods based on the NB learner, including orig_nb. All the seven methods combined with NB performed among the top 10 positions. In contrast, the performances based on the C45 classifier performed among the last positions, excepting for 2013He_c45 (position 17). The other classifiers are spread along mixed positions with no clear assignments. Although the first positions in this table present close AUC mean values, it is clear the distance between the best and worse AUC mean performances. The method on the last position performed close to a random classifier (when $\mathrm{AUC}=0.5$ ). In this study, we assume a successful model, for practical use, when AUC $\geq 0.75$. Although the success of a predictive model is relative to the application domain, it is commonly set to a performance greater than $75 \%$ in the CPDP literature (ZIMMERMANN et al., 2009; PETERS; MENZIES; MARCUS, 2013; PORTO; SIMAO, 2016a).

As discussed in Section 4.2.6, we analyzed the statistical significance of results based on the Friedman test. The null hypothesis assumes all performances as equivalent. The alternative hypothesis is that at least one pair of predictive models has different performances. The null hypothesis is rejected with a $p$-value $<2.2 \mathrm{e}-16$. Therefore, we analyze the pairwise difference of performances with the Fisher's LSD test. The result 
Table 14 - Performance obtained for each of the 31 CPDP methods considering the mean (and standard deviation) values for all 47 software projects.

\begin{tabular}{|c|c|c|c|}
\hline Pos. & Method & Mean Rank & Mean AUC \\
\hline 1 & 2012Ma_nb & $7.57( \pm 5.63)$ & $0.771( \pm 0.10)$ \\
\hline 2 & $2013 \mathrm{He} \_r f$ & $8.26( \pm 6.02)$ & $0.766( \pm 0.09)$ \\
\hline 3 & 2009Turhan_nb & $9.48( \pm 6.12)$ & $0.761( \pm 0.09)$ \\
\hline 4 & 2013He_svm & $9.90( \pm 6.15)$ & $0.765( \pm 0.09)$ \\
\hline 5 & 2013Herbold_nb & $10.00( \pm 5.86)$ & $0.760( \pm 0.09)$ \\
\hline 6 & 2009Cruz_nb & $10.38( \pm 6.03)$ & $0.757( \pm 0.09)$ \\
\hline 7 & orig_nb & $10.52( \pm 5.62)$ & $0.758( \pm 0.09)$ \\
\hline 8 & 2013He_nb & $11.47( \pm 5.88)$ & $0.756( \pm 0.09)$ \\
\hline 9 & 2008Watanabe_nb & $11.55( \pm 6.83)$ & $0.752( \pm 0.10)$ \\
\hline 10 & orig_rf & $11.74( \pm 6.00)$ & $0.756( \pm 0.09)$ \\
\hline 11 & 2008Watanabe_rf & $12.04( \pm 6.68)$ & $0.753( \pm 0.10)$ \\
\hline 12 & $2013 \mathrm{He} \_$_mlp & $12.82( \pm 8.10)$ & $0.753( \pm 0.09)$ \\
\hline 13 & 2013Herbold_rf & $12.84( \pm 7.10)$ & $0.748( \pm 0.08)$ \\
\hline 14 & 2009Turhan_rf & $13.03( \pm 7.56)$ & $0.745( \pm 0.10)$ \\
\hline 15 & orig_mlp & $14.86( \pm 7.37)$ & $0.739( \pm 0.09)$ \\
\hline 16 & 2009Cruz_rf & $14.97( \pm 5.92)$ & $0.737( \pm 0.10)$ \\
\hline 17 & $2013 \mathrm{He} \_\mathrm{c} 45$ & $15.73( \pm 8.27)$ & $0.734( \pm 0.09)$ \\
\hline 18 & 2013Herbold_mlp & $17.00( \pm 6.96)$ & $0.729( \pm 0.08)$ \\
\hline 19 & 2009Turhan_svm & $17.72( \pm 9.38)$ & $0.702( \pm 0.13)$ \\
\hline 20 & 2009Cruz_svm & $18.17( \pm 7.00)$ & $0.717( \pm 0.10)$ \\
\hline 21 & orig_svm & $18.17( \pm 7.00)$ & $0.717( \pm 0.10)$ \\
\hline 22 & 2008Watanabe_mlp & $18.78( \pm 6.99)$ & $0.714( \pm 0.09)$ \\
\hline 23 & 2008Watanabe_svm & $19.14( \pm 7.05)$ & $0.706( \pm 0.11)$ \\
\hline 24 & 2013Herbold_svm & $19.26( \pm 9.58)$ & $0.680( \pm 0.13)$ \\
\hline 25 & 2009Cruz_mlp & $21.10( \pm 6.92)$ & $0.693( \pm 0.10)$ \\
\hline 26 & 2009Turhan_mlp & $21.31( \pm 8.26)$ & $0.688( \pm 0.10)$ \\
\hline 27 & orig_c45 & $24.87( \pm 6.88)$ & $0.639( \pm 0.09)$ \\
\hline 28 & 2013Herbold_c45 & $25.06( \pm 6.99)$ & $0.636( \pm 0.10)$ \\
\hline 29 & 2009Turhan_c45 & $25.16( \pm 7.22)$ & $0.633( \pm 0.10)$ \\
\hline 30 & 2009Cruz_c45 & $25.69( \pm 6.82)$ & $0.624( \pm 0.09)$ \\
\hline 31 & 2008Watanabe_c45 & $27.39( \pm 4.77)$ & $0.602( \pm 0.08)$ \\
\hline
\end{tabular}

is presented in Figure 15. The alphabet letters group the methods with no significant difference.

No significant difference was found between 2012Ma_nb and the next 3 methods although it presents better performance in relation to all remaining methods - including orig_ $n b$. This differentiation in relation to the original learner is important since it elucidates the real gain produced by each transfer learning solution. For example, except for 2012Ma_nb, no significant difference between the CPDP methods using NB and orig_nb was found - as stated in group 'c'.

This relation between transfer learning solutions and their respective learner baseline is better exposed in Table 15. When there is no significant difference, the relation is represented by the symbol "/". Otherwise, it is filled with " $(+)$ " or " $(-)$ ", meaning a better or worse mean rank position, respectively.

From this table, we can observe that most of the applied transfer learning solutions do not lead to significant difference of performance in relation to their respective original classifiers. Moreover, some solutions diminish the performances when associated to a learner, as observed for the MLP classifier with the solutions 2008Watanabe, 2009Cruz, and 2009Turhan. On the other hand, the solution 2013He significantly improves the 


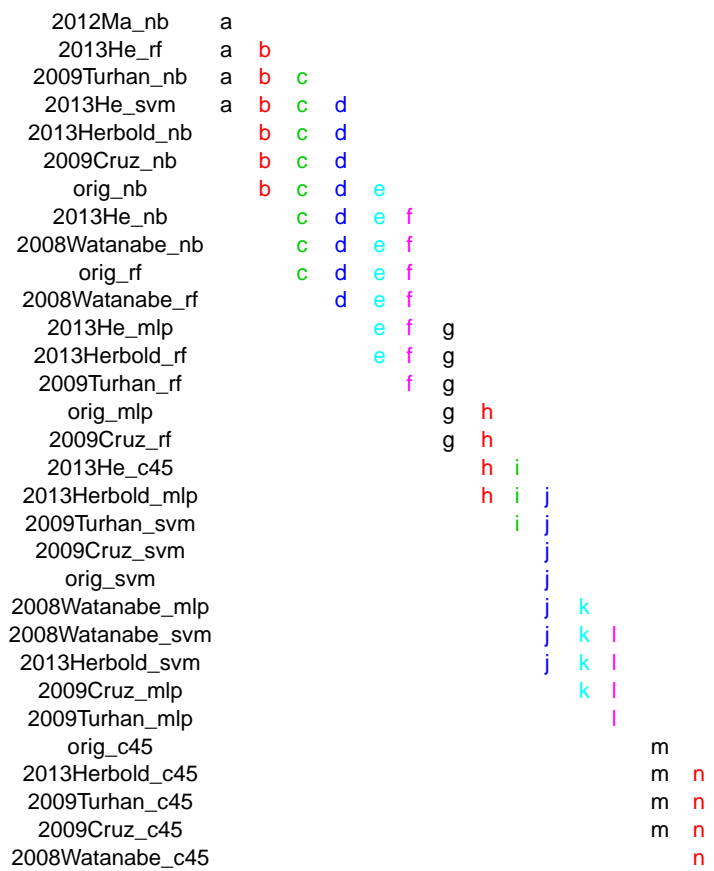

Figure 15 - Pairwise Post-hoc Fisher's LSD test. Each letter represents a group of methods with no statistical significant difference.

Table 15 - Comparison of CPDP methods in relation to the baseline original learners, based on the statistical Fisher's LSD test. The symbol "/" means no statistical difference. The symbols " $(+)$ " or "(-)" mean statistical difference with better or worse mean rank, respectively.

\begin{tabular}{lccccc}
\hline & rf & svm & mlp & $\mathbf{c 4 5}$ & nb \\
\hline orig & - & - & - & - & - \\
\hline 2008Watanabe & $/$ & $/$ & $(-)$ & $(-)$ & $/$ \\
\hline 2009Cruz & $(-)$ & $/$ & $(-)$ & $/$ & $/$ \\
\hline 2009Turhan & $/$ & $/$ & $(-)$ & $/$ & $/$ \\
\hline 2013He & $(+)$ & $(+)$ & $/$ & $(+)$ & $/$ \\
\hline 2013Herbold & $/$ & $/$ & $/$ & $/$ & $/$ \\
\hline 2012Ma & - & - & - & - & $(+)$ \\
\hline
\end{tabular}

performances of the classifiers RF, SVM, and C45. The solution 2012Ma also improves the performance when associated to the NB classifier.

From the statistical analysis presented in Figure 15, the methods 2012Ma_nb, 2013He_rf, 2009Turhan_nb, and 2013He_svm presented the best performances across datasets although the method 2009Turhan nb did not present significant difference in relation to its respective original learner baseline.

However, other criteria can be considered for a comparative analysis. For example, in their original published works (MA et al., 2012; HE et al., 2013), both 2012Ma and $2013 H e$ presented lower computation time cost in relation to the 2009Turhan solution, although $2013 \mathrm{He}$ presented higher complexity in relation to 2012Ma. On the other hand, $2013 \mathrm{He}$ is more robust to redundant and irrelevant features in relation to 2012Ma. First, because $2013 H e$ has an internal procedure to filter the most relevant features. In addition, both classifiers RF and SVM are known to be robust in this context. Second, because 
2012Ma is sensitive to redundant and irrelevant features in two points: its internal weighting procedure, based on the features relation between testing and training instances; and its assumption of independence between features inherited from the Naïve Bayes algorithm.

\section{RQ4.2: Do the best CPDP methods perform better for the same datasets?}

To answer this question, we make use of the information presented in Figure 15. We evaluate the performances of the CPDP methods: 2012Ma_nb, 2013He_rf, 2009Turhan_$n b$, and 2013He_svm; referring to the four methods with better ranking across datasets. Then, for each dataset, we associate the best AUC performance obtained among these four CPDP methods. This association of best methods for each dataset is presented in Table 16. As already mentioned in Section 4.2.6, the AUC performance comparison is based on 2 significant digits. In this way, we do not differentiate very similar performances.

We can extract some information from this table. First, the best CPDP method for a project version is not necessarily the same for all versions of the same project. Second, more than one CPDP method can achieve the best AUC performance for the same project version. Third, from all the 47 project versions: $72 \%$ achieved a successful performance with $\mathrm{AUC} \geq 0.75 ; 21 \%$ performed in the range $0.65 \leq \mathrm{AUC}<0.75$; and only $6 \%$ performed with AUC below 0.65. Last but not least, there is no general better performance. This statement is better visualized in Figure 16. In this figure, each CPDP method represents a set of all projects in which it achieved the best performance. The intersections between sets (i.e when a project version has more than one best method) are represented by the connected dots.

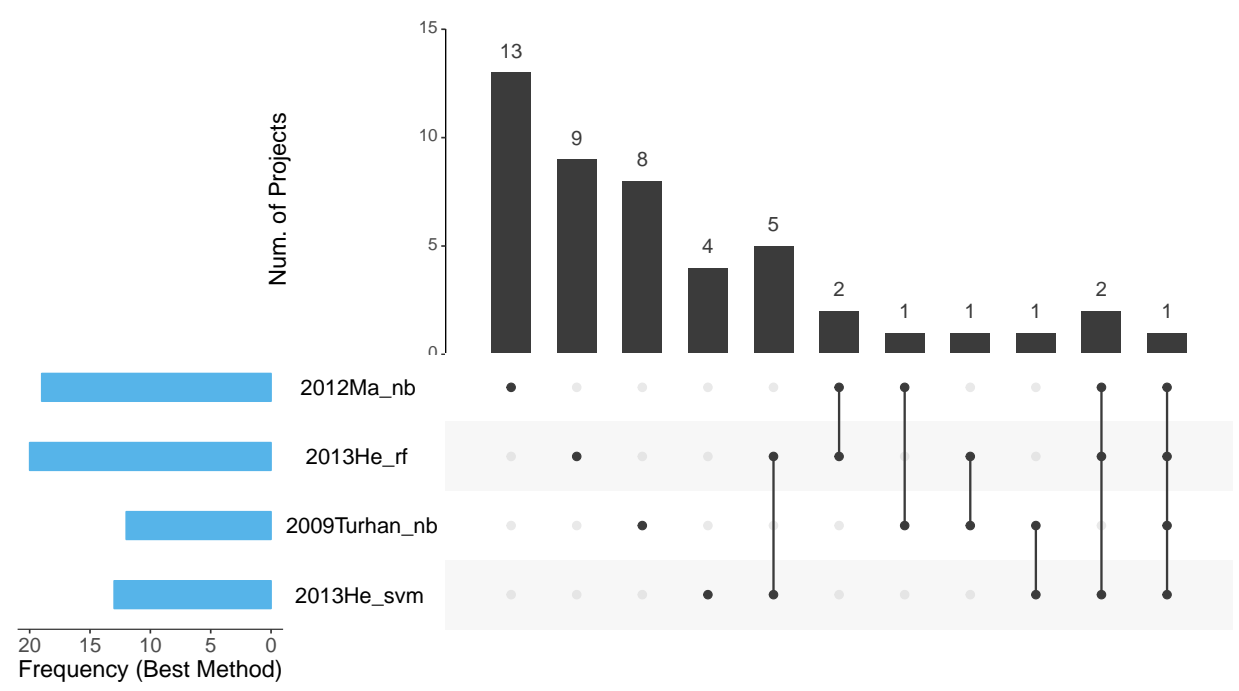

Figure 16 - Data of Table 16 organized by intersection sets. Each CPDP method represents a set of all projects in which it achieved the best performance. The bars represent the distribution of all 47 projects along the four methods. When a project has more than one best method, the intersection is represented by the connected dots. 
Table 16 - Best AUC value and respective best CPDP methods by project, considering the four methods with better ranking across datasets. The AUC values were rounded to 2 significant digits in order to not differentiate similar performances.

\begin{tabular}{|c|c|c|c|}
\hline Project & Best AUC & \# Best Methods & Best Methods \\
\hline ant-1.3.csv & 0.88 & 1 & 2012Ma_nb \\
\hline ant-1.4.csv & 0.68 & 1 & 2012Ma_nb \\
\hline ant-1.5.csv & 0.84 & 1 & $2012 \mathrm{Ma}$ nb \\
\hline ant-1.6.csv & 0.86 & 1 & $2012 \mathrm{Ma} \_\mathrm{nb}$ \\
\hline ant-1.7.csv & 0.85 & 1 & 2012Ma_nb \\
\hline camel-1.0.csv & 0.84 & 1 & 2009Turhan_nb \\
\hline camel-1.2.csv & 0.64 & 1 & $2013 \mathrm{He} \_r f$ \\
\hline camel-1.4.csv & 0.74 & 1 & 2009Turhan_nb \\
\hline camel-1.6.csv & 0.68 & 1 & $2013 \mathrm{He} \_r f$ \\
\hline ckjm-1.8.csv & 0.96 & 1 & $2013 \mathrm{He} \_$svm \\
\hline forrest-0.7.csv & 0.81 & 1 & 2009Turhan_nb \\
\hline forrest-0.8.csv & 0.91 & 1 & $2013 \mathrm{He} \_s v m$ \\
\hline ivy-1.1.csv & 0.81 & 2 & $2013 \mathrm{He} \_r f, 2012 \mathrm{Ma} \_n b$ \\
\hline ivy-1.4.csv & 0.8 & 1 & $2013 \mathrm{He} \_r f$ \\
\hline ivy-2.0.csv & 0.85 & 2 & 2012Ma_nb, 2013He_rf \\
\hline jedit-3.2.1.csv & 0.89 & 1 & 2012Ma_nb \\
\hline jedit-4.0.csv & 0.87 & 2 & 2013He_rf, 2009Turhan_nb \\
\hline jedit-4.1.csv & 0.91 & 2 & $2013 \mathrm{He} \_r f, 2013 \mathrm{He} \_$svm \\
\hline jedit-4.2.csv & 0.92 & 4 & 2013He_rf, 2012Ma_nb, 2009Turhan_nb, 2013He_svm \\
\hline jedit-4.3.csv & 0.9 & 1 & $2013 \mathrm{He} \_$svm \\
\hline $\log 4 \mathrm{j}-1.0 . \mathrm{csv}$ & 0.89 & 1 & 2009Turhan_nb \\
\hline $\log 4 \mathrm{j}-1.1 . \mathrm{csv}$ & 0.86 & 1 & 2012Ma_nb \\
\hline $\log 4 \mathrm{j}-1.2 . \mathrm{csv}$ & 0.88 & 1 & $2012 \mathrm{Ma}$ nb \\
\hline lucene-2.0.csv & 0.81 & 1 & $2012 \mathrm{Ma} \_\mathrm{nb}$ \\
\hline lucene-2.2.csv & 0.75 & 2 & $2013 \mathrm{He} \_$svm, 2013He_rf \\
\hline lucene-2.4.csv & 0.8 & 2 & $2013 \mathrm{He} \_r f, 2013 \mathrm{He} \_s v m$ \\
\hline pbeans-1.0.csv & 0.68 & 1 & 2009Turhan_nb \\
\hline pbeans-2.0.csv & 0.81 & 1 & 2009Turhan_nb \\
\hline poi-1.5.csv & 0.76 & 1 & $2012 \mathrm{Ma} \_n b$ \\
\hline poi-2.0RC1.csv & 0.7 & 2 & $2013 \mathrm{He} \_s v m, 2013 \mathrm{He} \_r f$ \\
\hline poi-2.5.1.csv & 0.79 & 1 & 2012Ma_nb \\
\hline poi-3.0.csv & 0.85 & 3 & $2013 \mathrm{He} \_r f, 2013 \mathrm{He} \_$svm, 2012Ma_nb \\
\hline synapse-1.0.csv & 0.79 & 1 & $2012 \mathrm{Ma} \_\mathrm{nb}$ \\
\hline synapse-1.1.csv & 0.68 & 3 & 2012Ma_nb, 2013He_svm, 2013He_rf \\
\hline synapse-1.2.csv & 0.77 & 1 & 2009Turhan_nb \\
\hline tomcat-6.0.389418.csv & 0.82 & 1 & $2012 \mathrm{Ma} \_n b$ \\
\hline velocity-1.4.csv & 0.62 & 2 & $2013 \mathrm{He} \_$svm, $2013 \mathrm{He} \_r f$ \\
\hline velocity-1.5.csv & 0.71 & 2 & 2012Ma_nb, 2009Turhan_nb \\
\hline velocity-1.6.1.csv & 0.76 & 1 & $2013 \mathrm{He} \_$svm \\
\hline xalan-2.4.0.csv & 0.81 & 1 & $2013 \mathrm{He} \_r f$ \\
\hline xalan-2.5.0.csv & 0.68 & 1 & $2013 \mathrm{He} \_r f$ \\
\hline xalan-2.6.0.csv & 0.76 & 1 & $2013 \mathrm{He} \_r f$ \\
\hline xalan-2.7.0.csv & 0.85 & 1 & $2013 \mathrm{He} \_r f$ \\
\hline xerces-1.2.0.csv & 0.53 & 1 & $2013 \mathrm{He} \_r f$ \\
\hline xerces-1.3.0.csv & 0.72 & 1 & 2009Turhan_nb \\
\hline xerces-1.4.4.csv & 0.76 & 2 & 2009Turhan_nb, 2013He_svm \\
\hline xerces-init.csv & 0.68 & 1 & $2013 \mathrm{He} \_r f$ \\
\hline Mean & 0.791 & 1.36 & - \\
\hline
\end{tabular}

From all the 47 project versions: $28 \%$ achieved the best performance exclusively with 2012Ma_nb; 19\% with 2013He_rf; 17\% with 2009Turhan_nb; 9\% with 2013He_svm; and $27 \%$ share the best performance with more than one CPDP method. As we can see, no method always performs best. 


\subsection{Threats to Validity}

Some factors can threat the validity of the experiments conducted in this chapter. The first issues are related to the collection of data used in the experiments. Any lack of quality in these data may jeopardize the entire study. These issues were already discussed in Section 3.6, which also apply to the experiments conducted in this chapter.

The methods evaluated in this study (including transfer learning solutions and classifiers) compose a representative sample of the state-of-the-art in CPDP. However, it does not represents an exhaustive comparison covering all the existing solutions. The extension of these experiments with additional methods can lead to alternative conclusions. Also, the internal parameters of each method follow either the default configuration provided by the code libraries or the original recommendations of the authors. The tuning of parameters can influence the performances and, consequently, the conclusions over results. Although the use of default configurations is a common approach in the experimental software engineering literature, future work should investigate the impact of parameter tuning on this analysis.

The preprocessing step also influences directly on the performance of predictive models (KEUNG; KOCAGUNELI; MENZIES, 2013). We set the same log transformation resource for all methods in order to diminish the impact of this step on results. However, some internal preprocessing resources can still interfere with performance. For example, the Naïve Bayes classifier operates in conjunction with data discretization; which may benefit this classifier over other (KEUNG; KOCAGUNELI; MENZIES, 2013). Also, Herbold (2013) argues that the performance of SVMs can be positively impacted by the weighting of imbalanced training data, which is ignored in this study.

\subsection{Conclusion}

In this chapter we conducted an experimental comparison of 31 CPDP methods derived from six state-of-the-art transfer learning solutions associated to the five most frequently used classifiers in the defect prediction literature. We presented a uniform experimental setup aiming at mitigating the likely conclusion instability. The experiment is based on 47 versions of 15 open source software projects. The performance is evaluated based on the AUC value. For the statistical analysis, we applied the Friedman test followed by the Fisher's LSD test.

From the obtained results, we managed to identify the four best ranked CPDP methods across datasets in terms of AUC: 2012Ma_nb, 2013He_rf, 2009Turhan_nb, and 2013He_svm. We also identified that these four methods presented best performances for distinct groups of datasets, evidencing that the most suitable method for a project depends 
on the project being predicted. These results accredit the investigation of new resources to assist the task of deciding which methods are most suitable for a given project.

In the next chapter we investigate to what extent a meta-learning solution could predict the best CPDP method according to the project characteristics. We propose a meta-learning architecture designed for the CPDP context and evaluate its performance. The proposed architecture and the conducted experiments are based on the results discussed in this chapter. 

CHAPTER

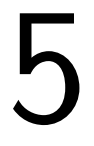

\section{META-LEARNING FOR CPDP}

\subsection{Introduction}

The experiments conducted in the previous chapters indicate that the most suitable method for a project can vary according to the project (or dataset) being predicted. This scenario is also encountered in the general machine learning research area. The no free lunch theorem states that no inductive bias is suitable for all domains (WOLPERT, 2002). This statement leads us to a new decision task concerning which is the most suitable solution for a given domain. This task is named algorithm recommendation (MANTOVANI et al., 2015; DORES et al., 2016; PARMEZAN; LEE; WU, 2017), which is part of the general meta-learning literature (LEMKE; BUDKA; GABRYS, 2015; BRAZDIL et al., 2017). A meta-learning model is characterized by its capacity of learning from previous experiences and adapt its bias dynamically according to the target domain (BRAZDIL et al., 2017). In the context of this study, a meta-learning model should be able to recommend a suitable CPDP method according to the characteristics of the software being predicted.

In this chapter, we propose and evaluate a different application of meta-learning, designed for the recommendation of CPDP methods. The process is based on the general meta-learning approach proposed in Kalousis (2002). Three main differences can be highlighted from previous studies: 1) the performances are obtained from external project datasets with CPLOO cross-validation instead of a cross-validation within the datasets (see Section 4.2.5); 2) we adopted unsupervised meta-features instead of the traditional supervised meta-features, commonly used in the literature (DORES et al., 2016) (see Section 5.2.2); and 3) the meta-target characterizes a multi-label classification task (TSOUMAKAS; KATAKIS, 2007) (see Section 5.2.3).

We consider two distinct meta-features sets for the characterization of datasets. Both sets are evaluated in two different levels. In the meta-level, we evaluate the learning 
capacity of each meta-features set in relation to a baseline. In the base-level, we evaluate the general performance of the proposed solution in relation to the four best CPDP methods applied individually.

We aim at answering the following research questions:

RQ5.1 To what extent can meta-learning help us to select the most suitable CPDP method for a given dataset?

RQ5.1.1 Does the meta-learner learn?

RQ5.1.2 How does the meta-learner perform across datasets?

This chapter is organized as follows. In Section 5.2, we present the general methodology and details related to the construction of the meta-model. In Section 5.3 we present the experiment design. In Section 5.4, we analyze and discuss the obtained results. In Section 5.5 we present the threats to validity. In Section 5.6, we summarize the obtained conclusions.

\subsection{Proposed Methodology}

Figure 17 presents the general architecture of the proposed solution. The meta-target is determined by the performances obtained from the input datasets. The meta-features are also extracted from the characterization of these datasets. Next to the meta-data preprocessing, a multi-label meta-learner is applied. This meta-learner is associated to a wrapper feature selection method in order to select a subset of meta-features to compose the final meta-model. The meta-model can then be used to recommend suitable CPDP methods for new project datasets. Once the meta-model is constructed, only the new project datasets need to be characterized and preprocessed before predicting a suitable CPDP method. More details and related issues are discussed in the following subsections.

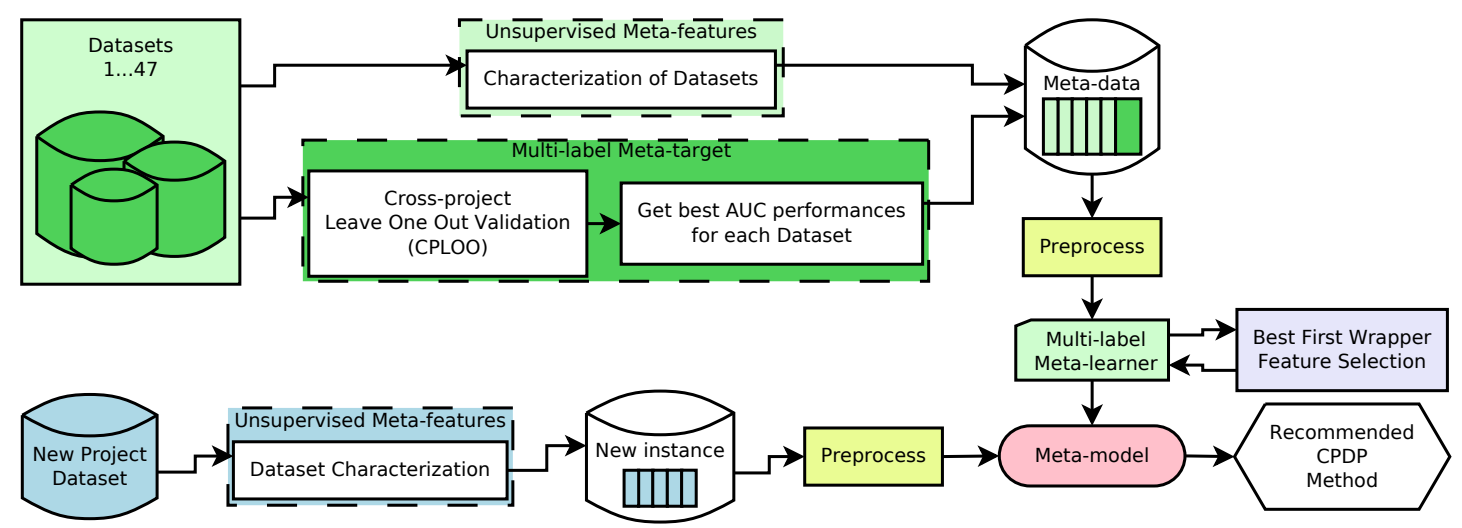

Figure 17 - Proposed architecture: meta-learning for CPDP. 


\subsubsection{Input Datasets}

The collection of datasets (meta-instances) is composed of the 47 projects already presented in Section 4.2.1. The amount of meta-instances is important since the more available information the more effective and generalizable can be the meta-model. However, as stated in Brazdil et al. (2017), scarce training data for the meta-learning task is a common aspect in this context. Although there is no recommended minimum amount of data, more than 50 datasets are desirable for a meta-learning analysis (DORES et al., 2016). Thereby, the available amount of data is a limitation for this study.

\subsubsection{Meta-features}

One of the main challenges for the construction of an efficient meta-learning model is the proper characterization of meta-instances (or datasets) (BRAZDIL et al., 2009). In this context, traditionally, each meta-instance represents a dataset referring to the training set (or source data) of a learning task. For example, consider the meta-model SFP-MLF proposed by Dores et al. (2016) for the recommendation of classification algorithms in the WPDP context. In their work, each meta-instance represents a different WPDP training set. Thus, given an unseen software project to be predicted (the target data), the characterization of its respective training set (or source data) is used as input for the SFP-MLF, which returns a suitable classifier as output. This approach, based on the source data, is appropriate in the WPDP context due to the assumption that the source and target data have the same distribution (see Section 2.4).

In the many-to-one CPDP context investigated in this work, this assumption is not valid. As discussed in Section 2.4, the training set (or source data) is composed of several different projects leading to a heterogeneous set. In addition, this same training set can be used to predict different software projects (or target data). Consider a CPDP context where the different unseen software projects are predicted based on the same heterogeneous training set. In this context, the characterization of datasets to compose the meta-data can not be based only on the training set since the training set does not provide the necessary information to map the relationship between the different unseen software projects and the performances of the different applied CPDP methods. In other words, the information about which CPDP method performs better for a project can not be extracted from the source data but from the target data.

Therefore, different from previous related work, the characterization of datasets in the context of this study is based on the target data information, i.e., each meta-instance of the meta-data represents a different test set. Thus, given an unseen software project to be predicted (target data), the characterization of the target data itself is used as input for the proposed meta-model, which returns a suitable CPDP method as output. 
A direct implication of this approach, based on the target data, is that the target data does not dispose of the target attribute information. Therefore, the traditional characterization of datasets based on supervised meta-features is not feasible in this context (see Section 2.6). Then, we propose the characterization of datasets based on unsupervised meta-features (non-dependent on the target attribute). However, the unsupervised characterization of datasets is little explored in the meta-learning literature (SANTOS; CARVALHO, 2016).

For this study, we evaluate two different sets of meta-features. Both are related to continuous data and can be automatically extracted from the original dataset.

The first meta-features set (here called MS-Dist) corresponds to the direct application of distributional measures over all the dataset features. For this, we consider 5 distributional measures: mean (mean), standard deviation (sd), median (med), maximum (max) and minimum (min). This approach leads to 100 meta-features, considering all 5 distributional measures applied for all the 20 features of a dataset. We also included the size (number of instances) of a dataset, totalizing 101 meta-features. The use of distributional measures to characterize datasets was already proposed in the CPDP literature but for different purposes (HE et al., 2012; HE et al., 2013; HERBOLD, 2013).

The second meta-features set (here called MS-Uns) is proposed in Santos and Carvalho (2016) and covers different characteristics of a dataset including general measures, statistical measures and clustering based measures. Originally, this set includes 53 unsupervised meta-features applied in the context of active learning. From the original set, we selected 44 measures applicable to the context of this study. The selected subset of meta-features is presented in Table 17 . The measures matching the pattern $M_{\Omega}$ (e.g., $\operatorname{sd}_{\text {max }}, \operatorname{corr}_{\min / \text { max }}$, skew mean) are obtained in two steps. First, the measure $M$ is extracted for each element of the dataset, generating a vector of values (e.g., the standard deviation extracted for all the features or the correlation extracted for all the pairs of features). Then, a distributional measure $\Omega$ (e.g., min, max) is applied over this vector of values, generating a single value. The numbers 1, 1.5, and 2 attached to the measures 'kurt', 'conn', 'dunn', and 'silh' represent an internal parametrization indicating the proportion of clusters per class.

\subsubsection{Meta-target}

The meta-target is obtained from the experiment results presented in Chapter 4 . From the 31 evaluated CPDP methods, we consider four possible labels for a project: 2012Ma_nb, 2013He_rf, 2009Turhan_nb, and 2013He_svm. These four methods refer to the best-ranked methods across datasets, as discussed in Section 4.3.

Another important characteristic in this study relates to the meta-target designed 
Table 17 - Unsupervised meta-features set (MS-Uns), where $\mathscr{U}$ represents a dataset with $n$ instances, $x \in \mathscr{U}$ represents an instance of $\mathscr{U}$, and $1<j<20$ represents a feature of $\mathscr{U}$ (Adapted from (SANTOS; CARVALHO, 2016)).

\begin{tabular}{|c|c|c|}
\hline Meta-feature & Name & Formula \\
\hline size & Size (number of instances) & $n=|\mathscr{U}|$ \\
\hline $\mathrm{size}_{l g}$ & Logarithm of size & $\log n$ \\
\hline $\operatorname{mean}_{\text {min }}$, mean $_{\text {max }}$, mean $_{\text {mean }}$, mean $_{\min / \max }$ & Mean & $\mu_{j}=\frac{1}{n} \sum_{x \in \mathscr{U}} x_{j}$ \\
\hline $\mathrm{sd}_{\min }, \mathrm{sd}_{\max }, \mathrm{sd}_{\text {mean }}, \mathrm{sd}_{\min / \max }$ & Standard deviation & $\sigma_{j}=\frac{1}{n} \sum_{x \in \mathscr{U}}\left(x_{j}-\mu_{j}\right)^{2}$ \\
\hline entr $_{\text {min }}$, entr $_{\text {max }}, \operatorname{entr}_{\text {mean }}, \mathrm{entr}_{\min / \max }$ & Normalized entropy & $\operatorname{entr}_{j}=\frac{-1}{\log n} \sum_{x \in \mathscr{U}} x_{j} \log x_{j}$ \\
\hline $\operatorname{corr}_{\text {min }}, \operatorname{corr}_{\text {max }}, \operatorname{corr}_{\text {mean }}, \operatorname{corr}_{\text {min } / \text { max }}$ & Correlation between features & $\operatorname{corr}_{j k}=\left(\sigma_{j}^{2} \cdot \sigma_{k}^{2}\right)^{-\frac{1}{2}} \sum_{x \in \mathscr{U}}\left(x_{j}-\mu_{j}\right)\left(x_{k}-\mu_{k}\right)$ \\
\hline skew $_{\min }$, skew $_{\max }$, skew $_{\text {mean }}$, skew $_{\min / \max }$ & Skewness & skew $_{j}=\frac{n}{(n-1)(n-2)} \sum_{x \in \mathscr{U}} \frac{\left(x_{j}-\mu_{j}\right)^{3}}{\sigma_{j}^{3}}$ \\
\hline $\mathrm{kurt}_{\text {min }}, \mathrm{kurt}_{\text {max }}, \mathrm{kurt}_{\text {mean }}, \mathrm{kurt}_{\text {min } / \text { max }}$ & Kurtosis & $\operatorname{kurt}_{j}=\left\{\frac{n(n+1)}{(n-1)(n-2)(n-3)} \sum_{x \in \mathscr{U}} \frac{\left(x_{j}-\mu_{j}\right)^{4}}{\sigma_{j}^{4}}\right\}-\frac{3(n-1)^{2}}{(n-2)(n-3)}$ \\
\hline $\operatorname{conn}_{k 1}, \operatorname{conn}_{k 1.5}, \operatorname{conn}_{k 2}$ & Connectivity $k$-means & Cluster validity measure (XU; WUNSCH, 2009). \\
\hline $\operatorname{conn}_{h 1}, \operatorname{conn}_{h 1.5}, \operatorname{conn}_{h 2}$ & Connectivity hierarc. clust. & Cluster validity measure (XU; WUNSCH, 2009). \\
\hline $\operatorname{dunn}_{k 1}$, dunn $_{k 1.5}$, dunn $_{k 2}$ & Dunn index $k$-means & Cluster validity measure (DUNN, 1974). \\
\hline $\operatorname{dunn}_{h 1}, \operatorname{dunn}_{h 1.5}$, dunn $_{h 2}$ & Dunn index hierarc. clust. & Cluster validity measure (DUNN, 1974). \\
\hline $\operatorname{silh}_{k 1}, \operatorname{silh}_{k 1.5}, \operatorname{silh}_{k 2}$ & Silhouette $k$-means & Cluster validity measure (ROUSSEEUW, 1987). \\
\hline $\operatorname{silh}_{h 1}, \operatorname{silh}_{h 1.5}, \operatorname{silh}_{h 2}$ & Silhouette hierarc. clust. & Cluster validity measure (ROUSSEEUW, 1987). \\
\hline
\end{tabular}

for a multi-label scenario. As mentioned in Section 4.2.6, we rounded the AUC performances to 2 significant digits. On the one hand, this leads to a more accurate analysis since we do not differentiate CPDP methods with very similar performances. On the other hand, however, this leads to a multi-label classification task since more than one label can be associated to the same meta-instance (see Section 2.7). Table 16 presents the best AUC performance obtained for each project and the respective best methods with equivalent performances.

For this multi-label classification task we applied the Binary Relevance (BR) transformation method (LUACES et al., 2012). As discussed in Section 2.7, this method creates $c$ distinct datasets ( $c=|\mathscr{L}|$, total number of labels), each for one of the four possible labels. The multi-label problem is then transformed in four binary classification problems. For each dataset $D_{\lambda_{j}}, 1 \leq j \leq c$, the target attribute is positive for meta-instances that belongs to the label $\lambda_{j}$ and negative otherwise.

\subsubsection{Meta-data Preprocessing}

In the preprocessing step, we address two issues. First, we mitigate the likely influence of the different ranges and scales of data over the meta-model performance. For this, we applied the z-score normalization (NAM; PAN; KIM, 2013). In this normalization technique, each meta-feature column is centered by subtracting the mean; and also scaled by dividing each value by the standard deviation. Second, we address the class-imbalance issue, possibly resulting from the BR transformation method (ZHANG; ZHOU, 2014). For example, consider the label 2009Turhan_nb. This method performed the best AUC for only $26 \%$ of all project datasets (see Table 16), which leads to an imbalanced binary dataset. In order to mitigate this issue, we applied the oversampling technique (GALAR et 
al., 2012). This technique consists of randomly duplicating instances of the minority class until the desired class distribution is achieved. It allows to adjust the class distribution of a dataset without discarding information. This characteristic is important in the context of this study considering the limited amount of meta-instances. On the other hand, the duplication of instances may lead to overfitting on data.

\subsubsection{Meta-learner}

Given a new software project dataset, represented by the meta-instance $\mathbf{x}_{i} \in \mathbb{R}^{m \times 1}$, the meta-model must be able to recommend an appropriate CPDP method (or label $\lambda \in \mathscr{L}$ for $\mathbf{x}_{i}$ ). For each dataset $D_{\lambda_{j}}, 1 \leq j \leq c$, generated with BR transformation method (see Section 5.2.3), we apply a binary classifier able to generate the confidence of relevance $C_{i j}$ (the confidence of $\lambda_{j}$ be a relevant label for $\mathbf{x}_{i}$ ). The final recommended label $\lambda$ refers to the label $\lambda_{j}$ with higher confidence of relevance $C_{i j}$.

For this task, we used the Random Forest algorithm (BREIMAN, 2001). Based on our experiments, this classifier presented the best learning capacity compared to each of the five classifiers investigated in this study. This algorithm is also a common choice in the contexts of software defect prediction (MALHOTRA, 2015) and algorithm recommendation (DORES et al., 2016; SANTOS; CARVALHO, 2016).

\subsubsection{Performance Measure}

Several performance measures focused on different aspects of multi-label learning have been proposed in the literature (WU; ZHOU, 2016). In this study we are specifically interested in evaluating the frequency (or accuracy) in which the top-ranked (higher confidence of relevance $C_{i j}$ ) label is actually among the relevant labels of an instance. This aspect can be obtained from the one-error measure (SCHAPIRE; SINGER, 2000). The one-error measures how many times the predicted label was not in $L_{i}$ (set of relevant labels for $\mathbf{x}_{i}$ ). It is defined as follows:

$$
\operatorname{one-error}(F)=\frac{1}{n} \sum_{i=1}^{n} \llbracket \arg \max F\left(x_{i}\right) \notin L_{i} \rrbracket \text {. }
$$

Note that, for single-label classification tasks, the one-error is identical to the ordinary error measure. Our general goal is to maximize the accuracy given by $\operatorname{acc}(F)=$ 1 - one-error $(F)$.

\subsubsection{Feature Selection}

As mentioned in Section 5.2.1, the amount of meta-data available is limited. High dimensional datasets, with redundant and irrelevant features, can lead to an ineffective 
performance (PARMEZAN; LEE; WU, 2017). Therefore, we also apply a feature selection method over the meta-data in order to achieve a suitable subset of meta-features.

We apply a Best-First Forward Wrapper strategy, adapted from Kalousis and Hilario (2001). This strategy applies an extensive and systematic search in the state space of all possible feature subsets using the Best-First heuristic (KOHAVI; SOMMERFIELD, 1995). The search is guided by the estimated accuracy of each subset, provided by an induction algorithm. To estimate the accuracy of a given subset we apply the CPLOO cross-validation; where each version (or meta-instance) is tested over a training set containing all the remaining project versions, excepting the versions of same project (see Section 3.4.3). The accuracy measure is presented in Section 5.2.6 and the induction algorithm is discussed in Section 5.2.5.

At the end, the subset of meta-features with highest accuracy is selected.

\subsection{Performance Evaluation}

In an ideal scenario, we would be able to construct a meta-model based on the 47 available datasets (as proposed in Section 5.2) and evaluate its performance with a different set of datasets. However, this scenario is not possible since this different set of datasets is not available. In order to approximate the ideal scenario, we evaluate the proposed solution based on a variation of the CPLOO procedure, here called meta-CPLOO. We adapted this leave-one-out procedure to the context of CPDP although it is a common approach in the meta-learning literature (DORES et al., 2016).

We separate one project (and its respective versions) for testing and construct the meta-model based on the remaining project versions. The meta-model is constructed following the configuration presented in the previous section. Finally, each version is tested separately with the respective constructed meta-model. It is important to observe that 15 different meta-models (one for each project) will be constructed to test all the 47 project versions. At the end, a CPDP method is recommended for each version.

On the one hand, the meta-CPLOO procedure enables us to estimate the performance for the proposed solution. On the other hand, it reduces the already limited amount of meta-data. For example, if we separate for test the five versions of the Ant project, the meta-model will be constructed based only on 42 versions. Consequently, the amount of available data for the internal CPLOO procedures are reduced.

Based on the meta-CPLOO procedure, we evaluate the performance of the proposed meta-learning solution in two different levels: meta-level and base-level. In the meta-level we evaluate the meta-learning capacity, i.e., whether the meta-learner can learn from the meta-data in relation to the defined baselines. In the base-level we evaluate the performance 
of the meta-learning solution across datasets in terms of AUC and compare it in relation to the four base CPDP methods. In each level, we evaluate two different configurations of meta-learning: MS-Dist and MS-Uns; referring to the two meta-features sets presented in Section 5.2.2.

\subsubsection{Meta-level}

In the meta-level analysis, we evaluate two sources of results. The first source is obtained from the feature selection step (see Section 5.2.7). For each of the 15 projects, the subset of features with highest accuracy estimate is selected to compose the meta-model. We compare these accuracy estimates in relation to a baseline, defined as the majority class. In this context, the majority class is given by the most frequent label of the respective meta-data. The statistical significance is verified with the non-parametric Wilcoxon signed rank test $(p$-value $\leq$ 0.05) (KEUNG; KOCAGUNELI; MENZIES, 2013).

The second source is obtained from the final recommendations provided by the proposed solution. We compare the accuracy (i.e., the rate of correct predictions) obtained with the meta-learning solution in relation to the majority class. In this context, the majority class is given by the most frequent label presented in Table 16 .

\subsubsection{Base-level}

In the base-level analysis, we compare the general performance (in terms of AUC) of the meta-learning solution in relation to the four considered CPDP methods applied individually. In addition, we compare all the performances in relation to a random baseline. For each project version, one of the four evaluated methods is randomly selected. This process is repeated 30 times. The random baseline is given by the mean AUC of the respective selected methods. The statistical analysis is based on the Friedman test followed by Fisher's LSD test (PEREIRA; AFONSO; MEDEIROS, 2015), as presented in Section 4.2 .6 .

\subsection{Results}

\section{RQ5.1 To what extent can meta-learning help us to select the most suitable CPDP method for a given dataset?}

RQ5.1.1 Does the meta-learner learn? (Meta-level)

To answer this question, we evaluate two sources of results, as discussed in Section 5.3.1. First, we compare the accuracies estimates produced in the feature selection step 
(see Section 5.2.7) for each of the 15 generated meta-models. We use these accuracies to evaluate the learning capacity of the two meta-learning configurations: MS-Dist and MS-Uns. We compare their performances in relation to the majority class baseline. The obtained accuracies are presented in Table 18.

Table 18 - The estimated accuracies and selected subsets for each meta-model. The majority class is used as a comparative reference.

\begin{tabular}{|c|c|c|c|c|c|}
\hline & & \multicolumn{2}{|c|}{ Best-First - Acc } & \multicolumn{2}{|c|}{ Best-First - Selected Subset } \\
\hline Project & Majority & MS-Dist & MS-Uns & MS-Dist & MS-Uns \\
\hline ant & 0.500 & 0.595 & 0.595 & $\mathrm{dam}_{m e d}, \mathrm{mfa}_{\text {med }}$ & $\operatorname{mean}_{\min / \max }$ \\
\hline camel & 0.465 & 0.628 & 0.628 & avg__cc $\mathrm{cc}_{s d}, \mathrm{cam}_{\text {med }}$ & $\operatorname{corr}_{\text {mean }}, \mathrm{kurt}_{\text {mean }}, \operatorname{silh}_{h 1}$ \\
\hline ckjm & 0.435 & 0.609 & 0.739 & $\max \_\mathrm{cc}_{\max }, \mathrm{avg} \_\mathrm{cc}_{s d}$ & $\mathrm{sd}_{\min / \max }, \operatorname{corr}_{\text {mean }}, \mathrm{dunn}_{k 1.5}, \operatorname{silh}_{k 1.5}$ \\
\hline forrest & 0.533 & 0.733 & 0.733 & $\mathrm{npm}_{s d}, \mathrm{rfc}_{m e d}, \mathrm{ce}_{\text {med }}$ & $\operatorname{corr}_{\text {mean }}$, skew $_{\text {min }}$, dunn $_{h 1.5}, \operatorname{silh}_{k 1.5}$ \\
\hline ivy & 0.432 & 0.636 & 0.568 & $\operatorname{mfa}_{\max }$ & $\mathrm{sd}_{\min / \max }, \operatorname{silh}_{k 1.5}$ \\
\hline jedit & 0.405 & 0.548 & 0.500 & $\mathrm{ce}_{\text {min }}, \mathrm{npm}_{s d}$ & $\operatorname{dunn}_{k 1.5}$ \\
\hline $\log 4 j$ & 0.432 & 0.568 & 0.659 & $\mathrm{rfc}_{\text {med }}, \mathrm{ic}_{\text {med }}$ & skew $_{\text {mean }}$, kurt $_{\text {mean }}, \operatorname{silh}_{k 1.5}$ \\
\hline lucene & 0.409 & 0.545 & 0.591 & avg__cc $\mathrm{cc}_{\text {med }}$ & $\mathrm{sd}_{\min / \max }$, skew $_{\min }, \operatorname{conn}_{k 2}$ \\
\hline pbeans & 0.444 & 0.533 & 0.622 & $\mathrm{dit}_{\text {min }}$ & skew $_{\text {mean }}, \operatorname{silh}_{h 2}, \operatorname{silh}_{k 1.5}$ \\
\hline poi & 0.442 & 0.698 & 0.535 & $\max \_\mathrm{cc}_{\max }, \mathrm{dit}_{\min }$, avg__ $\mathrm{cc}_{s d}$ & $\operatorname{mean}_{\min / \max }$ \\
\hline synapse & 0.477 & 0.682 & 0.636 & avg_cc $c_{s d}$, moa $_{m e d}, \max \_\mathrm{cc}_{m e d}$ & $\mathrm{sd}_{\min / \text { max }}, \operatorname{corr}_{\text {mean }}, \mathrm{skew}_{\text {mean }}, \operatorname{silh}_{k 1.5}$ \\
\hline tomcat & 0.457 & 0.674 & 0.587 & $\mathrm{npm}_{\max }, \operatorname{loc}_{\min }$, avg_ccc $\mathrm{cc}_{s d}$ & $\operatorname{silh}_{k 1.5}$ \\
\hline velocity & 0.477 & 0.614 & 0.523 & $\mathrm{cbm}_{\max }, \mathrm{moa}_{s d}, \mathrm{ca}_{\text {med }}$ & $\operatorname{corr}_{\max }$ \\
\hline xalan & 0.395 & 0.674 & 0.488 & $\mathrm{amc}_{\text {max }}, \mathrm{lcom}_{\text {mean }}, \mathrm{rfc}_{\text {sd }}$, avg_cc $\mathrm{cc}_{\text {med }}$ & kurt $_{\text {mean }}$ \\
\hline xerces & 0.465 & 0.698 & 0.628 & $\operatorname{loc}_{\text {min }}, \mathrm{cam}_{\text {min }}, \mathrm{ca}_{\text {mean }}$, avg_cc $\mathrm{cc}_{\text {med }}$ & $\mathrm{sd}_{\text {min/max }}, \mathrm{kurt}_{\text {mean }}, \operatorname{silh}_{k 1.5}$ \\
\hline Mean & 0.451 & 0.629 & 0.602 & - & - \\
\hline
\end{tabular}

Initially, we can observe that both meta-features sets $M S$-Uns and MS-Dist produced an accuracy superior to the majority class for all projects meta-data. $M S$-Dist produced the best mean value although it can not be differentiated from $M S$-Uns with statistical significance. These performances are better visualized in Figure 18.

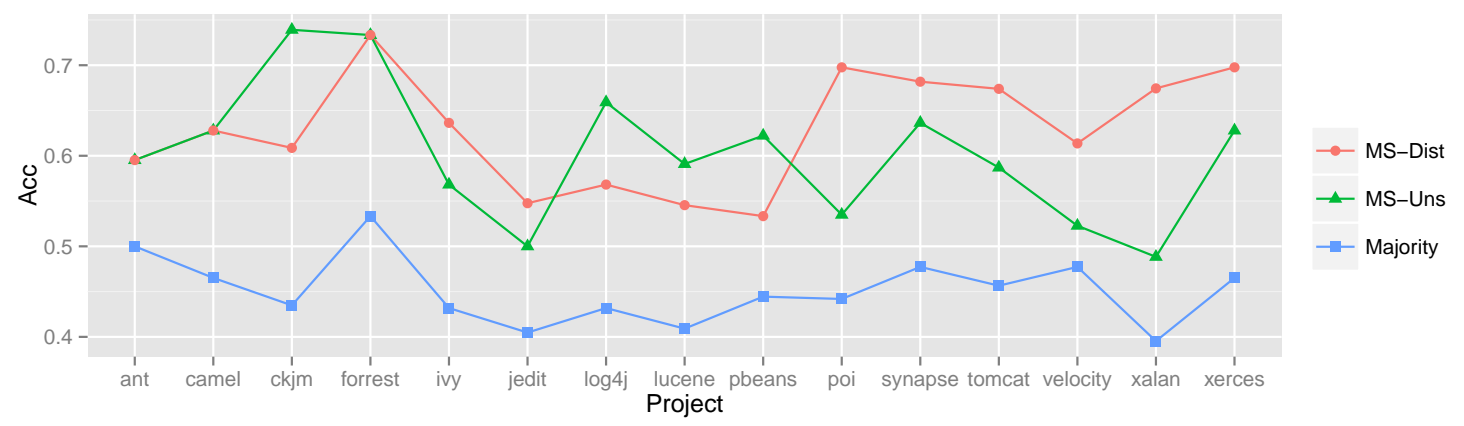

Figure 18 - The resulting best accuracies generated for each meta-model in the feature selection step. The majority class is used as a comparative reference for the meta-features sets MS-Dist and MS-Uns.

Although the obtained performances characterize some level of meta-learning capacity, they can also represent an overfitting on data; i.e., when a subset achieves a high accuracy estimate but poor predictive power for new instances (KOHAVI; SOMMERFIELD, 1995). For small samples and a high dimensionality of features (such as the evaluated context), it is likely that one of the many feature subsets lead to a hypothesis with high predictive accuracy (KOHAVI; SOMMERFIELD, 1995). 
The existence of overfitting can be verified by testing the meta-models for new instances. For this, we use each meta-model to recommend a suitable CPDP method for the respective project versions previously separated for test (see Section 5.3). The meta-learners $M S$-Dist and $M S$-Uns recommended correctly a suitable CPDP method for $25(53 \%)$ and $16(34 \%)$ of the 47 tested project versions, respectively. The majority class, represented by the label 2013He_rf, achieved the best AUC for 20 (43\%) of all project versions. These results indicate an appropriate learning of the meta-learning solution based on the meta-features set $M S$-Dist since it produced an accuracy superior to the majority class, defined as the baseline.

RQ5.1.2 How does the meta-learner perform across datasets? (Base-level)

The general performance of the meta-learning solution is discussed below. We compare the resulting recommendations provided in the meta-CPLOO procedure in relation to the four evaluated CPDP methods applied individually. We also compare the meta-learning performances with the random baseline. Table 19 presents the mean ranking and mean AUC values for each method as well as the frequency in which each method performed the best and worst AUC value for a project among the four possible labels.

Table 19 - The general performance of the proposed meta-learning solution for both metafeatures sets. These performances are compared with the four base CPDP methods and the random baseline.

\begin{tabular}{lcccc}
\hline Method & Mean Rank & Mean AUC & Freq. Best & Freq. Worst \\
\hline Meta_MS-Dist & $\mathbf{3 . 4 0}( \pm 1.86)$ & $\mathbf{0 . 7 7 4}( \pm 0.10)$ & $\mathbf{2 5} \mathbf{5 3 \%})$ & $\mathbf{1 2}(\mathbf{2 6 \%})$ \\
\hline 2012Ma_nb & $3.61( \pm 1.88)$ & $0.771( \pm 0.10)$ & $19(40 \%)$ & $12(26 \%)$ \\
\hline 2013He_rf & $3.76( \pm 1.83)$ & $0.766( \pm 0.09)$ & $20(43 \%)$ & $11(23 \%)$ \\
\hline Meta_MS-Uns & $\mathbf{4 . 0 3}( \pm 1.81)$ & $\mathbf{0 . 7 7 0}( \pm 0.09)$ & $\mathbf{1 6}(\mathbf{3 4 \%})$ & $\mathbf{1 7}(\mathbf{3 6 \%})$ \\
\hline Random & $\mathbf{4 . 3 2}( \pm 0.92)$ & $\mathbf{0 . 7 6 6}( \pm 0.09)$ & $\mathbf{4}(\mathbf{9 \%})$ & $\mathbf{3}(\mathbf{6 \%})$ \\
\hline 2013He_svm & $4.37( \pm 1.95)$ & $0.765( \pm 0.09)$ & $13(28 \%)$ & $18(38 \%)$ \\
\hline 2009Turhan_nb & $4.51( \pm 2.13)$ & $0.761( \pm 0.09)$ & $12(26 \%)$ & $21(45 \%)$ \\
\hline
\end{tabular}

The meta-learner $M S$-Dist presented the best mean ranking and highest mean AUC of all evaluated methods. This solution also presented the highest frequency of best AUC. It provided the best AUC performance for $53 \%$ of all project versions against $40 \%$ of the second best-ranked method (2012Ma_nb). Together with 2012Ma_nb, the solution $M S$-Dist provided the worst AUC performance for only $26 \%$ of all project versions. It is worth to note that even the worst AUC performance represents one of the four best ranked solution across datasets, as discussed in Section 5.2.3.

We applied the Friedman test and obtained the $p$-value $=0.044$, which refuses the null hypothesis of performance equivalence between methods. The results of the Fisher's LSD test are presented in Figure 19. From these results, we can highlight: 1) the random baseline is not significantly different from 2013He_svm and 2009Turhan_nb; 2) the meta-learner $M S$-Uns can not be differentiated from the random baseline; 3) the three 
best-ranked methods MS-Dist, 2012Ma_nb, and 2013He_rf present significant difference in relation to the random baseline; and 4) the two best-ranked methods do not present significant difference from each other although $M S$-Dist present higher mean $A U C$ and higher frequency of best AUC performance.

$\begin{array}{lllllll}\text { Meta_MS-Dist } & \text { a } & & & & \\ \text { 2012Ma_nb } & \text { a } & \text { b } & & & \\ \text { 2013He_rf } & & \text { b } & c & & \\ \text { Meta_MS-Uns } & & & c & d & \\ \text { Random } & & & & d & \text { e } \\ \text { 2013He_svm } & & & & & \text { e } \\ \text { 2009Turhan_nb } & & & & & \text { e }\end{array}$

Figure 19 - Pairwise Post-hoc Fisher's LSD test. The meta-learner MS-Dist presents the best ranking position along with the base method 2012Ma_nb, with no significant difference.

\subsection{Threats to Validity}

The experiments conducted in this chapter make use of the experimental resources presented in Chapter 4. Therefore, the threats to validity presented in Section 4.4 are still valid in this context.

Another important issue, specific for the experiments conducted in this chapter, regards to the CPDP methods considered as the labels for the meta-target. Those CPDP methods presented the best ranking performances across datasets. Also, they presented distinct inductive bias in relation to each other, which can contribute both for the diversity as well as the complementarity of the meta-learning solution. However, other criteria can be considered in this case, which may completely alter the obtained conclusions.

In addition, different approaches for the internal procedures of the proposed solution can lead to different results. For example, different meta-features or a different feature selection method can be used. These issues should be investigated in future work.

\subsection{Conclusion}

In this chapter we proposed an automatic resource for the recommendation of suitable CPDP methods according to the characteristics of the software being predicted. The proposed solution is based on the general architecture of meta-learning for algorithm recommendation. Several particularities inherent to the context of CPDP were addressed, including the unsupervised characterization of datasets, the multi-label meta-target, the modified cross-validation procedure, and the evaluation procedure.

We evaluated two distinct meta-features sets. The meta-learning solution presented better performance when configured with the $M F$-Dist set, defined by distributional 
metrics applied over the dataset features. The MF-Dist meta-learner presented a better performance in relation to the four evaluated CPDP methods considering the frequency of predicted best solutions. In other words, the meta-learner was able to predict the best solution for a larger amount of project versions. This result indicates a proper predictive power of the proposed solution in the meta-level. In the base-level, however, even though the meta-learner presented the higher value of mean AUC, it did not present significant difference in relation to the second best ranked CPDP method.

The results are positive and reveal a proper learning of the proposed solution. In the next chapter we discuss the general conclusions, highlight the main contributions presented in this thesis and sketch the future work. 
CHAPTER

6

10

\section{CONCLUSION}

In this thesis, we aimed at providing alternative solutions for CPDP and to provide support for test practitioners on selecting an appropriate CPDP method.

First, we proposed that the performance of CPDP instance filtering methods can be improved by selecting only the most relevant features for the calculation of similarity between training and test instances. We evaluated this proposal for both prediction contexts - classification and ranking. In total, we evaluated 17 methods derived from different configurations of four distinct feature selection methods applied over 36 versions of 11 open source software projects. The results show that for all the analyzed projects at least one of the 17 evaluated methods presented better performance in relation to the original filtering methods. We also evaluated which methods present better performance. We identified that no evaluated method presented best performance for all datasets, i.e, the most appropriate method can vary for each project.

Then, we conducted an experimental comparison of 31 CPDP methods derived from six state-of-the-art transfer learning solutions associated to the five most frequently used classifiers in the defect prediction literature. Lastly, we investigated the feasibility of using meta-learning for the automatic recommendation of suitable CPDP methods according to the characteristics of the software being predicted.

These experiments were based on 47 versions of 15 open source software projects. Different from previous studies in the literature, we considered an application domain in which no previous information about the software is demanded. In practice, this characteristic allows a broader applicability of defect prediction models and the proposed meta-learning solution. For example, for companies with insufficient data from previous projects or for projects in its first release. This characteristic is possible due to three main factors: 1) the training set is composed of known external projects; 2) the software characterization can be extracted directly from the source code; and 3) the meta-model is 
constructed based on unsupervised meta-features extracted from the target set.

From the performance comparison experiment, we identified the four best-ranked CPDP methods across datasets in terms of AUC: 2012Ma_nb, 2013He_rf, 2009Turhan_nb, and $2013 H e \_s v m$. These four methods, however, presented the best performance for distinct groups of datasets. In other words, the most suitable CPDP method for a project varies according to the project being predicted. These results accredited the investigation of the proposed meta-learning solution.

We evaluated two distinct unsupervised meta-features sets for a multi-label metalearning task. The performance analysis was conducted in two levels. In the meta-level, the results indicate a proper predictive power of the proposed solution. The meta-learner based on the meta-features set $M S$-Dist presented an accuracy of 10 percentage points superior to the majority class. In the base-level, we compared the proposed solution in relation to the four best-ranked CPDP methods across datasets in terms of AUC. Although there is no significant difference in relation to the second best-ranked method 2012Ma_nb, the meta-learner $M S$-Dist provided the higher mean AUC.

Assuming the proper generalization of these results, three factors contribute to the practical use of the proposed meta-learning solution: 1) there is learning in the meta-level. The meta-learner provided the best solution for a larger amount of datasets than each of the four evaluated methods applied individually; 2) in the worst case, the meta-learner will still recommend one of the four CPDP methods with best ranking across datasets; and 3) considering its application domain, the proposed solution is not expensive. The hard computational cost is spent in the meta-model construction. For the recommendation task, only the meta-characterization and prediction costs are demanded.

\subsection{Contributions}

The contributions of this thesis can be divided into three groups, concentrated in the Chapters 3, 4, and 5 .

In Chapter 3, we investigated the feasibility of using feature selection methods as an internal procedure to improve the performance of two instance filtering methods. Five main contributions can be highlighted: 1) we proposed the use of feature selection methods to select the most relevant features for the calculation of the Euclidean distance between training and test instances; 2) we proposed an appropriate experimental setup, including the data collection, software metrics, experiment design, feature selection methods, prediction model, and performance measures; 3) we proposed the performance evaluation in both contexts - classification and ranking; 4) we managed to identify the feasibility of the proposed solution; and 5) we managed to identify the most frequently best IFFS methods. 
The contributions presented in this chapter were initially published in Porto and Simao (2016b). The published paper was selected among the three best ranked papers and invited to submit an extended version for the CLEI Electronic Journal (PORTO; SIMAO, 2016a).

In Chapter 4, we provided an experimental performance evaluation of the stateof-the-art CPDP methods. Four main contributions can be highlighted: 1) we provided the first extensive performance comparison of CPDP methods on the literature; 2) we provided a uniform experimental setup, including the collection of data, preprocessing resources, statistical analysis, performance measure, and methodology; 3) we managed to identify the four CPDP methods with most frequently best performance across datasets; and 4) we managed to distinguish the groups of datasets in which each of the four methods perform best.

In Chapter 5, we investigated the feasibility of using meta-learning for the recommendation of suitable CPDP methods according to the software characteristics. Five main contributions can be highlighted: 1) we proposed a meta-learning architecture applied to CPDP; 2) we proposed to use unsupervised meta-features for the characterization of target datasets; 3) we proposed an appropriate methodology considering the inherent particularities of the investigated context, including the meta-features sets, the multi-label meta-target, the meta-data preprocessing, the multi-label meta-learner, the multi-label performance measure, and the wrapper feature selection method; 4) we defined an appropriate methodology for the evaluation of the proposed solution encompassing two levels with the appropriate statistical analysis; and 5) we managed to identify the learning capacity of the proposed solution and its performance in relation to the base methods.

The contributions presented in Chapters 4 and 5 were transcribed in an article and submitted to the IEEE Transactions on Software Engineering (TSE) Journal.

\subsection{Limitations and Future Work}

Despite the positive contributions presented in this thesis, we also identified some limitations and open issues to be investigated in future work.

The research conducted in Chapter 3 can be continued. The obtained results accredit the investigation of automated resources for the recommendation of the most appropriate IFFS method to be applied according to the project characteristics. A meta-learning solution can be adapted from the concepts proposed in Chapter 5 and applied for the recommendation of IFFS methods.

As discussed in Section 3.6, the quality of the results presented in this thesis is dependent on the quality of the data collection used in the experiments. These data were 
collected following a systematic procedure and were largely replicated in the literature (ZIMMERMANN; PREMRAJ; ZELLER, 2007; ZHANG et al., 2014; MADEYSKI; JURECZKO, 2014). However, there is no guarantee that the number of defects identified for each software part represents the actual number of existing defects. Nevertheless, this guarantee is difficult (if not impossible) to acquire (YANG; TANG; YAO, 2015; KITCHENHAM; MENDES; TRAVASSOS, 2007). In addition, the data collection is composed of only Java open source software projects, which limits the generalization of the obtained results. Other data collections with different characteristics and sources can be investigated and summed with the results presented in this thesis.

The experimental comparison presented in Chapter 4 is composed of 31 CPDP methods. Even though it provides a representative sample of the state-of-the-art in CPDP, it does not represent an exhaustive comparison of all the existent methods or the most recent methods in the literature of $\mathrm{CPDP}$. This research can be extended covering a different sample of CPDP methods.

In the experiments conducted in Chapter 4, we also limited the set of metrics to the code metrics only. We used only this set of metrics since the number of features can strongly influence the performance of the base classifiers due to their inherent inductive biases. For example, the Random Forest algorithm is more robust to redundant and irrelevant features than the Naïve Bayes algorithm. Another limitation refers to the base classifiers used in the conducted experiments. Other criteria instead of the most frequently used classifiers can lead to a different experimentation setup and results.

Further studies are still necessary to guarantee the generalization of the presented results. In addition, alternative solutions can be investigated aiming at improving the meta-learning performance. We point out three factors for future investigation. First, the meta-data can be expanded. A larger amount of meta-instances can contribute to improve the predictive power of the meta-learner. Second, other meta-features sets can be explored. For example, the relation (or difference) between testing and training data can be considered in conjunction with the particularities of each target set. Third, other feature selection methods can lead to subsets with higher predictive power.

Other applications and approaches for meta-learning can also be explored in the context of CPDP. For example, the meta-target can be configured to a regression task (KANDA et al., 2016). In this approach, a ranking of suitable solutions for a given domain is provided. The ranking information can be explored in at least three ways: 1) the $N$ best ranked CPDP methods can be selected to compose an ensemble solution (CRUZ; SABOURIN; CAVALCANTI, 2017); 2) the ordered list of CPDP methods can be interpreted as weights in a weighted majority vote for an ensemble solution (KUNCHEVA, 2004); or 3) an hybrid solution from 1) and 2) considering the $N$ best ranked methods in a weighted majority vote scheme (CRUZ; SABOURIN; CAVALCANTI, 2017). 
In this thesis, we focused on the classification task of defect prediction models. However, other tasks can be explored. The ranking task was investigated in Chapter 3. This same task can be investigated in the context of the experiments conducted in Chapters 4 and 5 . 

AKIYAMA, F. An example of software system debugging. In: IFIP Congress (1). [S.l.: s.n.], 1971. p. 353-359. Citation on page 39.

ALI, S.; SMITH, K. A. On learning algorithm selection for classification. Applied Soft Computing, v. 6, n. 2, p. 119 - 138, 2006. ISSN 1568-4946. Available: <http://dx.doi. org/10.1016/j.asoc.2004.12.002>. Citations on pages 28 e 50.

ANDRIOLE, S. J. Software validation, verification, testing and documentation: A source book. Princeton, NJ, USA: Petrocelli Books, Inc., 1986. Citation on page 34.

BACCHELLI, A.; D’AMBROS, M.; LANZA, M. Are popular classes more defect prone? In: Proc. of the 13th Int. Conf. on Fund. Appr. to Soft. Eng. Springer-Verlag, 2010. (FASE'10), p. 59-73. ISBN 3-642-12028-8, 978-3-642-12028-2. Available: <http: //dx.doi.org/10.1007/978-3-642-12029-9_5>. Citation on page 40.

BASILI, V.; BRIAND, L.; MELO, W. A validation of object-oriented design metrics as quality indicators. IEEE Trans Softw Eng, v. 22, p. 751-761, 1996. Available: <http://dx.doi.org/10.1109/32.544352>. Citation on page 40.

BEIZER, B. Software Testing Techniques. 2nd. ed. [S.l.]: Van Nostrand Reinhold Co., 1990. ISBN 0-442-20672-0. Citation on page 35.

BELL, R. M.; WEYUKER, E. J.; OSTRAND, T. J. Assessing the impact of using fault prediction in industry. In: 2011 IEEE Fourth International Conference on Software Testing, Verification and Validation Workshops. [s.n.], 2011. p. 561-565. Available: <http://dx.doi.org/10.1109/ICSTW.2011.75>. Citations on pages 26 e 41.

BIRD, C.; NAGAPPAN, N.; MURPHY, B.; GALL, H.; DEVANBU, P. Don't touch my code!: Examining the effects of ownership on software quality. In: Proc. of the 19th ACM SIGSOFT Symp. and the 13th Eur. Conf. on Found. of Soft. Eng. ACM, 2011. (ESEC/FSE '11), p. 4-14. ISBN 978-1-4503-0443-6. Available: <http://dx.doi.org/ $10.1145 / 2025113.2025119>$. Citation on page 40.

BOEHM, B.; BASILI, V. R. Software defect reduction top 10 list. Computer, v. 34, n. 1, p. 135-137, Jan. 2001. ISSN 0018-9162. Available: <http://dx.doi.org/10.1109/2.962984>. Citations on pages 25 e 34 .

BOEHM, B. W.; BROWN, J. R.; KASPAR, H. Characteristics of Software Quality. Amsterdam: North-Holland, 1978. Citations on pages 17, 33 e 34.

BRAZDIL, P.; GIRAUD-CARRIER, C.; SOARES, C.; VILALTA, R. Metalearning: Applications to Data Mining. Springer Berlin Heidelberg, 2009. ISBN 9783540732624. Available: <http://dx.doi.org/10.1007/978-3-540-73263-1>. Citation on page 95.

BRAZDIL, P.; VILALTA, R.; GIRAUD-CARRIER, C.; SOARES, C. Metalearning. In: Encyclopedia of Machine Learning and Data Mining. Boston, MA: Springer 
US, 2017. p. 818-823. ISBN 978-1-4899-7687-1. Available: <http://dx.doi.org/10.1007/ 978-1-4899-7687-1_543>. Citations on pages 27, 42, 50, 51, 52, 93 e 95.

BRAZDIL, P. B.; HENERY, R. J. Machine learning, neural and statistical classification. In: . New York: Ellis Horwood, 1994. chap. Analysis of Results, p. 175-212. Citations on pages 28 e 51.

BRAZDIL, P. B.; SOARES, C.; COSTA, J. P. da. Ranking learning algorithms: Using ibl and meta-learning on accuracy and time results. Machine Learning, v. 50, n. 3, p. 251-277, Mar 2003. ISSN 1573-0565. Available: <https://doi.org/10.1023/A: 1021713901879>. Citations on pages 28 e 50.

BREIMAN, L. Random forests. Machine Learning, v. 45, n. 1, p. 5-32, 2001. Available: <http://dx.doi.org/10.1023/A:1010933404324>. Citations on pages 45, 65 e 98.

BRIAND, L. C.; MELO, W. L.; WUST, J. Assessing the applicability of fault-proneness models across object-oriented software projects. IEEE Trans. Softw. Eng., v. 28, n. 7, p. 706-720, Jul. 2002. ISSN 0098-5589. Available: <http://dx.doi.org/10.1109/TSE.2002. 1019484>. Citation on page 46.

BRODLEY, C. E.; SMYTH, P. Applying classification algorithms in practice. Statistics and Computing, v. 7, n. 1, p. 45-56, Mar 1997. ISSN 1573-1375. Available: <http: //dx.doi.org/10.1023/A:1018557312521>. Citation on page 27.

CHIDAMBER, S. R.; KEMERER, C. F. A metrics suite for object oriented design. IEEE Trans. Softw. Eng., IEEE Press, v. 20, n. 6, p. 476-493, Jun. 1994. ISSN 0098-5589. Available: <http://dx.doi.org/10.1109/32.295895>. Citation on page 40.

COVER, T. M.; THOMAS, J. A. Elements of Information Theory. New York: WileyInterscience, 1991. ISBN 0471241954. Citations on pages 56, 58 e 61.

CRUZ, A. E. C.; OCHIMIZU, K. Towards logistic regression models for predicting fault-prone code across software projects. In: Proc. Int. Symp. on Emp. Soft. Eng. and Meas. (ESEM '09). Washington, DC,USA: [s.n.], 2009. p. 460-463. Available: <http://dx.doi.org/10.1109/ESEM.2009.5316002>. Citations on pages 27, 47, 77 e 80.

CRUZ, R. M.; SABOURIN, R.; CAVALCANTI, G. D. Meta-des.oracle: Meta-learning and feature selection for dynamic ensemble selection. Information Fusion, v. 38, p. 84 - 103, 2017. ISSN 1566-2535. Available: <https://doi.org/10.1016/j.inffus.2017.02.010>. Citations on pages 51 e 108.

CSARDI, G.; NEPUSZ, T. The igraph software package for complex network research. InterJournal, Complex Systems, p. 1695, 2006. Citation on page 61.

D‘AMBROS, M.; LANZA, M.; ROBBES, R. Evaluating defect prediction approaches: a benchmark and an extensive comparison. Emp. Soft. Eng., v. 17, n. 4, p. 531-577, Aug 2012. ISSN 1573-7616. Available: <http://dx.doi.org/10.1007/s10664-011-9173-9>. Citation on page 40.

DEMSAR, J. Statistical comparisons of classifiers over multiple data sets. J. Mach. Learn. Res., JMLR.org, v. 7, p. 1-30, Dec. 2006. ISSN 1532-4435. Citation on page 84. 
DENARO, G.; PEZZè, M. An empirical evaluation of fault-proneness models. In: Proc Int Conf on Soft Eng (ICSE2002). [s.n.], 2002. p. 241-251. Available: <http://dx.doi. org/10.1145/581339.581371>. Citations on pages 40 e 55 .

DORES, S. Nunes das; ALVES, L.; RUIZ, D. D.; BARROS, R. C. A meta-learning framework for algorithm recommendation in software fault prediction. In: Proc. of Annual ACM Symp. on Appl. Comp. (SAC '16). New York, NY, USA: ACM, 2016. p. 1486-1491. ISBN 978-1-4503-3739-7. Available: <http://doi.acm.org/10.1145/2851613. 2851788>. Citations on pages 28, 51, 52, 64, 93, 95, 98 e 99.

DUNN, J. C. A fuzzy relative of the ISODATA process and its use in detecting compact well-separated clusters. Journal of Cybernetics, v. 3, p. 32-57, 1974. Citation on page 97.

ENGSTRÖM, E.; RUNESON, P.; WIKSTRAND, G. An empirical evaluation of regression testing based on fix-cache recommendations. In: Third Int. Conf. on Soft. Test., Verif. and Valid. [s.n.], 2010. p. 75-78. ISSN 2159-4848. Available: <http://dx.doi.org/10.1109/ ICST.2010.40>. Citations on pages 26 e 41.

FENTON, N. E.; NEIL, M. A critique of software defect prediction models. IEEE Trans Softw Eng, IEEE Press, Piscataway, NJ, USA, v. 25, n. 5, p. 675-689, Sep. 1999. Available: $<$ http://dx.doi.org/10.1109/32.815326>. Citation on page 39 .

FUKUSHIMA, T.; KAMEI, Y.; MCINTOSH, S.; YAMASHITA, K.; UBAYASHI, N. An empirical study of just-in-time defect prediction using cross-project models. In: Proc. of the 11th Work. Conf. on Mining Soft. Repos. ACM, 2014. (MSR 2014), p. 172-181. ISBN 978-1-4503-2863-0. Available: <http://dx.doi.org/10.1145/2597073.2597075>. Citation on page 40.

GALAR, M.; FERNANDEZ, A.; BARRENECHEA, E.; BUSTINCE, H.; HERRERA, F. A review on ensembles for the class imbalance problem: Bagging-, boosting-, and hybrid-based approaches. IEEE Tran. on Sys., Man, and Cyber., Part C (Applications and Reviews), v. 42, n. 4, p. 463-484, July 2012. ISSN 1094-6977. Available: <http://dx.doi. org/10.1109/TSMCC.2011.2161285>. Citation on page 98.

GAO, K.; KHOSHGOFTAAR, T. M. A comprehensive empirical study of count models for software fault prediction. IEEE Trans on Reliability, v. 56, n. 2, p. 223-236, 2007. Available: <http://dx.doi.org/10.1109/TR.2007.896761>. Citations on pages 38 e 40.

GHOTRA, B.; MCINTOSH, S.; HASSAN, A. E. Revisiting the impact of classification techniques on the performance of defect prediction models. In: 2015 IEEE/ACM 37th IEEE Int. Conf. on Soft. Eng. [s.n.], 2015. v. 1, p. 789-800. ISSN 0270-5257. Available: <https://doi.org/10.1109/ICSE.2015.91>. Citations on pages 26 e 41.

GUNARATHNA, D.; TURHAN, B.; HOSSEINI, S. A Systematic Literature Review on Cross-project Defect Prediction. Master's Thesis (Master's Thesis) — University of Oulu - Information Processing Science, Oct. 2016. Citations on pages 26, 27, 46, 48, 64, 77 e 83 .

HALSTEAD, M. H. Elements of Software Science (Operating and Programming Systems Series). New York, NY, USA: Elsevier Science Inc., 1977. ISBN 0444002057. Citation on page 39 . 
HASSAN, A. E. Predicting faults using the complexity of code changes. In: IEEE Int Conf on Softw Eng. IEEE, 2009. p. 78-88. Available: <http://dx.doi.org/10.1109/ICSE. 2009.5070510>. Citation on page 40 .

HAYKIN, S. Neural Networks: A Comprehensive Foundation. 2nd. ed. [S.l.]: Prentice Hall PTR, 1998. ISBN 0132733501. Citation on page 43.

HE, P.; LI, B.; LIU, X.; CHEN, J.; MA, Y. An empirical study on software defect prediction with a simplified metric set. Inf. and Soft. Tech., v. 59, p. $170-190,2015$. ISSN 0950-5849. Available: <https://doi.org/10.1016/j.infsof.2014.11.006>. Citation on page 49 .

HE, Z.; PETERS, F.; MENZIES, T.; YANG, Y. Learning from open-source projects: An empirical study on defect prediction. In: ESEM. IEEE Computer Society, 2013. p. 45-54. Available: <http://dx.doi.org/10.1109/ESEM.2013.20>. Citations on pages 27, 47, 48, $52,74,77,78,81,87$ e 96.

HE, Z.; SHU, F.; YANG, Y.; LI, M.; WANG, Q. An investigation on the feasibility of cross-project defect prediction. Automated Software Engineering, Kluwer Academic Publishers, Hingham, MA, USA, v. 19, n. 2, p. 167-199, Jun. 2012. Available: < http: //dx.doi.org/10.1007/s10515-011-0090-3>. Citations on pages 52 e 96.

HEARST, M. A. Trends controversies: Support vector machines. IEEE Intelligent System, v. 13, n. 4, p. 18-28, 1998. Citations on pages 42 e 43.

HERBOLD, S. Training data selection for cross-project defect prediction. In: Proc. Int. Conf. on Pred. Mod. in Soft. Eng. (PROMISE '13). New York, NY, USA: [s.n.], 2013. p. 6:1-6:10. Available: <http://dx.doi.org/10.1145/2499393.2499395>. Citations on pages $27,47,48,49,52,74,77,80,81,90$ e 96.

HERBOLD, S.; TRAUTSCH, A.; GRABOWSKI, J. Global vs. local models for crossproject defect prediction. Emp. Soft. Engin., p. 1-37, 2016. ISSN 1573-7616. Available: <http://dx.doi.org/10.1007/s10664-016-9468-y>. Citations on pages 26, 27, 48, 77 e 84.

HERZIG, K.; JUST, S.; RAU, A.; ZELLER, A. Predicting defects using change genealogies. In: IEEE Int Symp on Soft Reliab Eng 2013. IEEE, 2013. Available: <http://dx. doi.org/10.1109/ISSRE.2013.6698911>. Citations on pages 17, 26, 40, 55, 60, 63 e 65 .

IEEE. IEEE Standard Glossary of Software Engineering Terminology. 1990. 610.12-1990, IEEE. Citations on pages 34 e 36.

ISO/IEC. ISO/IEC 25010 - Systems and software engineering - Systems and software Quality Requirements and Evaluation (SQuaRE) - System and software quality models. [S.1.], 2010. Citation on page 33.

JAIN, A. K. Data clustering: 50 years beyond k-means. Pattern Recognition Letters, v. 31, n. 8, p. 651 - 666, 2010. Available: <http://dx.doi.org/10.1016/j.patrec.2009.09.011>. Citation on page 57 .

JAPKOWICZ, N.; SHAH, M. Evaluating Learning Algorithms: A Classification Perspective. [S.1.]: Cambridge University Press, 2011. ISBN 1107653118. Citation on page 65 . 
JIANG, T.; TAN, L.; KIM, S. Personalized defect prediction. In: 2013 28th IEEE/ACM International Conference on Automated Software Engineering (ASE). [s.n.], 2013. p. 279-289. Available: <http://dx.doi.org/10.1109/ASE.2013.6693087>. Citation on page 41.

JIANG, Y.; CUKIC, B.; MA, Y. Techniques for evaluating fault prediction models. Emp Softw Eng, v. 13, p. 561-595, 2008. Available: <http://dx.doi.org/10.1007/ s10664-008-9079-3>. Citations on pages 65 e 74.

JONES, C.; BONSIGNOUR, O. The Economics of Software Quality. 1st. ed. [S.l.]: Addison-Wesley Professional, 2011. ISBN 0132582201, 9780132582209. Citations on pages 25 e 37.

JURECZKO, M.; MADEYSKI, L. Towards identifying software project clusters with regard to defect prediction. In: Proc Int Conf on Pred Mod in Soft Eng (PROMISE2010). ACM, 2010. p. 9:1-9:10. Available: <http://dx.doi.org/10.1145/1868328.1868342>. Citations on pages $17,47,56,59,62,73,74$ e 79 .

KALOUSIS, A. Algorithm Selection via Meta-Learning. Phd Thesis (PhD Thesis), Geneva, 2002. Citations on pages 28, 50 e 93.

KALOUSIS, A.; HILARIO, M. Feature selection for meta-learning. In: Proc. of PacificAsia Conf. on Know. Disc. and Data Min. (PAKDD2001). London, UK, UK: Springer-Verlag, 2001. p. 222-233. ISBN 3-540-41910-1. Citation on page 99.

KAMEI, Y.; SHIHAB, E.; ADAMS, B.; HASSAN, A. E.; MOCKUS, A.; SINHA, A.; UBAYASHI, N. A large-scale empirical study of just-in-time quality assurance. IEEE Trans. on Soft. Eng., v. 39, n. 6, p. 757-773, June 2013. ISSN 0098-5589. Available: $<$ http://dx.doi.org/10.1109/TSE.2012.70>. Citation on page 40.

KANDA, J.; CARVALHO, A. de; HRUSCHKA, E.; SOARES, C.; BRAZDIL, P. Metalearning to select the best meta-heuristic for the traveling salesman problem: A comparison of meta-features. Neurocomputing, v. 205, p. 393 - 406, 2016. ISSN 0925-2312. Available: <https://doi.org/10.1016/j.neucom.2016.04.027>. Citations on pages 51 e 108.

KEUNG, J.; KOCAGUNELI, E.; MENZIES, T. Finding conclusion stability for selecting the best effort predictor in software effort estimation. Automated Software Engineering, v. 20, n. 4, p. 543-567, 2013. Available: <http://dx.doi.org/10.1007/ s10515-012-0108-5>. Citations on pages 49, 80, 90 e 100.

KIM, S.; WHITEHEAD JR., E. J.; ZHANG, Y. Classifying software changes: Clean or buggy? IEEE Trans. Softw. Eng., IEEE Press, v. 34, n. 2, p. 181-196, Mar. 2008. ISSN 0098-5589. Available: <http://dx.doi.org/10.1109/TSE.2007.70773>. Citation on page 40.

KITCHENHAM, B. A.; MENDES, E.; TRAVASSOS, G. H. Cross versus within-company cost estimation studies: A systematic review. IEEE Trans Soft Eng, v. 33, n. 5, p. 316-329, 2007. Available: <http://dx.doi.org/10.1109/TSE.2007.1001>. Citations on pages $26,45,48,73$ e 108 .

KOHAVI, R.; SOMMERFIELD, D. Feature subset selection using the wrapper method: Overfltting and dynamic search space topology. In: Proc. of the 1st Int. Conf. on 
Knowl. Disc. and Data Min. [S.1.]: AAAI Press, 1995. (KDD'95), p. 192-197. Citations on pages 99 e 101.

KONONENKO, I. Estimating attributes: Analysis and extensions of relief. In: Machine Learning: ECML-94. Springer Berlin Heidelberg, 1994. p. 171-182. Available: <http: //dx.doi.org/10.1007/3-540-57868-4_57>. Citations on pages 56, 58 e 61 .

KUNCHEVA, L. I. Combining Pattern Classifiers: Methods and Algorithms. [S.l.]: Wiley-Interscience, 2004. ISBN 0471210781. Citation on page 108.

LARSON, M. G. Descriptive statistics and graphical displays. Circulation, American Heart Association, Inc., v. 114, n. 1, p. 76-81, 2006. ISSN 0009-7322. Available: <https: //doi.org/10.1161/CIRCULATIONAHA.105.584474>. Citation on page 85.

LEE, T.; NAM, J.; HAN, D.; KIM, S.; IN, H. P. Micro interaction metrics for defect prediction. In: Proc. of the 19th ACM SIGSOFT Symp. and the 13th Eur. Conf. on Found. of Soft. Eng. ACM, 2011. (ESEC/FSE '11), p. 311-321. ISBN 978-1-4503-0443-6. Available: <http://dx.doi.org/10.1145/2025113.2025156>. Citation on page 40 .

LEMKE, C.; BUDKA, M.; GABRYS, B. Metalearning: a survey of trends and technologies. Artificial Intelligence Review, v. 44, n. 1, p. 117-130, 2015. Available: <http://dx.doi. org/10.1007/s10462-013-9406-y>. Citations on pages 27, 50, 51, 52 e 93.

LESSMANN, S.; BAESENS, B.; MUES, C.; PIETSCH, S. Benchmarking classification models for software defect prediction: A proposed framework and novel findings. IEEE Trans Soft Eng, v. 34, n. 4, p. 485-496, 2008. Available: <http://dx.doi.org/10.1109/ TSE.2008.35>. Citations on pages 26, 41, 44, 65 e 74.

LI, M.; ZHANG, H.; WU, R.; ZHOU, Z.-H. Sample-based software defect prediction with active and semi-supervised learning. Automated Software Engineering, Kluwer Academic Publishers, Hingham, MA, USA, v. 19, n. 2, p. 201-230, Jun. 2012. Available: $<$ http://dx.doi.org/10.1007/s10515-011-0092-1>. Citation on page 41.

LIAW, A.; WIENER, M. Classification and regression by randomforest. R News, v. 2, n. 3, p. 18-22, 2002. Available: <http://CRAN.R-project.org/doc/Rnews/>. Citation on page 65 .

LU, H.; CUKIC, B. An adaptive approach with active learning in software fault prediction. In: Proc. of the 8th Int. Conf. on Pred. Mod. in Soft. Eng. ACM, 2012. (PROMISE '12), p. 79-88. ISBN 978-1-4503-1241-7. Available: <http://dx.doi.org/10.1145/2365324. $2365335>$. Citation on page 41 .

LUACES, O.; DÍEZ, J.; BARRANQUERO, J.; COZ, J. J. del; BAHAMONDE, A. Binary relevance efficacy for multilabel classification. Prog. in Art. Intel., v. 1, n. 4, p. 303-313, 2012. Available: <http://dx.doi.org/10.1007/s13748-012-0030-x>. Citations on pages 53 e 97.

MA, Y.; LUO, G.; ZENG, X.; CHEN, A. Transfer learning for cross-company software defect prediction. Inf Soft Tech, Butterworth-Heinemann, v. 54, n. 3, p. 248-256, 2012. Available: <http://dx.doi.org/10.1016/j.infsof.2011.09.007>. Citations on pages 27, 47, $48,49,77,81,83$ e 87. 
MADEYSKI, L.; JURECZKO, M. Which process metrics can significantly improve defect prediction models? an empirical study. Software Quality Journal, Springer US, p. 1-30, 2014. ISSN 0963-9314. Available: <http://dx.doi.org/10.1007/s11219-014-9241-7>. Citations on pages 73, 74, 78, 79 e 108.

MAIMON, O; ROKACH, L. Data Mining and Knowledge Discovery Handbook. 2nd. ed. [S.l.]: Springer Publishing Company, Incorporated, 2010. ISBN 0387098224, 9780387098227. Citations on pages 42, 44, 49 e 53.

MALHOTRA, R. A systematic review of machine learning techniques for software fault prediction. Appl. Soft Comput., Elsevier Science Publishers B. V., Amsterdam, The Netherlands, v. 27, n. C, p. 504-518, Feb. 2015. Available: <http://dx.doi.org/10.1016/j. asoc.2014.11.023>. Citations on pages 35, 38, 42, 82, 83 e 98.

MANTOVAnI, R. G.; ROSSI, A. L. D.; VANSCHOREN, J.; BISCHL, B.; CARVALHO, A. C. P. L. F. To tune or not to tune: Recommending when to adjust svm hyper-parameters via meta-learning. In: Int. Joint Conf. on Neural Net. (IJCNN). [s.n.], 2015. p. 1-8. ISSN 2161-4393. Available: <http://dx.doi.org/10.1109/IJCNN.2015.7280644>. Citations on pages 51 e 93 .

MATHUR, A. P. Foundations of software testing. [S.l.]: Pearson Education, 2008. Citation on page 36 .

MCCABE, T. J. A complexity measure. IEEE Transactions on Software Engineering, SE-2, n. 4, p. 308-320, Dec 1976. ISSN 0098-5589. Available: <https://doi.org/10.1109/ TSE.1976.233837>. Citation on page 39.

MENZIES, T.; DEKHTYAR, A.; DISTEFANO, J.; GREENWALD, J. Problems with precision: A response to "comments on 'data mining static code attributes to learn defect predictors'". IEEE Trans Soft Eng, v. 33, n. 9, p. 637-640, 2007. Available: $<$ http://dx.doi.org/10.1109/TSE.2007.70721>. Citation on page 65.

MENZIES, T.; GREENWALD, J.; FRANK, A. Data mining static code attributes to learn defect predictors. IEEE Trans. on Soft. Eng., v. 33, n. 1, p. 2-13, Jan 2007. ISSN 0098-5589. Available: <http://dx.doi.org/10.1109/TSE.2007.256941>. Citations on pages 15 e 83.

MENZIES, T.; SHEPPERD, M. Special issue on repeatable results in software engineering prediction. Empirical Softw. Eng., v. 17, n. 1-2, p. 1-17, Feb. 2012. ISSN 1382-3256. Available: <http://dx.doi.org/10.1007/s10664-011-9193-5>. Citations on pages 29, 48, 49 e 78 .

MITCHELL, T. M. Machine Learning. 1. ed. [S.l.]: McGraw-Hill, Inc., 1997. ISBN 0070428077, 9780070428072. Citations on pages 27, 42 e 50.

MOCKUS, A.; VOTTA, L. G. Identifying reasons for software changes using historic databases. In: Proc. of the Int. Conf. on Soft. Maint. [S.l.]: IEEE Computer Society, 2000. (ICSM '00), p. 120-. ISBN 0-7695-0753-0. Citation on page 40.

MOSER, R.; PEDRYCZ, W.; SUCCI, G. A comparative analysis of the efficiency of change metrics and static code attributes for defect prediction. In: Proc Int Conf on Soft Eng (ICSE2008). [s.n.], 2008. p. 181-190. Available: <http://dx.doi.org/10.1145/1368088. $1368114>$. Citation on page 40. 
MUNSON, J. C.; KHOSHGOFTAAR, T. M. The detection of fault-prone programs. IEEE Trans. Softw. Eng., IEEE Press, v. 18, n. 5, p. 423-433, May 1992. ISSN 0098-5589. Available: <http://dx.doi.org/10.1109/32.135775>. Citations on pages 39 e 40.

MYERS, G. J.; SANDlER, C.; BADGETT, T. The Art of Software Testing. 3rd. ed. [S.1.]: Wiley Publishing, 2011. ISBN 1118031962, 9781118031964. Citations on pages 25, 35 e 37.

NAGAPPAN, N.; BALL, T. Use of relative code churn measures to predict system defect density. In: Proc. of the 27th Int. Conf. on Soft. Eng. ACM, 2005. (ICSE '05), p. 284-292. ISBN 1-58113-963-2. Available: <http://dx.doi.org/10.1145/1062455.1062514>. Citation on page 40.

NAGAPPAN, N.; MURPHY, B.; BASILI, V. The influence of organizational structure on software quality: An empirical case study. In: Proc Int Conf on Soft Eng (ICSE2008). [s.n.], 2008. p. 521-530. Available: <http://dx.doi.org/10.1145/1368088.1368160>. Citation on page 40 .

NAM, J. Software defect prediction on unlabeled datasets. Phd Thesis (PhD Thesis) - Hong Kong University of Science and Technology, 2015. Citations on pages 15, 38 e 39.

NAM, J.; PAN, S. J.; KIM, S. Transfer defect learning. In: Proc Int Conf on Soft Eng (ICSE2013). [s.n.], 2013. p. 382-391. Available: <http://dx.doi.org/10.1109/ICSE.2013. 6606584>. Citations on pages 47, 48, 49, 80 e 97.

OSTRAnD, T. J.; WEYUKER, E. J.; BELL, R. M. Where the bugs are. In: Proc Int Symp on Soft Test and Analy (ISSTA2004). ACM, 2004. p. 86-96. Available: <http://dx.doi.org/10.1145/1007512.1007524>. Citations on pages 26, 40 e 55.

. Predicting the location and number of faults in large software systems. IEEE Trans on Soft Eng, v. 31, n. 4, p. 340-355, 2005. Available: <http://dx.doi.org/10.1109/ TSE.2005.49>. Citations on pages 55 e 66.

PAN, S. J.; YANG, Q. A survey on transfer learning. IEEE Trans. on Knowl. and Data Eng., v. 22, n. 10, p. 1345-1359, Oct 2010. ISSN 1041-4347. Available: <http: //dx.doi.org/10.1109/TKDE.2009.191>. Citations on pages 15, 26, 46, 47 e 77.

PARMEZAN, A. R. S.; LEE, H. D.; WU, F. C. Metalearning for choosing feature selection algorithms in data mining: Proposal of a new framework. Expert Systems with Applications, Pergamon, Tarrytown, United States of America, v. 75, p. 1-24, Jun 2017. Available: <http://dx.doi.org/10.1016/j.eswa.2017.01.013>. Citations on pages 51, 52, 93 e 99.

PEREIRA, D. G.; AFONSO, A.; MEDEIROS, F. M. Overview of friedman's test and post-hoc analysis. Commun. in Stat. - Simul. and Comp., v. 44, n. 10, p. 2636-2653, 2015. Available: <http://dx.doi.org/10.1080/03610918.2014.931971>. Citations on pages 84,85 e 100.

PETERS, F.; MENZIES, T. Privacy and utility for defect prediction: Experiments with morph. In: 34th Int. Conf. on Soft. Eng. (ICSE). [s.n.], 2012. p. 189-199. ISSN 0270-5257. Available: <http://dx.doi.org/10.1109/ICSE.2012.6227194>. Citation on page 40 . 
PETERS, F.; MENZIES, T.; MARCUS, A. Better cross company defect prediction. In: Proc Work Conf on Mining Soft Rep (MSR2013). IEEE, 2013. p. 409-418. Available: <http://dx.doi.org/10.1109/MSR.2013.6624057>. Citations on pages 15, 29, $47,56,57,58,65,67,73$ e 85 .

PINZGER, M.; NAGAPPAN, N.; MURPHY, B. Can developer-module networks predict failures? In: Proc Int Symp on Found of Soft Eng (SIGSOFT/FSE2008). ACM, 2008. p. 2-12. Available: <http://dx.doi.org/10.1145/1453101.1453105>. Citations on pages 26 e 40 .

PLATT, J. C. Advances in kernel methods. In: . [S.1.]: MIT Press, 1999. chap. Fast Training of Support Vector Machines Using Sequential Minimal Optimization, p. 185-208. ISBN 0-262-19416-3. Citation on page 43.

PORTO, F.; SIMAO, A. Feature subset selection and instance filtering for cross-project defect prediction - classification and ranking. CLEI Electronic Journal, v. 19, n. 3, p. 4:1-4:17, 2016. Available: <http://dx.doi.org/10.19153/cleiej.19.3.4>. Citations on pages $29,56,85$ e 107.

. Feature subset selection for instance filtering methods on cross-project defect prediction. In: Proc Ibero-American Conf on Soft Eng (CIBSE/ESELAW2016). Quito: [s.n.], 2016. p. 171-184. Citations on pages 29, 56 e 107.

PRATEEK, S.; PASALA, A.; ARACENA, L. M. Evaluating performance of network metrics for bug prediction in software. In: Asia-Pacific Soft Eng Conf (APSEC2013). [s.n.], 2013. v. 1, p. 124-131. Available: <http://dx.doi.org/10.1109/APSEC.2013.27>. Citations on pages 60 e 74 .

PRESSMAN, R. S. Software Engineering: A Practitioner's Approach. 7. ed. [S.l.]: McGraw-Hill Science, 2009. Citations on pages 25, 34, 35, 36 e 37.

RAHMAN, F.; POSNETT, D.; HINDLE, A.; BARR, E.; DEVANBU, P. Bugcache for inspections: Hit or miss? In: Proc. of the 19th ACM SIGSOFT Symp. and the 13th Eur. Conf. on Found. of Soft. Eng. ACM, 2011. (ESEC/FSE '11), p. 322-331. ISBN 978-1-4503-0443-6. Available: <http://dx.doi.org/10.1145/2025113.2025157>. Citation on page 40 .

RAMAMOORTHY, C.; BASTANI, F. Software reliability - status and perspectives. v. 8, p. 354-371, 1982. Citation on page 37.

RAPPS, S.; WEYUKER, E. J. Selecting software test data using data flow information. IEEE Trans. Softw. Eng., v. 11, p. 367-375, 1985. Citation on page 36.

REIF, M.; SHAFAIT, F.; GOLDSTEIN, M.; BREUEL, T.; DENGEL, A. Automatic classifier selection for non-experts. Pattern Analysis and Applications, v. 17, n. 1, p. 83-96, 2014. Available: <http://dx.doi.org/10.1007/s10044-012-0280-z>. Citation on page 51.

RICE, J. R. The algorithm selection problem. Advances in Computers, v. 15, p. 65 - 118, 1976. ISSN 0065-2458. Available: <http://dx.doi.org/10.1016/S0065-2458(08)60520-3>. Citation on page 50. 
ROMANSKI, P.; KOTTHOFF, L. FSelector: Selecting attributes. [S.l.], 2014. R package version 0.20. Available: <http://CRAN.R-project.org/package=FSelector $>$. Citation on page 63 .

ROUSSEEUW, P. Silhouettes: A graphical aid to the interpretation and validation of cluster analysis. J. Comput. Appl. Math., Elsevier Science Publishers B. V., Amsterdam, The Netherlands, The Netherlands, v. 20, n. 1, p. 53-65, Nov. 1987. Available: <http: //dx.doi.org/10.1016/0377-0427(87)90125-7>. Citation on page 97.

SANTOS, D. P. dos; CARVAlHO, A. C. P. L. F. de. Automatic selection of learning bias for active sampling. In: Brazilian Conf. on Intel. Syst. (BRACIS2016). [s.n.], 2016. p. 55-60. Available: <https://dx.doi.org/10.1109/BRACIS.2016.021>. Citations on pages $18,51,96,97$ e 98 .

SCHAPIRE, R. E.; SINGER, Y. Boostexter: A boosting-based system for text categorization. Machine Learning, v. 39, n. 2, p. 135-168, 2000. Available: <http: //dx.doi.org/10.1023/A:1007649029923>. Citations on pages 53 e 98.

SCHOLKOPF, B.; SMOLA, A. J. Learning with Kernels: Support Vector Machines, Regularization, Optimization, and Beyond. [S.l.]: MIT Press, 2001. ISBN 0262194759. Citation on page 42.

SERBAN, F.; VANSCHOREN, J.; KIETZ, J.-U.; BERNSTEIN, A. A survey of intelligent assistants for data analysis. ACM Comput. Surv., ACM, v. 45, n. 3, p. 31:1-31:35, Jul. 2013. ISSN 0360-0300. Available: <https://doi.org/10.1145/2480741.2480748>. Citations on pages 15 e 50 .

SHEN, V. Y.; YU, T.-J.; THEBAUT, S. M.; PAULSEN, L. R. Identifying error-prone software an empirical study. IEEE Trans. Softw. Eng., IEEE Press, v. 11, n. 4, p. 317-324, Apr. 1985. ISSN 0098-5589. Available: <http://dx.doi.org/10.1109/TSE.1985. $232222>$. Citations on pages 39 e 40.

SHEPPERD, M.; BOWES, D.; HALL, T. Researcher bias: The use of machine learning in software defect prediction. IEEE Trans. on Soft. Eng., v. 40, n. 6, p. 603-616, June 2014. ISSN 0098-5589. Available: <https://doi.org/10.1109/TSE.2014.2322358>. Citations on pages 29, 48 e 78 .

SIMAO, A. S. The application of mutation testing in the context of testing and validation of coloured Petri nets. Phd Thesis (PhD Thesis) - Instituto de Ciências Matemáticas e de Computação, Universidade de São Paulo, São Carlos, 2004. Available: <http://dx.doi.org/10.11606/T.55.2004.tde-13042005-152434>. Citation on page 37.

SOMMERVILLE, I. Software Engineering. 10th. ed. [S.l.]: Pearson, 2015. ISBN 0133943038, 9780133943030. Citation on page 33.

SONG, L.; MINKU, L. L.; YAO, X. The impact of parameter tuning on software effort estimation using learning machines. In: Proc. of the 9th Int. Conf. on Pred. Mod. in Soft. Eng. [s.n.], 2013. (PROMISE '13), p. 9:1-9:10. ISBN 978-1-4503-2016-0. Available: $<$ http://dx.doi.org/10.1145/2499393.2499394>. Citations on pages 29 e 78.

TABA, S. E. S.; KHOMH, F.; ZOU, Y.; HASSAN, A. E.; NAGAPPAN, M. Predicting bugs using antipatterns. In: Proc IEEE Int Conf on Soft Maint (ICSM2013). [s.n.], 
2013. p. 270-279. Available: <http://dx.doi.org/10.1109/ICSM.2013.38>. Citation on page 40 .

TAN, M.; TAN, L.; DARA, S.; MAYEUX, C. Online defect prediction for imbalanced data. In: 2015 IEEE/ACM 37th IEEE Int. Conf. on Soft. Eng. [s.n.], 2015. v. 2, p. 99-108. ISSN 0270-5257. Available: <http://dx.doi.org/10.1109/ICSE.2015.139>. Citation on page 40 .

TANTITHAMTHAVORN, C.; MCINTOSH, S.; HASSAN, A. E.; MATSUMOTO, K. Comments on "researcher bias: The use of machine learning in software defect prediction". IEEE Transactions on Software Engineering, v. 42, n. 11, p. 1092-1094, Nov 2016. ISSN 0098-5589. Available: <http://dx.doi.org/10.1109/TSE.2016.2553030>. Citations on pages $29,48,49$ e 78 .

TORGO, L. Data Mining with R, learning with case studies. [S.l.]: Chapman and Hall/CRC, 2010. ISBN 1439810184, 9781439810187. Citation on page 63.

TOSUN, A.; TURHAN, B.; BENER, A. Validation of network measures as indicators of defective modules in software systems. In: Proc Int Conf on Pred Mod in Soft Eng (PROMISE2009). ACM, 2009. p. 5:1-5:9. Available: <http://dx.doi.org/10.1145/ $1540438.1540446>$. Citation on page 60 .

TSOUMAKAS, G.; KATAKIS, I. Multi-label classification: An overview. IJDWM, v. 3, n. 3, p. 1-13, 2007. Available: <http://dx.doi.org/10.4018/jdwm.2007070101>. Citations on pages 52 e 93 .

TURHAN, B.; MENZIES, T.; BENER, A. B.; STEFANO, J. D. On the relative value of cross-company and within-company data for defect prediction. Emp Soft Eng, Kluwer Academic Publishers, v. 14, n. 5, p. 540-578, 2009. Available: <http://dx.doi.org/10.1007/ s10664-008-9103-7>. Citations on pages 26, 27, 29, 47, 49, 56, 57, 58, 65, 73, 77 e 80.

VILALTA, R.; DRISSI, Y. A perspective view and survey of meta-learning. Artificial Intelligence Review, v. 18, n. 2, p. 77-95, Jun 2002. Available: <https://doi.org/10. 1023/A:1019956318069>. Citations on pages 28 e 50.

WATANABE, S.; KAIYA, H.; KAIJIRI, K. Adapting a fault prediction model to allow inter language reuse. In: Proc Int Conf on Pred Mod in Soft Eng (PROMISE2008). ACM, 2008. p. 19-24. Available: <http://dx.doi.org/10.1145/1370788.1370794>. Citations on pages $27,47,49,77$ e 80 .

WEYUKER, E. J.; OSTRAND, T. J.; BELL, R. M. Comparing the effectiveness of several modeling methods for fault prediction. Emp Softw Eng, v. 15, p. 277-295, 2010. Available: <http://dx.doi.org/10.1007/s10664-009-9111-2>. Citations on pages 38, 40, $55,65,66$ e 74 .

WITTEN, D. M.; TIBSHIRANI, R. A framework for feature selection in clustering. Jour Amer Statist Assoc, v. 105, n. 490, p. 713-726, 2010. Available: <http://dx.doi.org/10. 1198/jasa.2010.tm09415>. Citations on pages 56, 58, 61 e 63.

WOLPERT, D. H. The supervised learning no-free-lunch theorems. In: Soft Comp. and Indus.: Recent Applications. London: Springer London, 2002. p. 25-42. ISBN 978-1-4471-0123-9. Available: <http://dx.doi.org/10.1007/978-1-4471-0123-9_3>. Citations on pages 27, 48 e 93. 
WONG, W. E.; MATHUR, A. P. Fault detection effectiveness of mutation and data flow testing. Software Quality Journal, Springer Netherlands, v. 4, p. 69-83, 1995. Citation on page 37 .

WU, X.; ZHOU, Z. A unified view of multi-label performance measures. CoRR, abs/1609.00288, 2016. Available: <http://arxiv.org/abs/1609.00288>. Citation on page 98.

XU, R.; WUNSCH, D. Clustering. [S.l.]: Wiley-IEEE Press, 2009. ISBN 9780470276808. Citation on page 97.

YANG, X.; TANG, K.; YAO, X. A learning-to-rank approach to software defect prediction. IEEE Trans on Reliability, v. 64, p. 234-246, 2015. Available: <http://dx.doi.org/10. 1109/TR.2014.2370891>. Citations on pages 38, 40, 55, 65, 66, 69, 73, 74 e 108.

ZHANG, F.; MOCKUS, A.; KEIVANLOO, I.; ZOU, Y. Towards building a universal defect prediction model. In: Proc Work Conf on Mining Soft Rep (MSR2014). ACM, 2014. p. 182-191. Available: <http://dx.doi.org/10.1145/2597073.2597078>. Citations on pages 47, 74 e 108 .

ZHANG, F.; ZHENG, Q.; ZOU, Y.; HASSAN, A. E. Cross-project defect prediction using a connectivity-based unsupervised classifier. In: Proc Int Conf on Soft Eng (ICSE2016). New York, NY, USA: ACM, 2016. p. 309-320. Available: <http://dx.doi.org/10.1145/ 2884781.2884839>. Citation on page 74 .

ZHANG, M. L.; ZHOU, Z. H. A review on multi-label learning algorithms. IEEE Trans. on Knowl. and Data Eng., v. 26, n. 8, p. 1819-1837, Aug 2014. ISSN 1041-4347. Available: <http://dx.doi.org/10.1109/TKDE.2013.39>. Citation on page 97.

ZHAO, Z.; MORSTATTER, F.; SHARMA, S.; ALELYANI, S.; ANAND, A.; LIU, H. Advancing feature selection research - ASU feature selection repository. [S.l.], 2011. Citations on pages 56, 58, 61 e 63 .

ZIMMERMANN, T.; NAGAPPAN, N. Predicting defects using network analysis on dependency graphs. In: Proc Int Conf on Soft Eng (ICSE2008). [s.n.], 2008. p. 531-540. Available: <http://dx.doi.org/10.1145/1368088.1368161>. Citations on pages $17,26,40,55,56,60,63,65$ e 73.

ZIMMERMANN, T.; NAGAPPAN, N.; GALL, H.; GIGER, E.; MURPHY, B. Cross-project defect prediction: A large scale experiment on data vs. domain vs. process. In: Proc Int Symp on Found of Soft Eng (SIGSOFT/FSE2009). ACM, 2009. p. 91-100. Available: <http://dx.doi.org/10.1145/1595696.1595713>. Citations on pages 26, 46, 49, 67 e 85.

ZIMMERMAnN, T.; PREMRAJ, R.; ZELLER, A. Predicting defects for eclipse. In: Proc Int Conf on Pred Mod in Soft Eng (PROMISE2007). IEEE, 2007. p. 9-. Available: <http://dx.doi.org/10.1109/PROMISE.2007.10>. Citations on pages 74 e 108. 
APPENDIX

A

\section{GENERAL AUC PERFORMANCES}

Table 20 - Part I - General AUC performances for all the evaluated CPDP methods (Base and Meta). The methods are ordered by the mean AUC value.

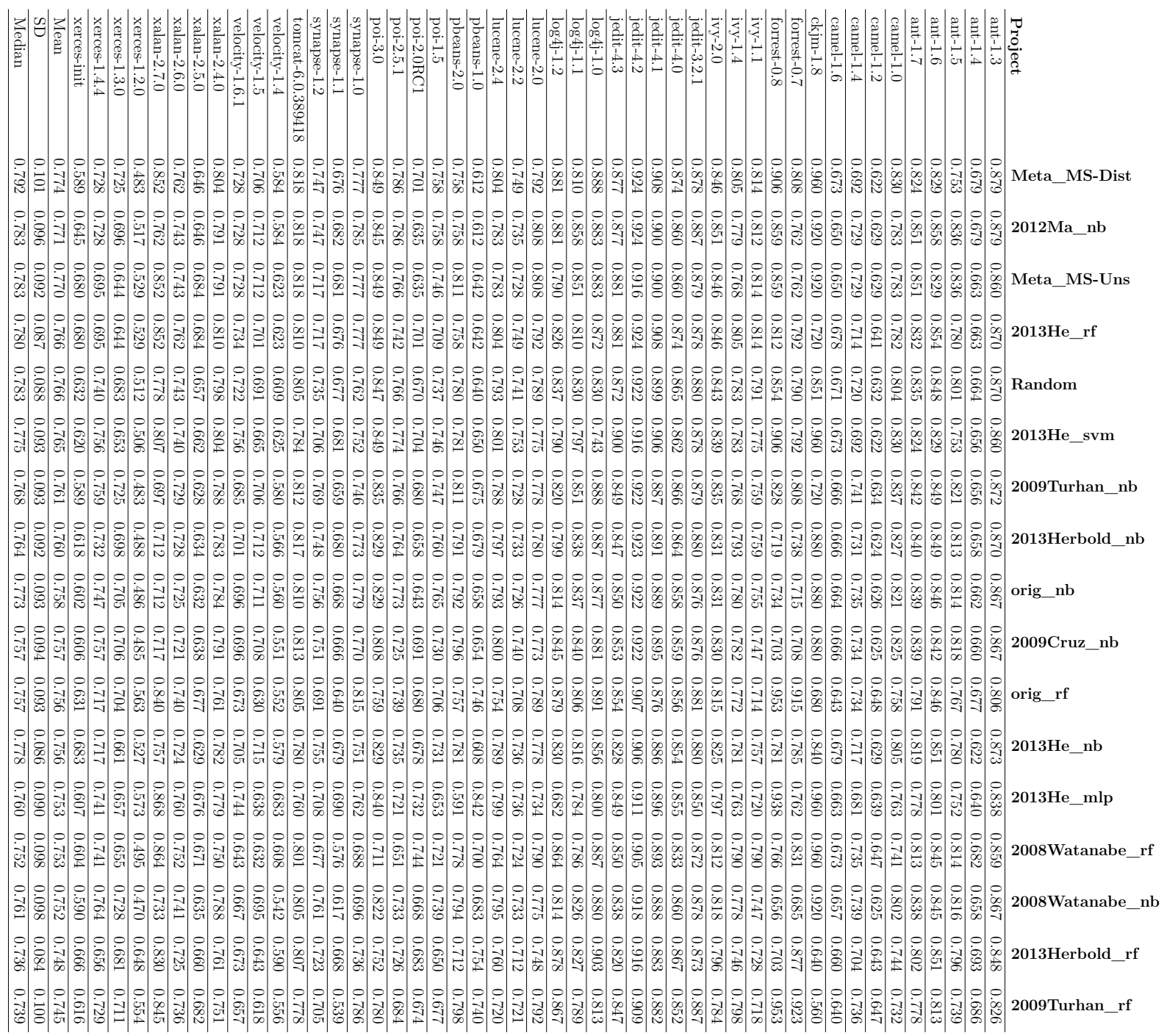


Table 21 - Part II - General AUC performances for all the evaluated CPDP methods (Base and Meta). The methods are ordered by the mean AUC value.

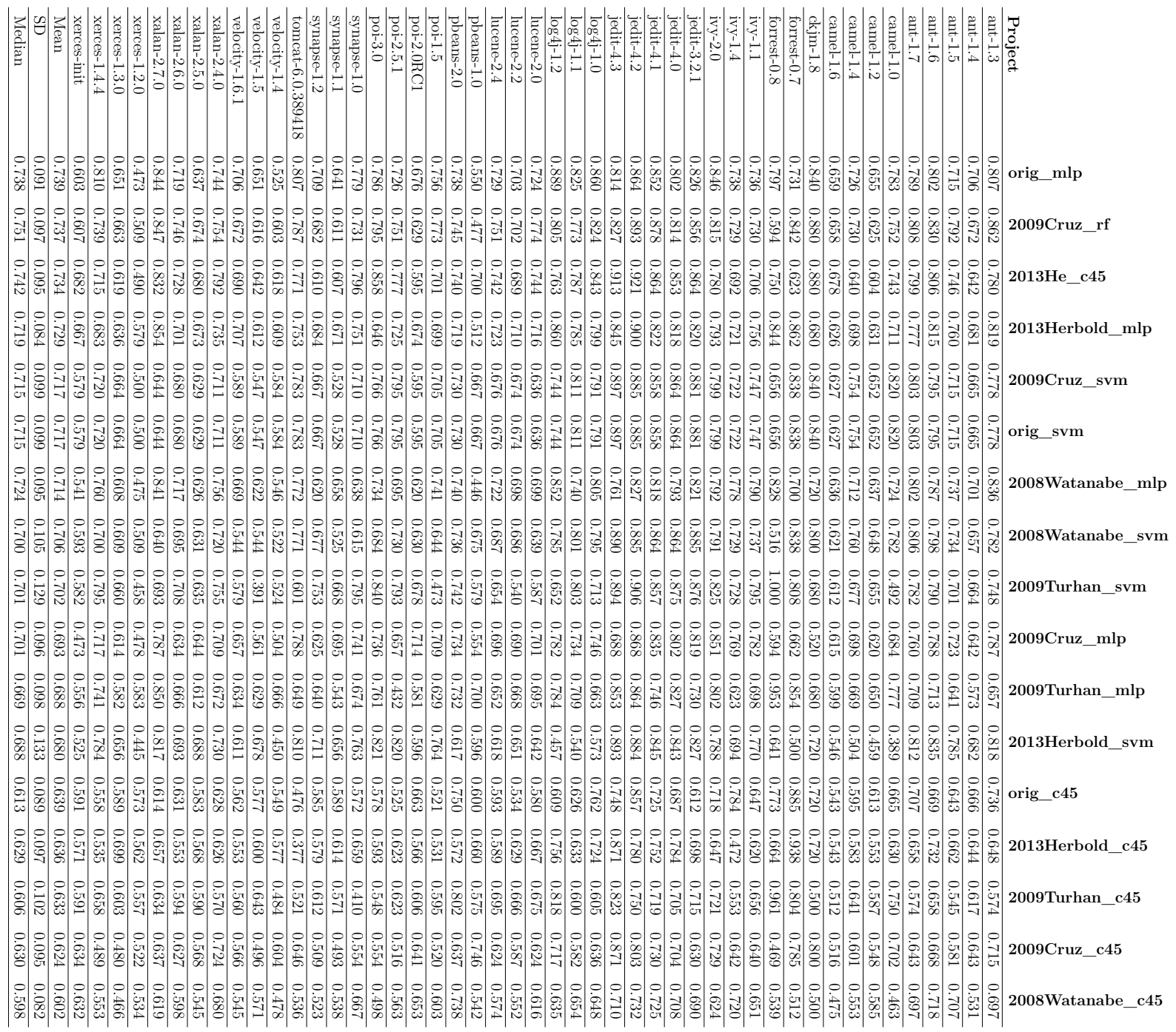




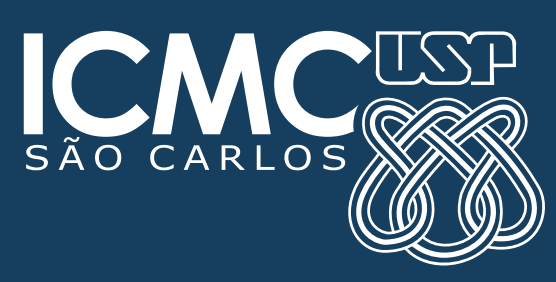

